\title{
THE INFLUENCE OF LIFTING CONDENSATION LEVEL ON LOW- LEVEL OUTFLOW AND ROTATION IN SIMULATED SUPERCELL \\ THUNDERSTORMS
}

\author{
A Thesis \\ by \\ MATTHEW BROWN \\ Submitted to the Office of Graduate and Professional Studies of \\ Texas A\&M University \\ in partial fulfillment of the requirement for the degree of \\ MASTER OF SCIENCE
}

\begin{abstract}
Chair of Committee, Christopher Nowotarski
Committee Members, Craig Epifanio

Robert Hetland
\end{abstract}

Head of Department, Ping Yang

August 2018

Major Subject: Atmospheric Sciences

Copyright 2018 Matthew Brown 


\begin{abstract}
Supercell thunderstorms are simulated using a numerical model in order to probe the effects of changing low-level moisture, and thus lifting condensation level (LCL), on outflow characteristics and the evolution of near-surface rotation. A set of three thermodynamic profiles corresponding to different LCLs are tested with varying low-level shear orientations.
\end{abstract}

The thermodynamic properties of the simulations are sensitive to variations in LCL, with higher LCLs contributing to broader, more negatively buoyant cold pools. These outflow characteristics exhibit a distinct influence on the positioning of near-surface rotation relative to the mid-level mesocyclone. As LCL increases and outflow becomes more negatively buoyant, there is more forward propagation of near-surface circulation relative to the mesocyclone aloft. It is shown that there is a negative, statistically significant lag correlation between the separation of these mid- and low-level circulations and subsequent production of near-surface vertical vorticity, suggesting that the collocation of these circulations affects the convergence and stretching of circulation-rich air near the surface. The degree to which the overlap of mid- and low-level circulations intensifies preexisting near-surface vertical vorticity depends on a number of interrelated factors, including the low-level storm-relative wind profile and the availability of surface rotation for stretching. That said, these simulations suggest that such an alignment is a necessary prerequisite for the strengthening of near-surface vertical vorticity to occur. Thus, for a given low-level shear orientation, LCL can influence whether appreciable near-surface vertical vorticity is able to form within the storm. 


\section{ACKNOWLEDGEMENTS}

This study was funded by NSF grant AGS-1446342. Thank for all the feedback and advice from

all of the members of my thesis committee - Christopher Nowotarski, Craig Epifanio, and

Robert Hetland - especially Chris for enduring constant questions and late night emails to help me propel this project forward to where it is now. Lastly, an acknowledgement must be given to George Bryan for the development and maintaining of his brilliant CM1 model, and for making it available to everyone in our field to utilize. 


\section{TABLE OF CONTENTS}

Page

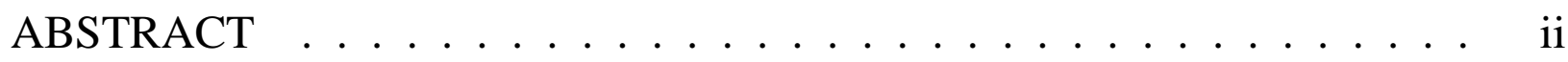

ACKNOWLEDGEMENTS ................. iii

TABLE OF CONTENTS . . . . . . . . . . . . . . . . iv

CONTRIBUTORS AND FUNDING SOURCES $\ldots \ldots \ldots$. . . . . . vi

LIST OF FIGURES . . . . . . . . . . . . . . . . . . vii

LIST OF TABLES . . . . . . . . . . . . . . . . . . xiii

1 INTRODUCTION $\ldots \ldots \ldots \ldots \ldots \ldots$

2 BACKGROUND ....................... 4

$2.1 \quad$ Supercell Structure $\ldots \ldots \ldots \ldots$

2.2 Origins of Rotation within the Supercell _. . . . . . . . . 6

2.2 .1 Mid-level Rotation . . . . . . . . . . . . . . . 6

2.2 .2 Near-Surface Rotation . . . . . . . . . . . . . . 8

2.3 Influences of Low-Level Shear and LCL Height $\ldots \ldots 13$

2.4 Motivation and Research Questions _ . . . . . . . . . . . . 17

3 DATA AND METHODS . . . . . . . . . . . . . . . . 19

3.1 Model Configuration . . . . . . . . . . . . . . . . . 19

3.2 Model Initialization $\ldots \ldots \ldots 21$

3.2 .1 Wind Profile . . . . . . . . . . . . . . 22 
3.2.2 Thermodynamic Profile . . . . . . . . . . . 23

4 RESULTS AND DISCUSSION . . . . . . . . . . . . 26

4.1 Thermodynamic Characteristics of Outflow as a Function of LCL _ . . 26

4.2 Effect of LCL on Storm Structure and Circulation _ . . . . . . . 33

4.3 Effect of Circulation Overlap on Low-Level Vorticity Production . . . . . 41

4.3.1 Reflectivity and Circulation Structure _. . . . . . . . 41

4.3.2 Time Series Analysis _ . . . . . . . . . . . . . 45

4.3.3 Distributions of Derived Variables ～. . . . . . . . . . 48

4.3.4 Statistical Analysis . . . . . . . . . . . . . . 52

4.4 Dynamic Influences of LCL on Vorticity Generation $\ldots \ldots$

4.5 Additional Considerations . . . . . . . . . . . . . 62

$5 \quad$ SUMMARY AND CONCLUSIONS $\ldots \ldots \ldots 6$

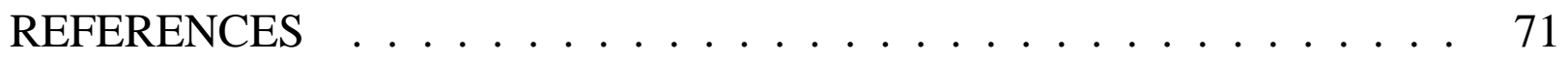

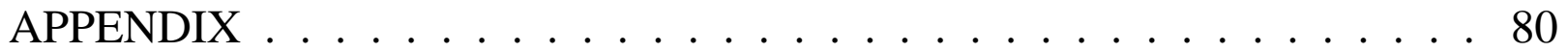




\section{CONTRIBUTORS AND FUNDING SOURCES}

\section{Contributors}

This work was supported by a thesis committee consisting of Professor Christopher Nowotarski [advisor] and Professor Craig Epifanio of the Department of Atmospheric Sciences and Professor Robert Hetland of the Department of Oceanography. All work conducted for the thesis was completed by the student independently.

\section{Funding Sources}

This work was made possible in part by the National Science Foundation (NSF) under grant AGS-1446342. Its contents are solely the responsibility of the authors and do not necessarily represent the official views of NSF. 


\section{LIST OF FIGURES}

FIGURE

Page

$1 \quad$ Plan view of a tornadic supercell, including the updraft (UD), forward flank downdraft (FFD), and rear flank downdraft (RFD) [adapted from Lemon and Doswell (1979)] . . . . . . . . . . . . . . . . . . .

2 3-D kinematic diagram of a tornadic supercell (NOAA National Severe Storms Laboratory 2018) . . . . . . . . . . . . . . . . . . 5

3 Simplified time evolution of vorticity streamlines in the presence of an updraft (Markowski and Richardson 2009, Fig. 2a-c) . . . . . . . . . . . . . . . . . 7

4 Diagram showing contributions of environmental shear (left side) and baroclinic overturning (right side) to vertical vorticity production in a tornadic supercell (Markowski 2018) . . . . . . . . . . . . . . . . 9

5 Simplified time evolution of vorticity streamline adjustment in the presence of a downdraft (Markowski and Richardson 2009, Fig. 2d-e) . . . . . . . . 10

6 (a) Baroclinically generated vortex rings encircling a buoyancy minimum that extends throughout a vertical column (such a region of negative buoyancy might be found in the hook echo/RFD region of a supercell, for example (b) Vortex rings being swept forward and tilted upward on their downstream sides as they descend toward the ground owing to the additional presence of rear-to-front flow through the buoyancy minimum (c) The leading edges of the vortex rings are lifted by an updraft in close proximity to the buoyancy minimum, leading to arching vortex 
(Figure 6, continued) lines and a couplet of cyclonic ("C") and anticyclonic ("A") vertical vorticity that straddles the buoyancy minimum and associated downdraft (Markowski et al. 2008, Fig. 17) . . . . . . . . . . . . . . . 11

7 Diagram of the orientation of a buoyant updraft (a) without the presence of a cold pool or environmental shear, (b) in the presence of a cold pool and a unidirectional shear profile, (c) with no cold pool and an easterly shear profile, and (d) in the presence of both a cold pool and an easterly shear profile [adapted from Fig. 5 in Rotunno et al. (1988)] . . . . . . . . . . . . . . 14

8 Numerical simulations of an eastward-moving density current in an environment containing (a) westerly wind shear, (b) no wind shear, and (c) easterly wind shear, with potential temperature perturbations contoured at $1 \mathrm{~K}$ intervals within the cold pool, starting at $-1 \mathrm{~K}$; wind vectors are relative to the density current $(\mathrm{Uc} \approx 19,11$, and $8 \mathrm{~m} \mathrm{~s}^{-1}$ in the easterly shear, no shear, and westerly shear cases, respectively), and the ground-relative vertical wind profiles are shown to the right of each case (Markowski and Richardson 2011, Fig. 5.31) _ . . . . . . . . 15

9 Schematic of model hodograph, with low-level alterations displayed [adapted from Fig. 2 in Guarriello et al. (2018)] . . . . . . . . . . . . . 22

10 Sample thermodynamic soundings used for model initialization (with the $\alpha=0^{\circ}$ wind profile shown arbitrarily) $\ldots \ldots \ldots$. . . . . . . . . . 24 
11 Hourly plots of near-surface buoyancy $\left(\mathrm{m} \mathrm{s}^{-2}\right)$ for the $\alpha=0^{\circ}$ case, with contours of accumulated precipitation translated with the moving domain in black plotted every $0.25 \mathrm{~cm}$; the black dot is the $2-5 \mathrm{~km}$ integrated updraft helicity maximum, and the blue dotted line is the contour of $-1 \mathrm{~K}$ potential temperature perturbation

12 Same as Figure 11, but for the $\alpha=90^{\circ}$ case . . . . . . . . . . . . 28

13 Time series of average cold pool buoyancy within the inner domain for each LCL across all low level shear orientations, as well as the $\alpha=0^{\circ}$ and $\alpha=90^{\circ}$ cases . . . 29

14 Box and whisker plots of the fraction of gridpoints in the cold pool (i.e., gridpoints with potential temperature perturbations of $-1 \mathrm{~K}$ or less), accumulated precipitation translated with the moving domain $(\mathrm{cm})$, and buoyancy within the cold pool $\left(\mathrm{m} \mathrm{s}^{-2}\right)$ within the inner domain during the last 90 minutes of model integration for all shear orientations, as well as the $\alpha=0^{\circ}$ and $\alpha=90^{\circ}$ cases. . . . . . . . . . 31

15 Relative humidity profiles within the lowest $5 \mathrm{~km}$ of each thermodynamic sounding, with dotted lines marking the approximate LCL height in each sounding . . . . . 33

16 Hourly near-surface circulation for the $\alpha=0^{\circ}$ simulations; the dashed blue line is the $-1 \mathrm{~K}$ potential temperature contour at the lowest model grid level, black contours are mid-level (4 km AGL) vertical velocity plotted every $5 \mathrm{~m} \mathrm{~s}^{-1}$, the solid magenta line is the $500 \mathrm{~m}^{2} \mathrm{~s}^{-2} 2-5 \mathrm{~km}$ integrated updraft helicity contour, the black dot is the maximum updraft helicity, and the green marks the location of the maximum near-ground circulation within the inner domain introduced earlier (outlined in black); the units of the $\mathrm{x}$ and $\mathrm{y}$ axes are kilometers. . . . . . . . . . . . . 34 
17 Same as Figure 16, but for the $\alpha=90^{\circ}$ simulations $\ldots \ldots 36$

18 Time series of the mesocyclone separation distance (blue), the maximum vertical vorticity at the lowest grid level (red), and the maximum integrated updraft helicity (green) for all LCLs and low level shear orientations; the dashed black line represents the zero-line for mesocyclone separation distance $\ldots \ldots \ldots 38$

19 Reflectivity plots of storm simulations shown at the time of maximum vertical vorticity at the lowest grid level; the dashed blue line is the $-1 \mathrm{~K}$ potential temperature contour at the lowest model grid level, black contours are mid-level (4 $\mathrm{km}$ ) vertical velocity plotted every $5 \mathrm{~m} \mathrm{~s}^{-1}$, the solid magenta line is the $500 \mathrm{~m}^{2} \mathrm{~s}^{-2}$ $2-5 \mathrm{~km}$ integrated updraft helicity contour, the black dot is the maximum updraft helicity, and the yellow star marks the location of the maximum near-ground (surface) vertical vorticity; the units of the $\mathrm{x}$ and $\mathrm{y}$ axes are kilometers. . . . . . 42

20 Surface circulation for the same simulations and time steps shown in Figure 16, with the same vertical vorticity and updraft helicity contours, and updraft helicity maxima overlaid; the wind vectors represent storm-relative flow, the black box represents the inner domain in which mesocyclone separation distance is calculated, with the green diamond representing the maximum circulation within said box. . . . . . . . . . . . . . . . . . . . 44

21 Box and whisker plots of mesocyclone separation distance, maximum vertical vorticity at the lowest grid level, average circulation under the mid-level mesocyclone (i.e., circulation beneath the $500 \mathrm{~m} 2 \mathrm{~s}-2$ updraft helicity contour shown in earlier figures), and circulation fraction as a function of LCL for all low-level shear orientations _ . . . . . . . . . . . . . . . . 49 
22 Same as Figure 21, but for the $\alpha=0^{\circ}$ simulations . . . . . . . . . . . 51

23 Same as Figure 21, but for the $\alpha=90^{\circ}$ simulations . . . . . . . . . . . 52

24 Scatterplot of maximum near-surface vertical vorticity (taken at 5-minute interval over the last two hours of each simulation) as a function of the magnitude of mesocyclone separation distance. The ordinate is lagged by 5 minutes from the abscissa. The linear regression line for each LCL is shown, and the legend contains correlation coefficient $(\mathrm{R})$ of each relationship, followed by its associated p-value. 54

25 Time series of the average near-surface circulation (top) and fraction of gridpoints with near-surface circulation exceeding $5000 \mathrm{~m}^{2} \mathrm{~s}^{-1}$ beneath the low-level mesocyclone (bottom), defined as the area at $\mathrm{z}=1 \mathrm{~km}$ where vertical velocity is greater than $1 \mathrm{~m} \mathrm{~s}^{-1}$ and circulation exceeds $10000 \mathrm{~m}^{2} \mathrm{~s}^{-1}$. . . . . . . . 55

26 Same as Figure 24 (including 5-minute lag of the ordinate), but for circulation fraction as defined in Figure 25, and maximum near-surface vertical vorticity . . 56

27 Plan views of dynamic vertical perturbation pressure gradient force $500 \mathrm{~m}$ AGL (shaded), circulation at the lower model surface (black contours of $0.5 \times 10^{3}$, $1 \times 10^{4}, 2 \times 10^{4}, 3 \times 10^{4}, 4 \times 10^{4}, 5 \times 10^{4} \mathrm{~m}^{2} \mathrm{~s}^{-1}$ ), storm-relative surface winds, and the $-1 \mathrm{~K}$ surface potential temperature perturbation (dashed blue) for the $\alpha=0^{\circ}$ and $\alpha=90^{\circ}$ cases five minutes (one time step) before maximum near-surface vertical vorticity. The solid magenta line is the $500 \mathrm{~m}^{2} \mathrm{~s}^{-2}$ updraft helicity contour, and the black dot is the maximum updraft helicity. . . . . . . . . 60 
28 Hourly reflectivity fields for the storm simulations initialized with varying surface pressures of 950,1000 , and $1050 \mathrm{mb}$. . . . . . . . . .

29 Hourly circulation fields for the storm simulations initialized with varying surface pressures of 950,1000 , and $1050 \mathrm{mb}$. . . . . . . . . . .

30 Time series comparison of maximum near-surface vertical vorticity, maximum integrated updraft helicity, maximum updraft (at 4 km AGL), mesocyclone separation distance, average near-surface circulation within the inner window defined earlier, and fraction of gridpoints with near-surface circulation exceeding $5,000 \mathrm{~m}^{2} / \mathrm{s}$ beneath the low-level mesocyclone (i.e., the area at $\mathrm{z}=1$ $\mathrm{km}$ where vertical velocity is greater than $1 \mathrm{~m} \mathrm{~s}^{-1}$ and circulation exceeds 10,000 $\mathrm{m}^{2} \mathrm{~s}^{-1}$ ) for the three surface pressure simulations from Figure $28 . \quad$. . . . . . 86

$312-\mathrm{D}$ numerical simulations of a eastward-propagating cold pool with the $\alpha=0^{\circ}$ low-level shear profile and varying surface pressures $\left(\mathrm{p}_{\mathrm{s}}\right)$ of 950, 1000, and 1050 $\mathrm{mb}$, shown at $3600 \mathrm{~s}$ into the simulation. The outline of the cold pool is marked in blue by negative theta perturbations contoured at $1 \mathrm{~K}$ intervals (starting with $-1 \mathrm{~K}$ ); the wind vectors plotted are relative to the propagation speed of the cold pool $(\mathrm{Uc} \approx 15 \mathrm{~m} / \mathrm{s})$. . . . . . . . . . . . . . . . . . . . . . 88

32 Same as Figure 27, but for the $\alpha=90^{\circ}$ case (with $U c \approx 12.5 \mathrm{~m} \mathrm{~s}^{-1}$ ) . . . . . . 89 


\section{LIST OF TABLES}

TABLE

Page

Table of model specifications for CM1 simulations . . . . . . . . . . . . .

2 Tables of surface (SFC), mixed layer (ML), and most unstable (MU) CAPE;

SFC/ML/MU convective inhibition (CIN)F [all with units of $\mathrm{J} \mathrm{kg}^{-1}$ ]; SFC LCL, level of free convection (LFC), and equilibrium level (EL) [all with units of meters] in the three thermodynamic soundings . . . . . . . . . . 25

3 Simulation names by LCL height and shear vector orientation angle $(\alpha)$. . . . 26

4 Average gust-front orientation angle (relative to a N-S orientation, with north corresponding to $0^{\circ}$ and east to $90^{\circ}$ ), and the component of $0-1 \mathrm{~km}$ storm-relative winds opposing the gust front . . . . . . . . . . . . . . . . 40

5 Maximum vertical vorticity at the lowest grid level for each of the model configurations (with highest vorticity for each shear orientation shown in red, and the lowest in blue) . . . . . . . . . . . . . . . . . 47

6 Average circulation $\left(\mathrm{m}^{2} \mathrm{~s}^{-1}\right)$ and dynamic VPPGF $\left(\mathrm{m} \mathrm{s}^{-2}\right)$ beneath the mid-level mesocyclone (i.e., the $500 \mathrm{~m}^{2} \mathrm{~s}^{-2}$ updraft helicity contour) at the model time steps shown in Figure 23 . . . . . . . . . . . . . . . . . . . 61 
7 Same as Table 2, but for the three Weisman-Klemp pressure soundings . . . . . 79

8 Table of model specifications for cold pool simulations . . . . . . . . . . 81 


\section{INTRODUCTION}

The supercell thunderstorm poses a unique scientific challenge due to its tangible societal and economic impacts paired with the physical complexity clouding our understanding of its structure and evolution. Characterized by the presence of a sustained, deep rotating updraft referred to as a mesocyclone, supercell thunderstorms produce a number of hazards including heavy precipitation and flash flooding, intense winds, and hail. Perhaps their most well-known feature, however, is their ability to produce tornadoes (Lemon and Doswell 1979; Weisman and Klemp 1986, Brooks et al. 1994; Thompson et al. 2003, Coffer and Parker 2015). Though not all tornadoes form within supercells, the majority of significant and violent tornadoes (corresponding to an Enhanced Fujita scale rating of 2 or above) are indeed spawned by supercell thunderstorms (Johns and Doswell 1992). As such, it is of utmost importance to understand and study the characteristics of these storms.

Numerous studies have identified parameters which aid in forecasting the development of supercells. Parameters such as convective available potential energy (CAPE) and bulk deep-layer $(0-6 \mathrm{~km})$ vertical wind shear serve as general predictors of the broader scale support for organized deep moist convection (e.g. Johns et al. 1993; Thompson and Edwards 2000; Thompson et al. 2003; Craven and Brooks 2004). The former is a measure of conditional static instability and is positively correlated with buoyant updraft intensity (i.e., Dutton and Johnson 1976), while the latter is a key source of ambient horizontal vorticity necessary for the development of mid-level rotation in supercells (Moller et al. 1994). However, CAPE is not always high in supercell environments (Moller et al. 1994; Sherburn and Parker 2014), and therefore cannot solely be used as a predictor of tornadogenesis. 
Two parameters, however, have shown skill in distinguishing between nontornadic and tornadic supercell environments, the first of which is low-level $(0-1 \mathrm{~km})$ vertical wind shear. Climatologies utilizing proximity soundings of supercell thunderstorms have identified increased low-level shear values in tornado-producing supercells, as compared to nontornadic supercells (Rasmussen and Blanchard 1998; Thompson et al. 2003; Craven and Brooks 2004). The impact of low-level shear on the storm environment extends beyond merely shear magnitude, however, as both the depth and orientation of low-level shear can have effects on the likelihood of tornadogenesis (Guarriello et al. 2018). The second parameter most indicative of supercell tornadoes is lifting condensation level (LCL). The same climatologies that recognized increased low-level shear in tornadic supercell environments have also noted a tendency towards higher boundary layer relative humidity (RH) and consequently lower LCLs in tornadic supercell environments.

Though low-level shear and LCL have been identified as effective discriminators between nontornadic and tornadic supercell environments, the exact dynamic and thermodynamic reasons for their predictive skill have yet to be shown conclusively. It has been noted that both of these features can influence both the strength of outflow and the position of its associated circulation relative to the updraft (i.e., Brooks et al. 1994; Gillmore and Wicker 1998; McCaul and Cohen 2002; Markowski and Richardson 2011). Guarriello et al. (2018; hereafter G18) was one of the first studies to systematically look at how shear affects supercell outflow position and how this position might influence near-ground rotation. That study's simulations were initialized with the same thermodynamic profile, meaning that differences in outflow strength and propagation were largely due to alterations of the low-level shear vector. The present study will build off of those findings by varying LCL for each of the low-level shear 
orientations tested in G18. This will allow for an analysis of not only how LCL influences the strength, buoyancy, and propagation of storm outflow, but also how such influences are sensitive to the low-level wind profile. In doing so, conclusions will be drawn about the physical mechanisms which relate the shear and LCLs of tornadic supercell environments to the process of tornadogenesis itself. It is hypothesized that there exists one or more combinations of lowlevel shear orientation and LCL which allows outflow to advect the near-ground, pretornadic circulation such that it is vertically collocated with the mesocyclone aloft, thus allowing for maximum convergence and strengthening of vertical vorticity to tornado strength.

The theoretical framework and research hypotheses associated with this study are elaborated upon throughout Chapter 2. Chapter 3 outlines the model setup and methodology implemented. Results and discussion are presented in Chapter 4, and Chapter 5 offers some closing remarks on the topics at hand. 


\section{BACKGROUND}

\subsection{Supercell Structure}

Since its first iteration over 50 years ago by Browning (1964), our conceptual model of the supercell thunderstorm has been refined and used to explain the observed kinematic features of these storms. Perhaps the most widely used model is the one put forth by Lemon and Doswell (1979), shown in Figure 1.

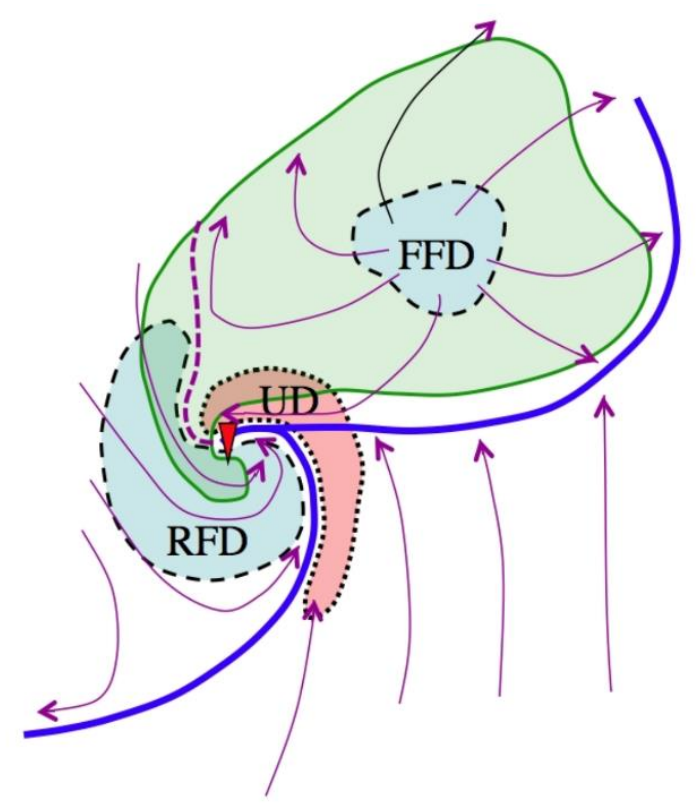

Figure 1: Plan view of a tornadic supercell, including the updraft (UD), forward flank downdraft (FFD), and rear flank downdraft (RFD) [adapted from Lemon and Doswell (1979)]

This schematic, though simple, highlights many of features that govern the growth and maintenance of supercells. Within the updraft, air is lifted to its LCL, resulting in the condensation of water vapor as well as latent heat release that is crucial to the maintenance of the updraft. The subsequent development and evaporation of precipitation leads to the formation of cool, negatively buoyant air which contributes to the formation of two distinct downdraft regions 
in a supercell (Rogers and Yau 1989). One is formed through a combination of dynamic forcing and evaporative cooling, which create a downward-accelerating area along the hook echo region referred to as the rear-flank downdraft. The other downdraft is formed as a direct result of this precipitation, which is transported downstream of the updraft by mid to upper-level winds where it melts, evaporates, and/or sublimates, leading to a broad negatively-buoyant area known as the forward-flank downdraft (Weisman and Klemp 1986; Davies-Jones 2006; Markowski and Richardson 2009). Upon reaching the surface, these downdrafts can surge out from the central storm structure in the form of cool pools (the leading edge of which is referred to as the gust front) capable of initiating new convection, generating surface circulations, and interacting with other outflow boundaries within the environment (i.e., Rotunno et al. 1988; Dowell and Bluestein 2002; James et al. 2006; Markowski and Richardson 2011). Figure 2 illustrates the complex structure and interrelationship of these features.

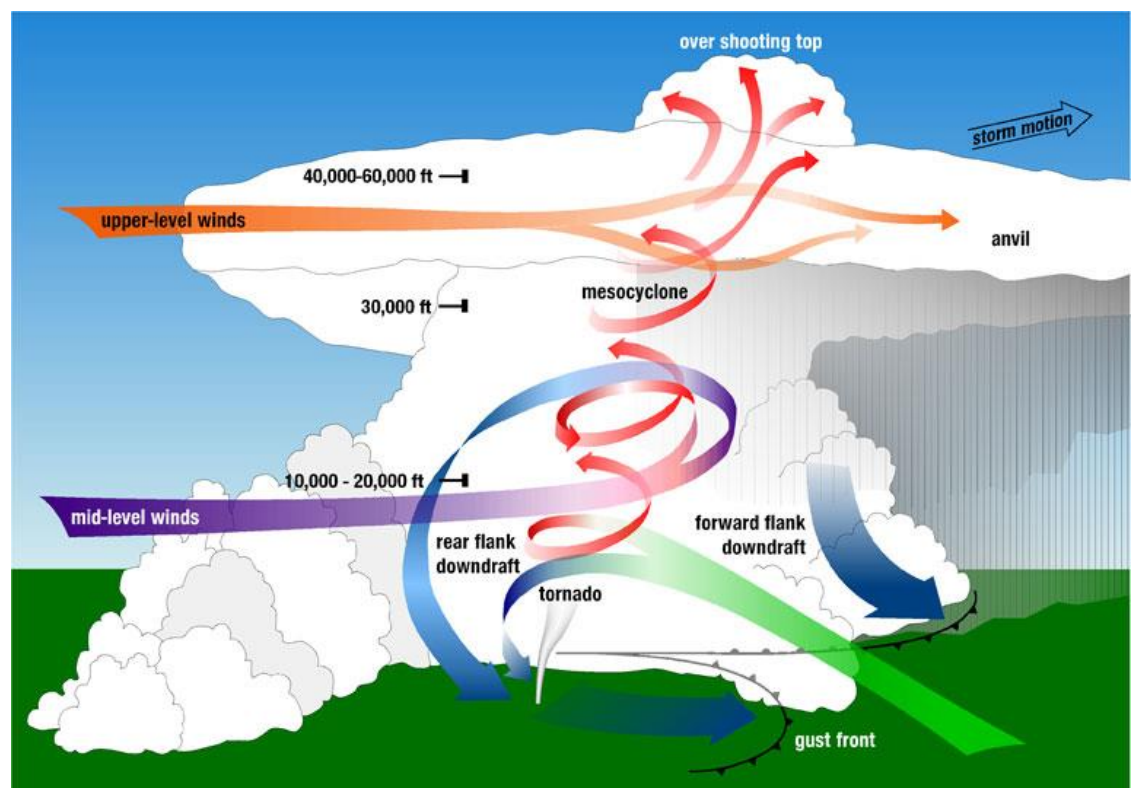

Figure 2: 3-D kinematic diagram of a tornadic supercell (NOAA National Severe Storms Laboratory, 2018) 
Though the basic structure and maintenance of supercells is largely understood, the processes which lead to tornadogenesis within a supercell are much less understood. Research within the last decade, though, has identified three essential components to forming a tornado - rotation aloft, subsequent rotation near the surface, and some mechanism to stretch surface circulation to tornado strength (Markowski and Richardson 2009). The following section will discuss the prevailing theories regarding how these components are generated within supercells and interact with one another to catalyze tornadogenesis.

\subsection{Origins of Rotation within the Supercell}

\subsubsection{Mid-level Rotation}

Given the characteristic rotation of its updraft, mid-level rotation is a necessary feature of supercell thunderstorms. This rotation is most often provided by the ample horizontal vorticity associated with ambient vertical wind shear (Markowski and Richardson 2009). When ingested into the storm updraft, this horizontal vorticity is tilted into the vertical and contributes to the mid-level rotation within the supercell updraft (i.e., Rotunno 1981; Lilly 1982; Davies-Jones 1984; Rotunno and Klemp 1985). More specifically, the updraft deforms preexisting horizontal vortex streamlines into the vertical, creating a vorticity couplet which positions itself on either side of the updraft, as shown in Figure 3 (Davies-Jones 1984). 


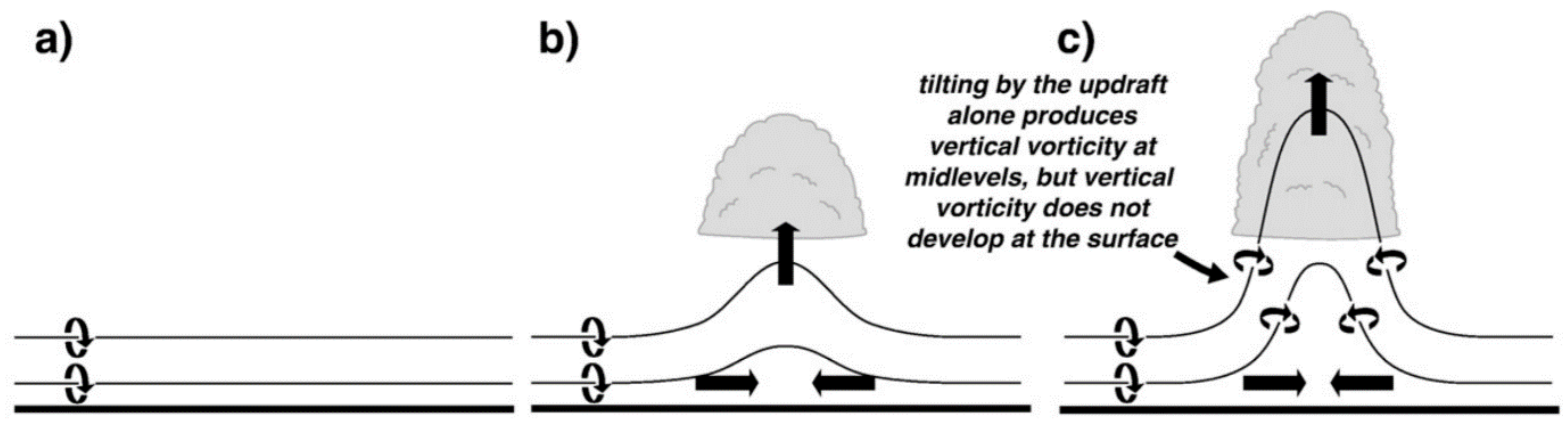

Figure 3: Simplified time evolution of vorticity streamlines in the presence of an updraft (Markowski and Richardson 2009, Fig. 2a-c)

It has been shown that the correlation between updraft strength and vertical vorticity production is stronger as the ratio of streamwise to crosswise vorticity increases, meaning that there is a larger component of the horizontal vorticity vector aligned with the storm-relative wind vector in the storm's inflow layer (Davies-Jones 1984). Looking in the direction of the ambient vertical shear, the vortex line deformation in the far right panel of Figure 3 creates an anomalous area of positive (cyclonic) vorticity to the right of the updraft and negative (anticyclonic) vorticity to the left. This vorticity configuration does not impart any net rotation to the updraft, as its associated horizontal vorticity is purely crosswise - meaning that all components of the vorticity vector are perpendicular to the environmental storm-relative wind vector (Markowski and Richardson 2009). In order for the updraft to acquire rotation, storm-relative winds must have some streamwise component that can advect the positive vorticity couplet towards the updraft core, causing the updraft to acquire cyclonic rotation (Markowski and Richardson 2011).

Numerical modeling studies have noted additional generation of rotation by way of reorientation of storm-generated, baroclinic horizontal vorticity along the forward-flank gust front (Klemp and Rotunno, 1983; Rotunno and Klemp 1985). This vorticity is largely streamwise 
because air entering the updraft from the forward flank region is often flowing perpendicular to horizontal buoyancy gradients along this forward flank, and thus can strengthen the preexisting mesocyclone aloft (Markowski and Richardson, 2009). However, when environmental vorticity generation is large and the resulting horizontal vorticity is tilted into the vertical, the role of this storm-generated vorticity within the scheme of mesocyclone development may be lessened (Shabbot and Markowski 2006).

\subsubsection{Near-Surface Rotation}

Though mid-level rotation is important for the maintenance of the mid-level mesocyclone ( 4-5 km AGL), the development of vertical vorticity near the surface is an obvious, yet crucial criterion for tornadogenesis to occur. However, unless there is appreciable near-surface circulation in the pre-storm environment - in which case this rotation can simply be converged to tornado strength - other mechanisms must be in place to either generate near-surface circulation or transport circulation-rich air towards the surface (Markowski and Richardson 2009). Though it may be tempting to attribute this to the tilting of horizontal vorticity by vertical gradients of vertical velocity, one can see from Figure 4 that this vorticity is being lifted away from the surface as it is being tilted into the vertical, making it impossible for it to contribute to circulation about a vertical axis near the surface (Markowski and Richardson 2009). 


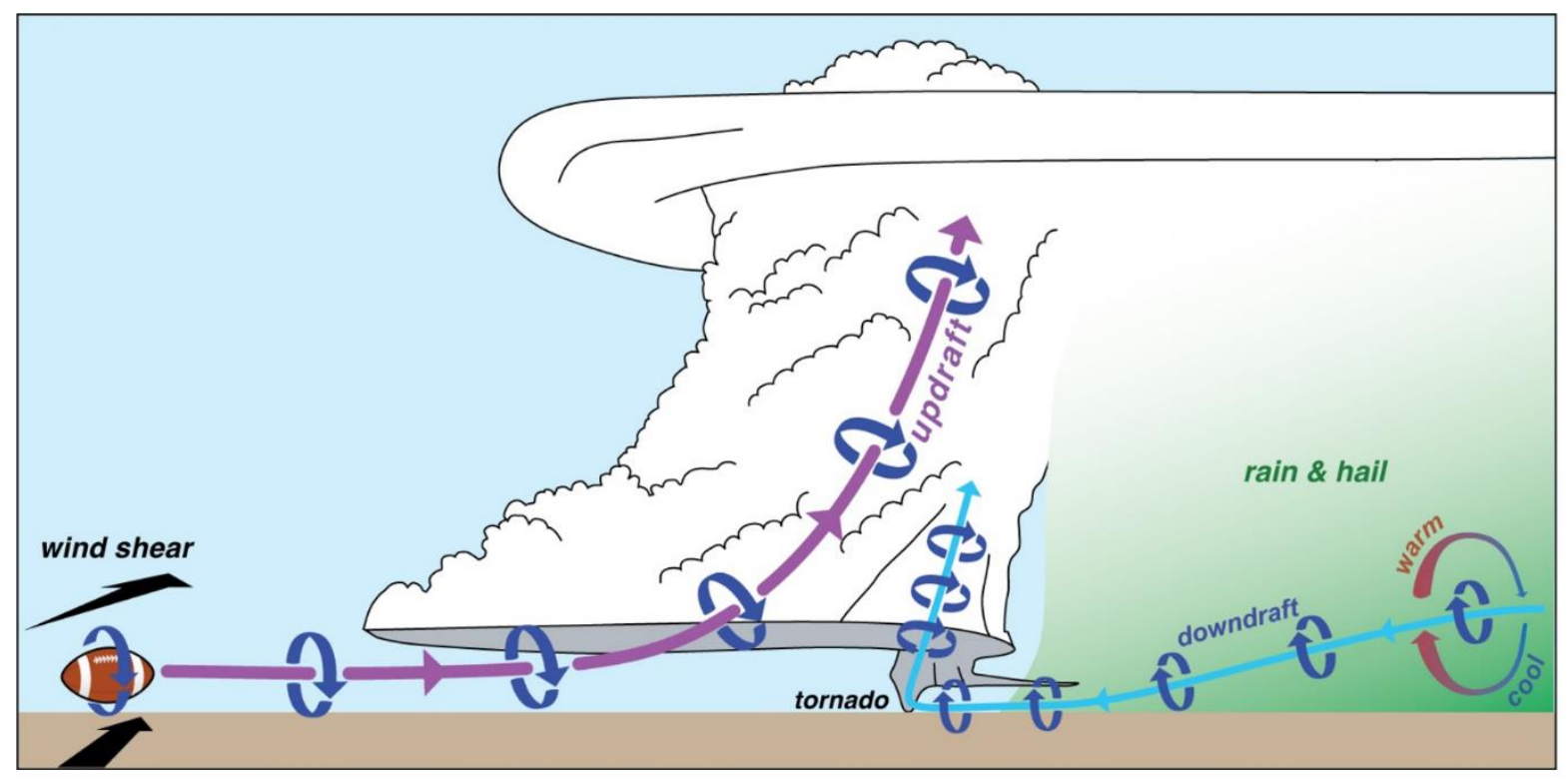

Figure 4: Diagram showing contributions of environmental shear (left side) and baroclinic overturning (right side) to vertical vorticity production in a tornadic supercell (Markowski 2018)

Therefore, vorticity aloft must be advected downwards as it is tilted into the vertical in order for near-surface vertical vorticity to exist. This can be achieved by the downdraft, if one is present during the tilting of horizontal vorticity as shown previously in Figure 3 (Davies-Jones, 1982; Davies-Jones and Brooks 1993; Davies-Jones et al. 2001). Figure 5 shows how the vortex line configuration in the far right panel of Figure 3 would evolve in the presence of a downdraft. 

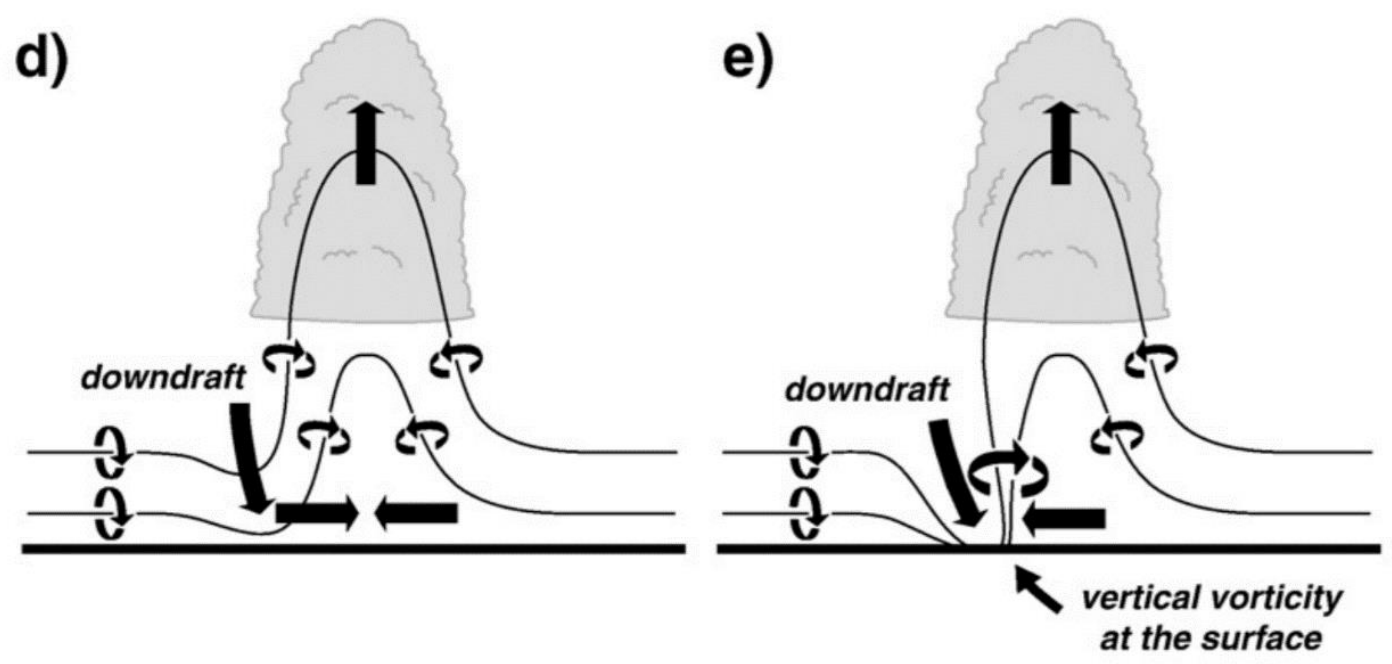

Figure 5: Simplified time evolution of vorticity streamline adjustment in the presence of a downdraft (Markowski and Richardson 2009, Fig. 2d-e)

Not only does the downdraft transport vorticity towards the surface, but it can also amplify preexisting rotation (Markowski and Richardson 2009). Despite being based on theory, the importance of the downdraft within tornadogenesis has been verified in numerous modeling experiments (Rotunno and Klemp 1985; Grasso and Cotton 1995; Alderman et al. 1999), as well as observational studies (Markowski 2002). Specifically, the prevalence of RFDs (as well as their associated hook echoes and clear slots) in the vicinity of tornadoes has been noted, with parcel trajectories often being shown to pass through the RFD before entering the tornado itself (Brandes 1978).

It can be seen from Figure 6 that as the downwind side of the vortex lines arch upward in response to the nearby low-level updraft (dynamically forced by the overlying mesocyclone), a pair of cyclonic (vortex C) and anticyclonic (vortex A) circulations form and straddle the buoyancy minimum and its associated downdraft. Thus, in a similar manner to Figure 5, baroclinic interactions within the RFD can lead to additional generation and strengthening of 
near-surface vorticity (Markowski and Richardson 2009). This dynamic lifting of baroclinic vortex lines has been noted frequently in observational studies as well (Straka et al. 2007; Markowski et al. 2008; Kosiba et al. 2013)
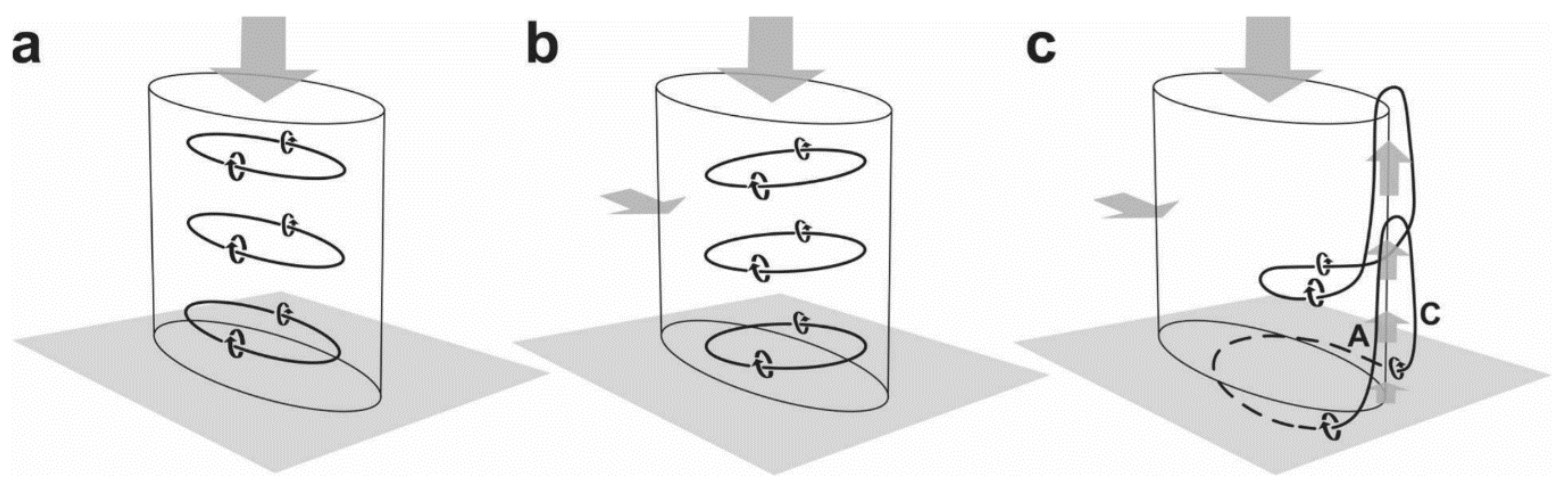

Figure 6: (a) Baroclinically generated vortex rings encircling a buoyancy minimum that extends throughout a vertical column (such a region of negative buoyancy might be found in the RFD region of a supercell, for example (b) Vortex rings being swept forward and tilted upward on their downstream sides as they descend toward the ground owing to the additional presence of rear-to-front flow through the buoyancy minimum (c) The leading edges of the vortex rings are lifted by an updraft in close proximity to the buoyancy minimum (Markowski et al. 2008, Fig. 17)

Once near-surface rotation is present within the supercell, it must be converged in order to reach tornadic strength. One proposed mechanism to do so relates to the mid-level mesocyclone, which dynamically induces a low pressure anomaly aloft as it strengthens (Markowski and Richardson 2011). This perturbation low creates an upward dynamic vertical perturbation pressure gradient force (VPPGF), which can stretch and intensify near-surface circulation (Markowski and Richardson, 2011). Since the ingestion of streamwise vorticity into 
the storm updraft is crucial for the maintenance of the mesocyclone aloft, the ambient vertical shear profile can often dictate the potential strength of the mesocyclone and its resultant VPPGF (Davies-Jones 1982; Markowski and Richardson 2014).

The ability of outflow parcels to be lifted and stretched, however, is partially depend on the buoyancy of the air within close proximity of the updraft (Markowski and Richardson 2009). Numerous studies, both numerical and observational, have noted that significantly tornadic storms often have warmer (less negatively buoyant) RFD outflow, likely due to its decreased resistance to being dynamically lifted (Markowksi et al 2002; Grzych et al. 2007; Markowski and Richardson 2009). Conversely, the existence of colder and more negatively buoyant cold pools can often have a detrimental effect on tornadogenesis. Though associated with more baroclinic generation of horizontal vorticity, this colder outflow inhibits the stretching of nearsurface air and can potentially undercut low-level circulations, thus weakening the VPPGF (Brooks et al. 1994; Gillmore and Wicker 1998, Markowski and Richardson 2014). Herein lies the "Goldilocks predicament" with storm outflow; outflow needs to be negatively buoyant enough to generate baroclinic vorticity along the forward flank gust front, but not so much so that said rotation cannot be dynamically lifted (Markowski and Richardson 2009).

Though the details of near-surface vorticity intensification are not fully understood and lie beyond the scope of this study, they highlight the profound and intertwined effect that dynamic and thermodynamic characteristics at low-levels in supercells can have on tornadogenesis. Moreover, several aspects of the near-storm environment may modulate these characteristics. The next section will specifically address how two environmental parameters low-level shear and LCL height - may interact with one another to influence whether or not a supercell becomes tornadic. 


\subsection{Influences of Low-Level Shear and LCL Height}

As with most aspects of tornadogenesis, the relationship between one variable in the storm environment and the storm's ability to produce a tornado is far from straightforward. Rather, the confluence of innumerable factors likely result in tornadogenesis. Such is the case with the two lower boundary layer features in question - low-level shear and LCL height (as it relates to outflow strength and propagation). It has long been noted that a balance between storm outflow and updraft is crucial to the maintenance of squall lines (e.g. Thorpe et al. 1972; Rotunno et al. 1988). Though this theory does not explicitly pertain to supercells, it is possible that a similar relationship holds true regarding the interaction between a supercell cold pool and the low-level shear profile, which is illustrated in Figure 7.

When a cold pool exists in an environment with no vertical shear (Figure 7b), air is swept back over the cold pool, and outflow may surge forward relative to the overlying updraft. With environmental shear and no cold pool, the storm updraft tilts down-shear (Figure 7c). Both of these setups are potentially detrimental to storm maintenance, as the slanted and weakened updraft inhibits deep convection and leads to a weaker state (Rotunno et al. 1988). However, in the optimal state where the circulations associated with the cold pool and environmental shear (Figure 7d) balance one another, an erect updraft forms down-shear of the cold pool, allowing for deep and sustained convection to persist. 
(a)

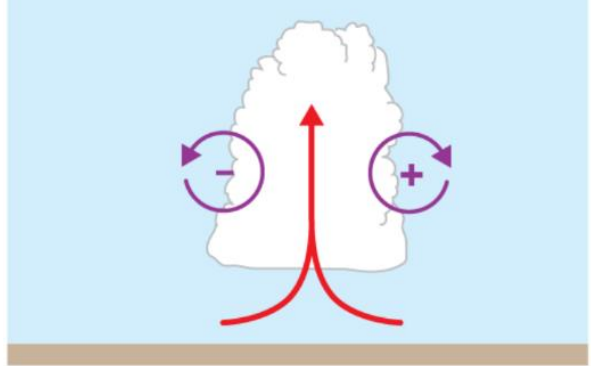

(c)

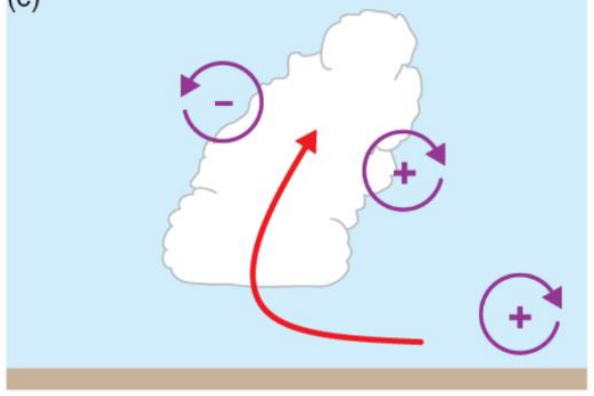

(b)

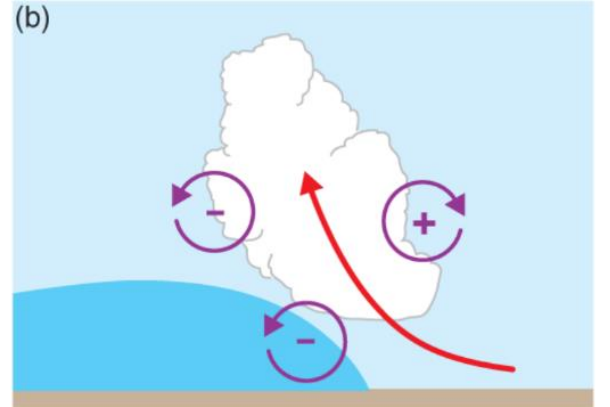

(d)

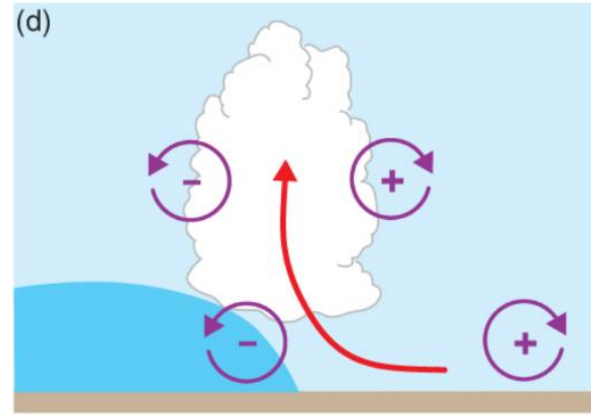

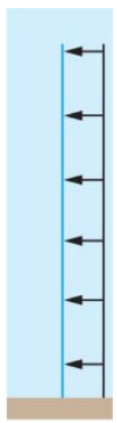

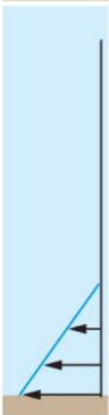

Figure 7: Diagram of the orientation of a buoyant updraft (a) without the presence of a cold pool or environmental shear, (b) in the presence of a cold pool and no environmental shear, (c) with no cold pool and environmental shear, and (d) in the presence of both a cold pool and an easterly shear profile [adapted from Figure 5 in Rotunno et al. (1988)]

In addition to the orientation of the updraft, it has also been noted that the ambient shear profile can affect the depth and propagation speed of a cold pool (i.e., Weisman and Klemp 1982; Weisman and Klemp 1984; Xue 2000; Frame and Markowski 2013). Markowski and Richardson (2011) demonstrate this point by running a series of simulations containing an eastward-moving density current in environments containing different low-level shear profiles, the results of which are displayed in Figure 8. 

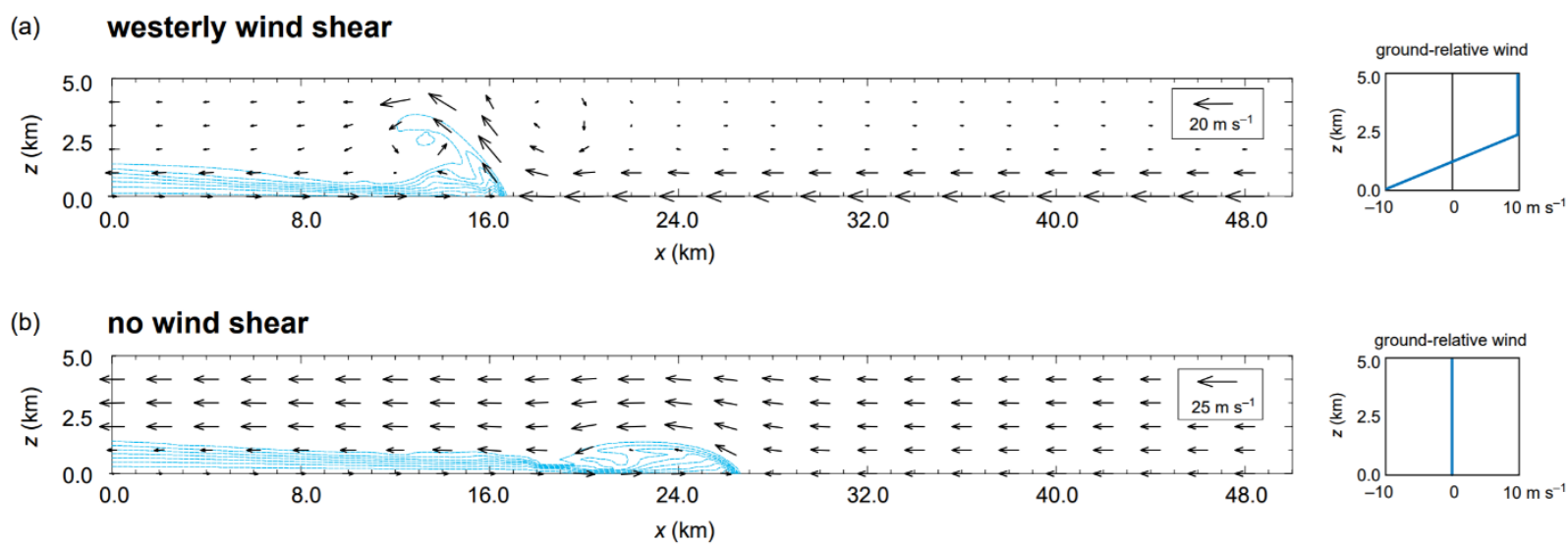

(c) easterly wind shear
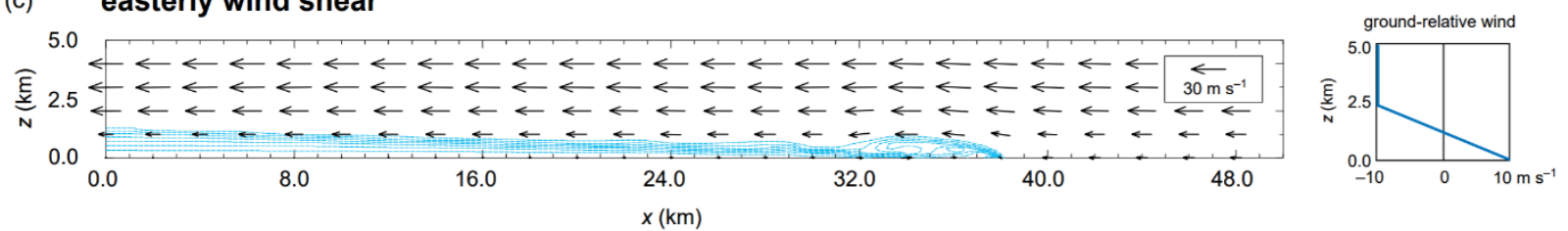

Figure 8: Numerical simulations of an eastward-moving density current in an environment containing (a) westerly wind shear, (b) no wind shear, and (c) easterly wind shear, with potential temperature perturbations contoured at $1 \mathrm{~K}$ intervals within the cold pool, starting at $-1 \mathrm{~K}$; wind vectors are relative to the density current $\left(\mathrm{U}_{\mathrm{C}} \approx 19,11\right.$, and $8 \mathrm{~m} \mathrm{~s}^{-1}$ in the easterly shear, no shear, and westerly shear cases, respectively), and the ground-relative vertical wind profiles are shown to the right of each case (Markowski and Richardson 2001, Fig. 5.31)

In Figure 8a, the low-level shear vector opposes the motion of the cold pool, resulting in a deeper and more slowly-propagating density current. In the absence of environmental shear (Figure 8b), density current-relative winds simply arch back over the cold pool without impeding its movement. Lastly, in the case of easterly wind shear when the direction of the shear vector and cold pool motion are identical, the resulting density current becomes shallower and propagates faster.

Though these studies have not focused on LCL specifically, it is important to note that outflow properties - though dependent on a number of variables ranging from the storm scale 
down to microphysical scales - are related to environmental RH (Brooks et al. 1994; Alderman and Droegemeier, 2002; van den Heever and Cotton 2004; Snook and Xue 2008), and thus LCL height. McCaul and Cohen (2002) demonstrated this relationship in an idealized environment as well, showing that lower LCLs (ie. higher boundary layer RH) produce storms that are less outflow-dominated. These findings fall in line with our conceptual understanding of LCL, where lower LCLs leads to decreased evaporative cooling as precipitation falls below cloud base, and therefore weakened outflow. Conversely, higher LCLs lead to a deeper and drier sub-cloud layer, thus increasing evaporative cooling and resulting in a more outflow-dominated storm (Markowski et al. 2002). This being said, some studies have suggested that increased CAPE and/or precipitable water values often associated with lower LCLs might alter precipitation distribution in a way that actually enhances evaporative cooling and strengthens outflow (Lerach and Cotton 2012).

Recent studies have applied a combination of these concepts to tornadogenesis, suggesting that there is an favorable positioning of a storm's cold pool and updraft/mesocyclone aloft that maximized the convergence and stretching of near-surface rotation, resulting in a rapid intensification of surface vertical vorticity (Dowell and Bluestein 2002; Marquis et al. 2012). This theory was verified to some extent by G18, who noted that surface vertical vorticity increased at a faster rate and reached greater values when the favorable dynamic low-level updraft forcing associated with the mid-level mesocyclone was located above the near-surface circulation maximum. Specifically, it was found that the depth, magnitude, and orientation of low-level shear affects the relative positioning of near-surface circulation and the mesocyclone aloft, and thus the generation of near-surface vertical vorticity. Even with the added complexity 
of varying LCL, one can still imagine an optimal state in which this vertical superposition of circulations is achieved.

\subsection{Motivation and Research Questions}

Even the brief overview of the existing literature presented above reveals the nuanced, yet critical influence that boundary layer features of the near-storm environment have on tornadogenesis. As such, our lack of understanding on why certain shear and LCL configurations favor tornadogenesis warrants a thorough investigation into its complexities. G18 has established that certain low-level shear properties allow outflow to propagate in a way that aligns surface circulations and the mesocyclone aloft such that dynamic lifting is maximized where the circulation exists, resulting in a strengthening of near-surface rotation. However, G18 did not examine variability in the ambient moisture profile, which has been shown to exert substantial influence on outflow properties, and therefore also potentially influence the positioning of circulation-rich outflow relative to the mesocyclone aloft. By altering the LCL in a set of simulations using the same low-level shear regimes as G18, this study will explore how boundary layer relative humidity influences supercell cold pool properties, especially the positioning of circulation rich outflow relative to the mesocyclone aloft, and how those properties affect the development of near-surface vertical vorticity. Moreover, this study will contextualize findings of G18, by examining how thermodynamic and kinematic properties of the near-storm environment interact to affect outflow positioning and subsequent near-surface vertical vorticity intensification. This will ultimately provide insight into the physical mechanisms causing the observed relationship that both LCL and low-level shear have with tornadogenesis. 
Through a suite of idealized numerical simulations, this study will test three overarching hypotheses regarding the role of LCL in the intensification of near-surface vorticity in supercell thunderstorms:

1. Changes in LCL will affect cold pool buoyancy in supercell thunderstorms, with higher (lower) LCLs leading to more (less) negatively buoyant outflow.

2. A lower LCL will lead to less forward propagation of outflow and embedded near-surface circulation relative to the mesocyclone aloft in supercells, compared to higher LCLs.

3. Near-surface vertical vorticity will be largest when the horizontal distance between the nearsurface circulation and the mesocyclone aloft is minimized and the dynamic vertical perturbation pressure gradient force coincident with near-surface circulation is maximized. 


\section{DATA AND METHODS}

Given the intricacies of the problem at hand, the use of idealized numerical simulations is advantageous when attempting to probe the effects of individual parameters on storm features. Though observational datasets may provide sufficient spatial detail, their temporal sparsity and limited sample size make it difficult to characterize the entire storm structure and draw overarching conclusions about the link between lower boundary layer features and tornadogenesis. Within a numerical framework, however, one can examine the full storm environment at any prescribed spatial resolution throughout the entire evolution of the supercell in question. Another advantage of a numerical modeling approach is the ability to test the influence of specific factors within the model domain. In reality, the thermodynamic and kinematic factors relevant to supercell formation and maintenance superimpose themselves on one another within the near-storm environment such that ascertaining their direct impact on the storm is difficult. However, modeling experiments can be constructed such that a specific variable is systematically altered with all else held constant, making it more straightforward to isolate its contribution to near-surface rotation within the supercell. In the case of this study, LCL height will be changed under varying low-level shear regimes, allowing for analysis of its influence on the positioning, strength, and speed of storm outflow and the subsequent dynamic implications for tornadogenesis.

\subsection{Model Setup}

The model utilized in this study is version 18 of the CM1 (Cloud Model 1; Bryan and Fritsch 2002; Bryan 2014). CM1 is a three-dimensional, non-hydrostatic, fully-compressible, 
time-dependent numerical model used to perform idealized simulations of a wide range of atmospheric phenomena including mountain waves, cold pools, large eddy simulations, and supercell thunderstorms (Bryan and Fritsch 2002). The same model configuration as G18 was implemented, in order for direct comparisons to be made between studies. The horizontal grid spacing is $500 \mathrm{~m}$, with a stretched vertical grid of 50-m spacing below $4 \mathrm{~km}$, increasing to $500-\mathrm{m}$ spacing above $15 \mathrm{~km}$. A broad domain of 350 grid points each way in the horizontal direction and 130 in the vertical was defined to capture the full storm and its environment. Each simulation was run for three hours, though most of the presented analysis focuses on the final two hours of this period to allow ample spin-up time. Adaptive time stepping is employed for the large model integration time step, with a smaller time step used to solve terms in the governing equations involving acoustic waves (Klemp and Wilhelmson 1978) in order to ensure computational stability (Mansell 2010). Rayleigh damping is applied above $15 \mathrm{~km}$ in order to absorb energy from vertically-propagating gravity waves. Not included in these simulations are Coriolis force, radiation, and surface fluxes of heat, moisture, and momentum. Model output data were stored every 5 minutes. Pertinent model specifications are summarized in Table 1.

Table 1: Table of model specifications for CM1 simulations

\begin{tabular}{|c|c|}
\hline Horizontal Grid Space & $500 \mathrm{~m}$ \\
\hline Vertical Grid Space & $\begin{array}{c}\text { Stretched grid } \\
50 \mathrm{~m} \text { below } 4 \mathrm{~km}\end{array}$ \\
& $350 \times 350$ \\
Horizontal Grid Points & 130 \\
\hline Vertical Grid Points & $20 \mathrm{~km}$ \\
\hline Model Top & \\
\hline
\end{tabular}




\begin{tabular}{|c|c|}
\hline Storm Motion & $\begin{array}{c}\mathrm{u}=17 \mathrm{~m} \mathrm{~s}^{-1} \\
\mathrm{v}=4 \mathrm{~m} \mathrm{~s}^{-1}\end{array}$ \\
\hline Run Time & $10800 \mathrm{~s}(3 \mathrm{hr})$ \\
\hline Frequency of Output & $300 \mathrm{~s}$ (5 minutes) \\
\hline $\begin{array}{l}\text { Boundary } \\
\text { Conditions }\end{array}$ & $\begin{array}{c}\text { Top/Bottom: Rigid and free-slip } \\
\text { Lateral: Open-radiative (Durran and } \\
\text { Klemp 1983) }\end{array}$ \\
\hline $\begin{array}{c}\text { Microphysics } \\
\text { Parametrization }\end{array}$ & $\begin{array}{l}\text { Morrison double moment (Morrison et } \\
\text { al. 2005) }\end{array}$ \\
\hline $\begin{array}{l}\text { Sub-Grid Turbulence } \\
\text { Scheme }\end{array}$ & 1.5-order closure (Deardorff 1980) \\
\hline Storm Initialization & $\begin{array}{l}\text { Warm bubble (maximum theta } \\
\text { perturbation of } 1 \mathrm{~K} \text { ) }\end{array}$ \\
\hline
\end{tabular}

\subsection{Model Initialization}

The initial atmospheric state for each model run is provided by an external sounding with the desired dynamic and thermodynamic properties for the purposes of this study. The methodology used to generate these soundings is outlined in the next section. Convection is initiated using a warm bubble with a horizontal radius of $10 \mathrm{~km}$ and a vertical radius of $1.4 \mathrm{~km}$, centered $1.4 \mathrm{~km}$ above the lower model surface at the horizontal center of the modeling domain. After initialization, the model is integrated for three hours. One hour into integration, each member in the suite of simulations being performed contain a supercell-like structure with an organized cold pool. As such, the entirety of the presented analyses will focus on the rightmoving supercell thunderstorm in the domain during the final two hours of integration. 


\subsubsection{Wind Profile}

Four different wind profiles are applied across three different thermodynamic profiles with varying LCLs, for a total of 12 simulations. A control run is performed for each LCL in which no additional shear depth is added at the bottom of the wind profile. The three remaining wind profiles are the same as those in G18 in which low-level shear depth, orientation, and magnitude are prescribed, while deep-layer wind profiles remain unchanged between simulations. This deep-layer profile consists of a clockwise turning quarter-circle, with unidirectional westerly shear above $2 \mathrm{~km}$ - a profile known to be supportive of supercell formation (Weisman and Klemp 1984). For the low-level profile, a shear depth (d) of $500 \mathrm{~m}$ and shear magnitude of $7 \mathrm{~m} \mathrm{~s}^{-1}$ is chosen. Three low-level shear orientations, a schematic of which is shown in Figure 9, were tested $-0^{\circ}$ (easterly), $90^{\circ}$ (southerly), and $180^{\circ}$ (westerly).

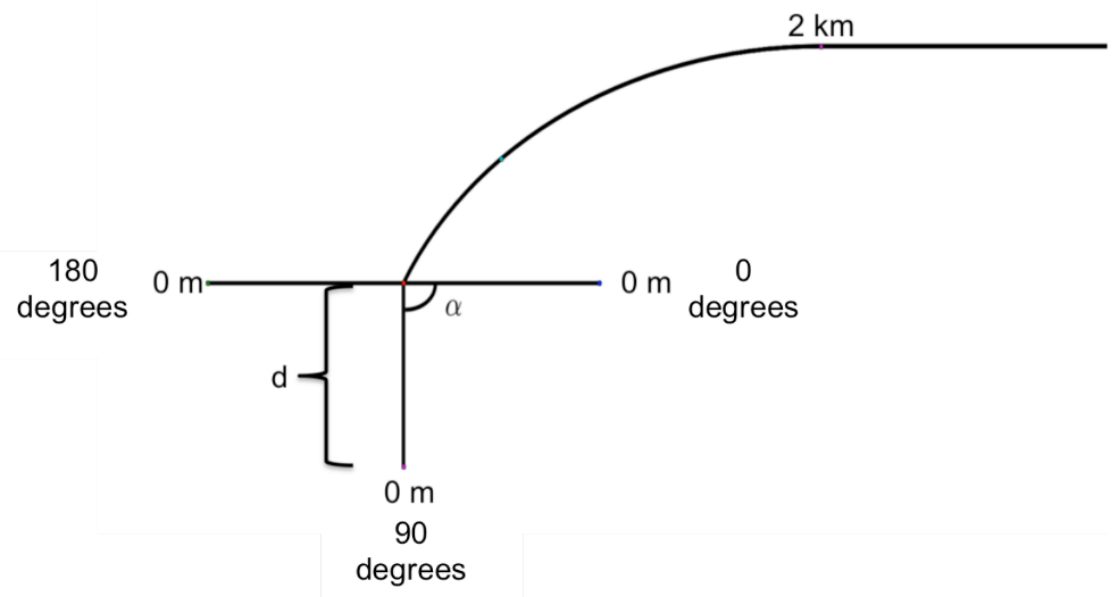

Figure 9: Schematic of model hodograph, with low-level alterations displayed [adapted from Fig. 2 in Guarriello et al. (2018)] 
The $0-3 \mathrm{~km}$ SRH values for the four input hodographs are $247,242,357,252 \mathrm{~m}^{2} \mathrm{~s}^{-2}$ for the control, $\alpha=0^{\circ}, \alpha=90^{\circ}$, and $\alpha=180^{\circ}$ hodographs, respectively. As noted in G18, the hodograph alterations were designed to minimize differences in storm-relative helicity (SRH), which is used as a proxy streamwise vorticity within the environment. Doing so allows direct inferences to be made regarding the role of low-level shear orientation on the vertical alignment of surface circulation and the mid-level mesocyclone, and the subsequent stretching and strengthening of vertical vorticity. The variability in SRH across the hodographs, particularly for the $\alpha=90^{\circ}$ case, suggest that the intensification of low-level rotation may be affected by the relative strength of low and mid-level circulations, rather than simply their vertical co-location, but this will be taken into consideration within the following analyses.

\subsubsection{Thermodynamic Profile}

Three different thermodynamic soundings are used in this study, each with a different LCL. A base sounding corresponding to the thermodynamic profile used in G18 is computed using equations adapted from Weisman and Klemp (1982), with a surface of pressure of 1000 $\mathrm{mb}$ and an approximate LCL of $1 \mathrm{~km}$. Though many methods can be used to alter the LCL height, such as changing the sub-LCL mixing ratio, this must be chosen carefully in order to limit the thermodynamic variability of the derived soundings in other aspects that may affect supercell evolution. Namely, CAPE must not vary substantially as LCL is varied, as large differences could lead to unintended differences in convection and updraft intensity. A method was developed to alter LCL while minimizing CAPE differences, in which additional layers are 
added or removed from the base sounding and the sub-LCL mixing ratio is nudged such that a surface-based parcel, when lifted to its LCL, would follow a similar moist adiabat in each sounding. A set of sample soundings is shown in Figure 10.
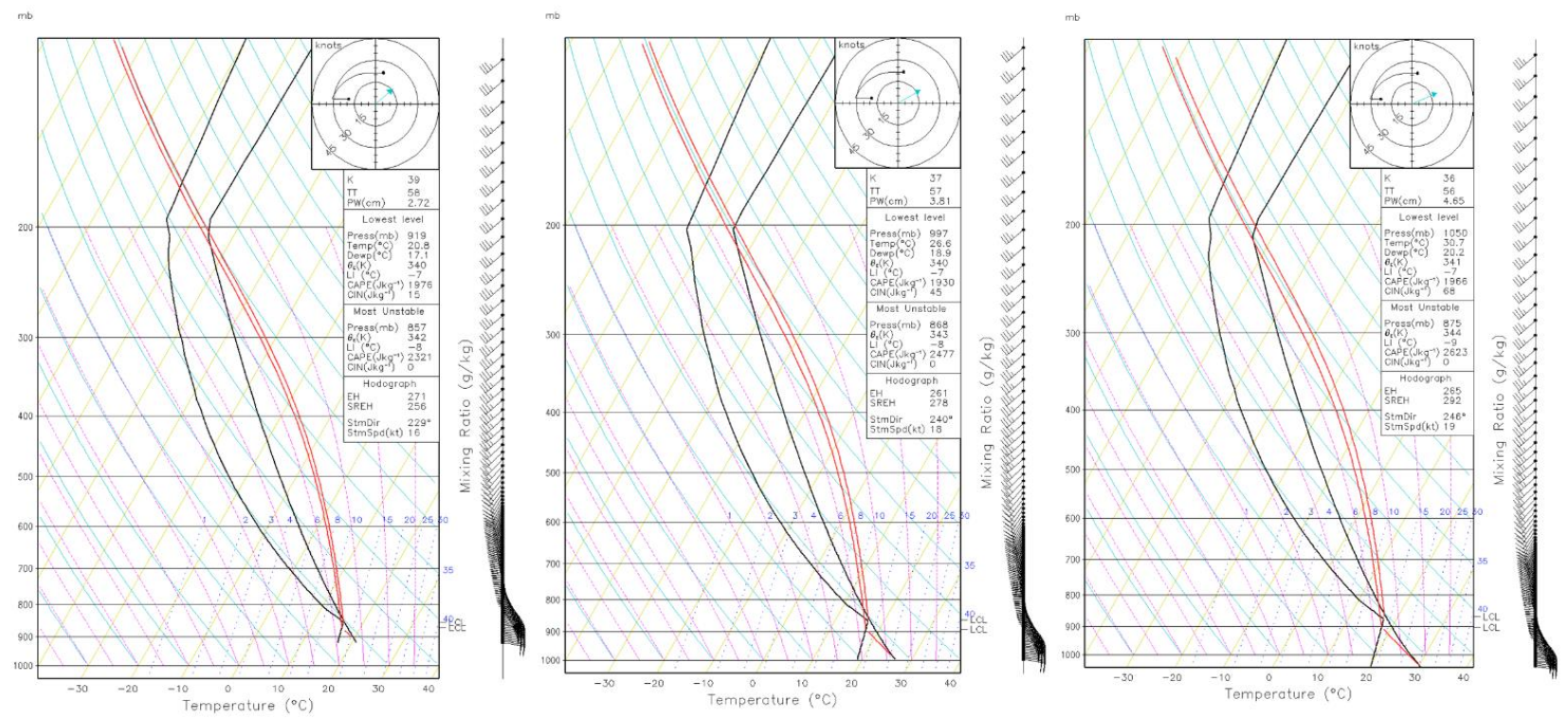

Figure 10: Sample thermodynamic soundings used for model initialization, with the low LCL configuration on the left, medium LCL in center, and high LCL on the right (with the $\alpha=0^{\circ}$ wind profile shown arbitrarily)

This process results in three thermodynamic soundings with different LCLs - approximately 0.5 , 1 , and $1.5 \mathrm{~km}$ - yet comparable CAPE values (range of CAPE values is within 10\% of their mean value). For the remainder of this study, these three soundings will be referred to as our low, medium, and high LCL cases. In addition to constraining CAPE differences, this method ensures that the thermodynamic structure above the LCL remains the same across all three soundings, minimizing variations in freezing and melting levels relative to cloud base that could have undesirable impacts on hydrometeor distribution and melting. A necessary consequence of this method is that the surface pressure of the altered soundings vary from the base sounding 
(roughly $+70 \mathrm{mb}$ for the higher LCL, and $-80 \mathrm{mb}$ for the lower LCL). The sensitivity (or lack thereof) of the simulated supercells to changes in surface pressure is discussed in the appendix. Table 2 contains additional information about the thermodynamic properties of these soundings.

Table 2: Tables of surface-based (SB), mixed layer (ML), and most unstable (MU) CAPE; SB/ML/MU convective inhibition (CIN) [all with units of $\mathrm{J} \mathrm{kg}^{-1}$; SBLCL, level of free convection (LFC), and equilibrium level (EL) [all with units of meters] in the three thermodynamic soundings

\begin{tabular}{|r|c|c|c|c|c|c|}
\hline & SBCAPE & MLCAPE & MUCAPE & SBCIN & MLCIN & MUCIN \\
\hline Low LCL & 2159 & 2188 & 2560 & -16 & -3 & 0 \\
\hline Med LCL & 2032 & 2324 & 2706 & -50 & -21 & 0 \\
\hline High LCL & 1985 & 2201 & 2847 & -78 & -50 & 0 \\
\hline AVERAGE & 2058.7 & 2237.7 & 2704.3 & & & \\
\hline RANGE & 174 & 136 & 287 & & & \\
\hline
\end{tabular}

\begin{tabular}{|c|c|c|c|}
\hline & LCL & LFC & EL \\
\hline Low LCL & 491 & 850 & 11250 \\
\hline Med LCL & 1007 & 1600 & 11933 \\
\hline High LCL & 1523 & 2251 & 12369 \\
\hline
\end{tabular}

The SBCAPE and MLCAPE differences between the three soundings are well within the prescribed $10 \%$ range. The maximum MUCAPE difference falls just outside of this range, due the fact that the method used to compute MUCAPE relies on virtual potential temperature, which is affected by the mixing ratio nudging. Also affected are surface and mixed layer CIN values, but these cannot be avoided without following a different adiabat, and thus altering CAPE and LCL height, or changing the portion of the cloud below freezing (which is undesirable).

Precipitable water (not listed) also varies between the soundings - with values of $1.13,1.55,1.94$ inches for the low, medium, and high LCLs, respectively - which may have some bearing on the outflow characteristics that will be de discussed later. 


\section{RESULTS AND DISCUSSION}

\subsection{Thermodynamic Characteristics of Outflow as a Function of LCL}

Before any conclusions can made regarding the effect of LCL on low-level rotation, it must first be determined how LCL influences the buoyancy of the outflow responsible for the positioning of said rotation within the supercell. As per the first hypothesis in Section 2.4, it is theorized that there will be an inverse relationship between LCL and cold pool buoyancy, which higher LCLs leading to more negatively buoyant outflow. The names of the model simulations according to their LCL and low-level shear orientation are listed in Table 3 for reference throughout the proceeding analyses.

Table 3: Simulation names by LCL and shear vector orientation angle $(\alpha)$

\begin{tabular}{|l|c|c|c|}
\hline & Low LCL $(\sim \mathbf{0 . 5} \mathbf{~ k m})$ & Medium LCL $(\sim \mathbf{1 ~ k m )}$ & High LCL $(\sim$ 1.5 km) \\
\hline Control & low_control & med_control & high_control \\
\hline $\boldsymbol{\alpha}=\mathbf{0}^{\circ}$ & low_ $\alpha 0$ & med_ $\alpha 0$ & high_ $\alpha 0$ \\
\hline $\boldsymbol{\alpha}=\mathbf{9 0}^{\circ}$ & low_ $\alpha 90$ & med_ $\alpha 90$ & high_ $\alpha 90$ \\
\hline $\boldsymbol{\alpha}=\mathbf{1 8 0}^{\circ}$ & low_ $\alpha 180$ & med_ $\alpha 180$ & high_ $\alpha 180$ \\
\hline
\end{tabular}

In order to judge broad spatial-temporal changes in the buoyancy field between each LCL, plan views of buoyancy were generated for the $\alpha=0^{\circ}$ and $\alpha=90^{\circ}$ simulations. Though the control and $\alpha=180^{\circ}$ simulations will be included into subsequent discussion and analysis for the sake of completeness, their storm structure will not be shown for reasons which will be discussed later. Figures 11 and 12 show hourly plots of buoyancy for the $\alpha=0^{\circ}$ and $\alpha=90^{\circ}$ simulations, respectively, within a $50 \times 30 \mathrm{~km}$ domain box (herein referred to as the storm domain) centered on the $2-5 \mathrm{~km}$ integrated updraft helicity maximum of the dominant right-moving supercell and contours of accumulated surface precipitation translated with the moving domain overlaid. The - 
$1 \mathrm{~K}$ theta perturbation displayed is used to approximate the leading edge of the cold pool, a convention that will be used in all subsequent model output figures.
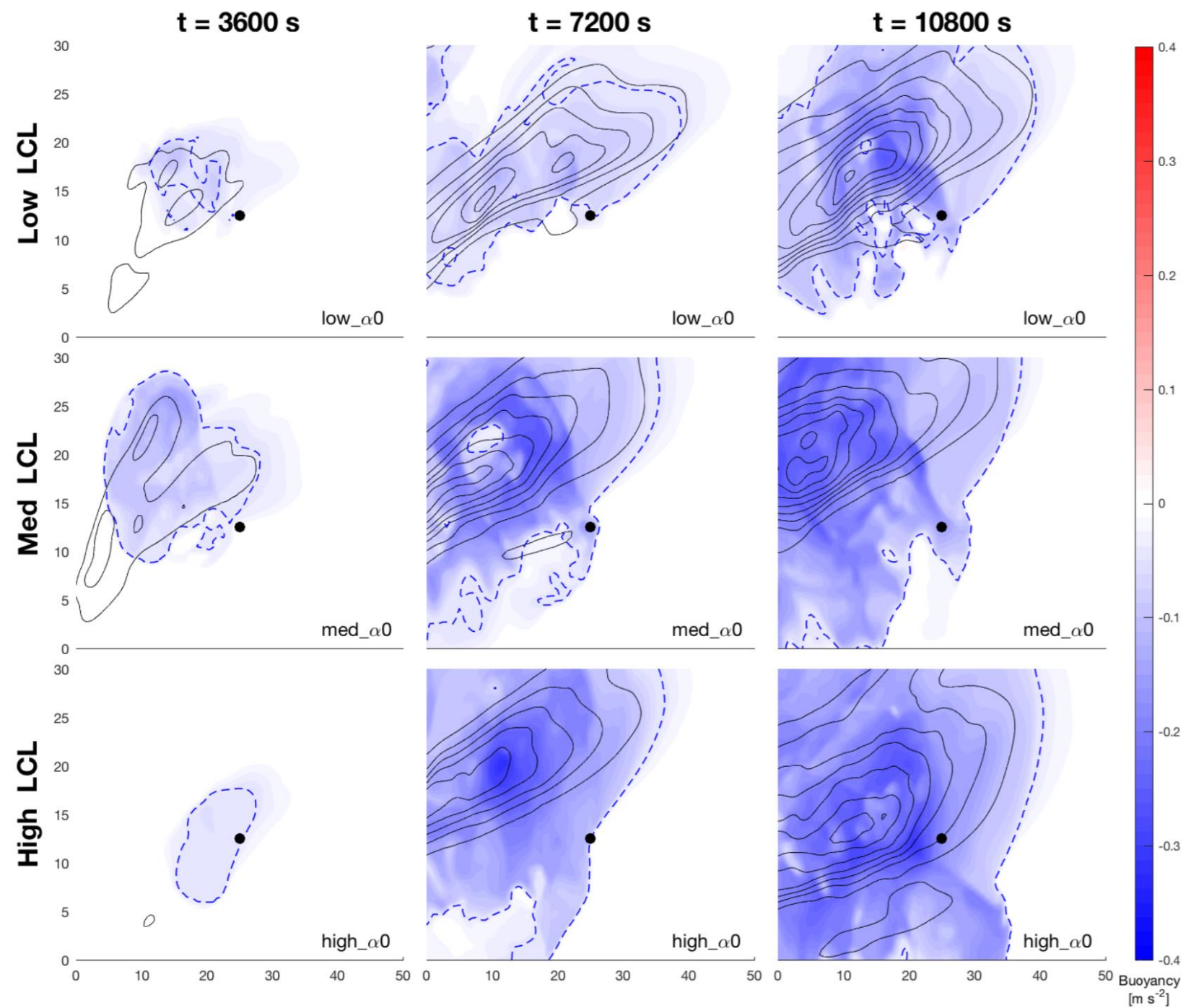

Figure 11: Hourly plots of near-surface buoyancy $\left(\mathrm{m} \mathrm{s}^{-2}\right)$ for the $\alpha=0^{\circ}$ case, with contours of accumulated precipitation translated with the moving domain in black plotted every $0.25 \mathrm{~cm}$; the black dot is the $2-5 \mathrm{~km}$ integrated updraft helicity maximum, and the blue dotted line is the contour of $-1 \mathrm{~K}$ potential temperature perturbation 


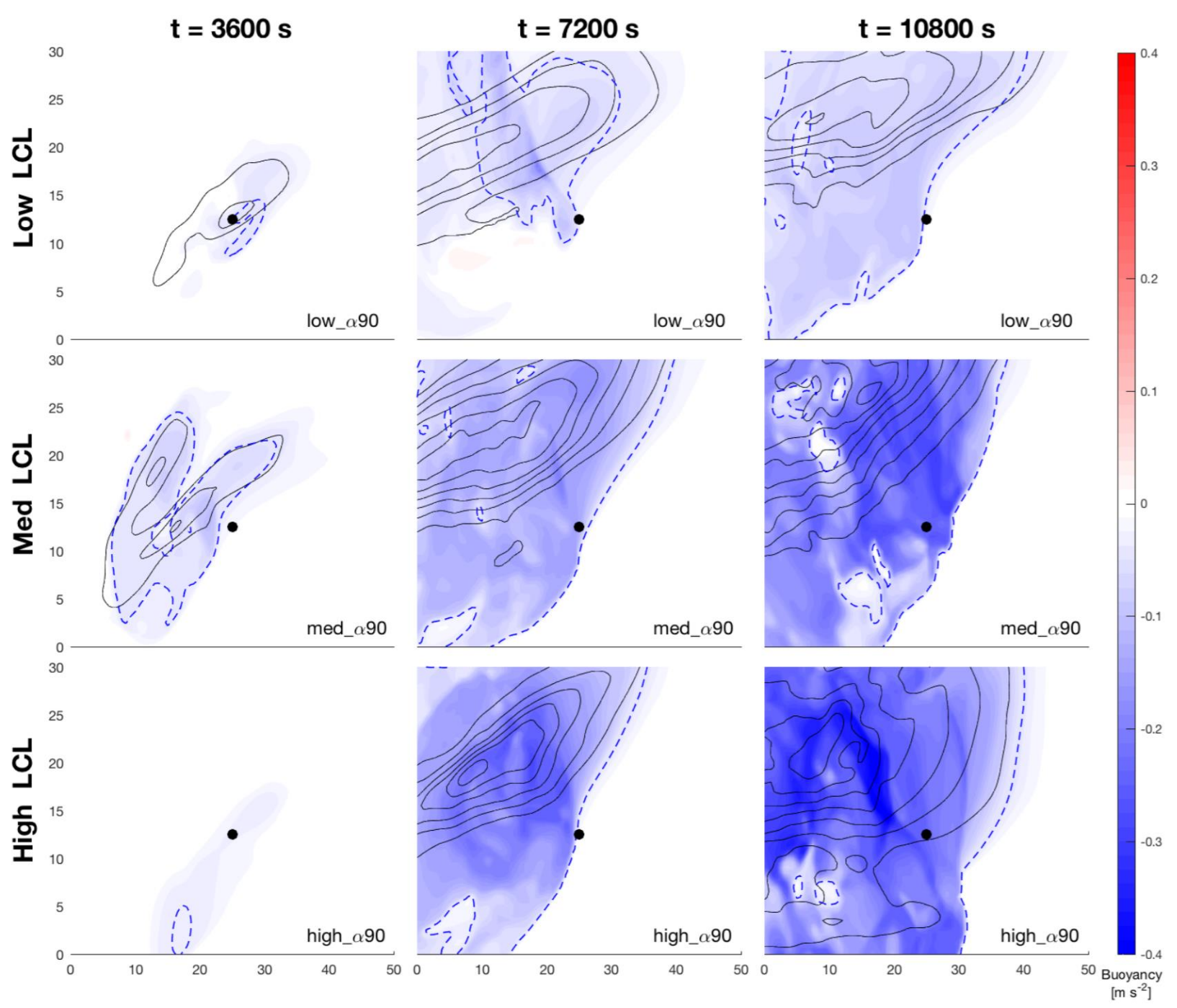

Figure 12: Same as Figure 11, but for the $\alpha=90^{\circ}$ case

Examining Figures 11 and 12, both shear orientations exhibit similar temporal patterns across LCL. In general, accumulated precipitation within the storm domain increases with time and is associated with an increasingly large and negatively buoyant cold pool, as is expected. The runs with low LCL display less negatively buoyant outflow relative to either of the higher LCLs, especially at hours 2 and 3, but the same differences are not clear in their precipitation field. The differences between medium and high LCLs are more subtle. In Figure 11, the medium and high LCLs show somewhat comparable precipitation fields, with the medium case 
showing a slightly broader area of negatively buoyant air by hour 3. By hour 2, Figure 12 shows a more negatively buoyant and broader cold pool in the high LCL runs relative to the medium LCL runs. In order to determine if the observed patterns in buoyancy represent distinct differences between the LCL simulations throughout their storm evolution, rather than instantaneous relationships at the displayed time steps, distributions of cold pool characteristics must be developed. Before these distributions are created, it must be noted that the cold pools in Figures 11 and 12 are relatively undeveloped even an hour into the simulations. This point is further communicated by Figure 13, which shows time series of average buoyancy within this inner domain for all shear orientations, as well as the $\alpha=0^{\circ}$ and $\alpha=90^{\circ}$ cases.

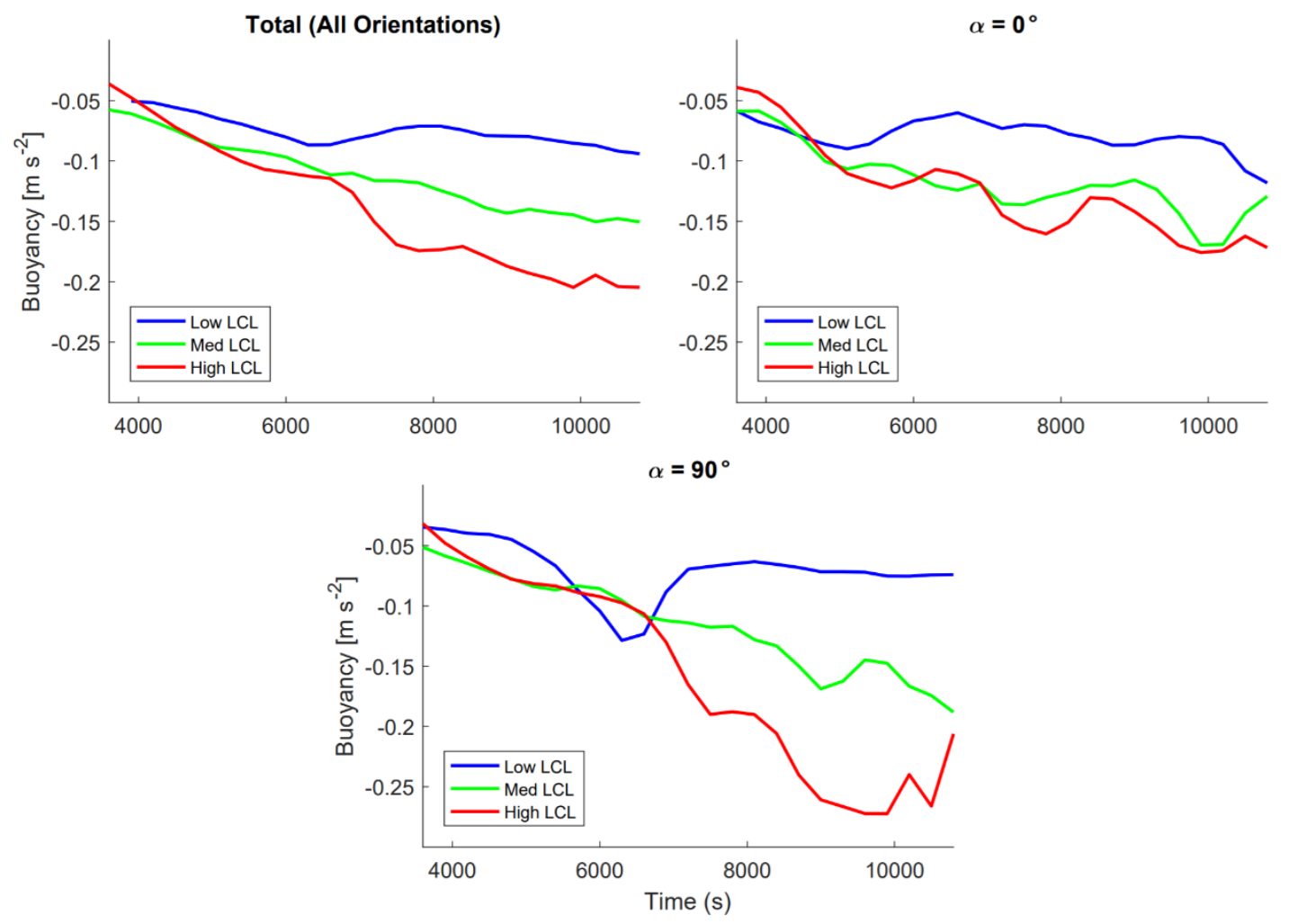

Figure 13: Time series of average cold pool buoyancy within the inner domain for each LCL across all low level shear orientations, as well as the $\alpha=0^{\circ}$ and $\alpha=90^{\circ}$ cases 
For all three plots in Figure 13, cold pool buoyancy in each LCL is relatively similar until an intermediate time step between hours 1 and 2 of model integration, at which point the buoyancy time series diverge. This divergence coincides with an increase in the fraction of the inner domain occupied by the cold pool (not shown), suggesting that this time roughly denotes when mature cold pools have developed in all of the simulations. Thus, in order to accurately probe the characteristics of this outflow and ensure that variable distributions are not low-biased simply due to varying timescales of cold pool formation, cold pool variables were only considered for the last 90 minutes of each simulation (i.e., beginning at $t=5400 \mathrm{~s}$ ). These variables - cold pool fraction (the fraction of the inner domain within the cold pool, meaning the area with potential temperature perturbations of $-1 \mathrm{~K}$ or lower), accumulated surface precipitation translated with the moving domain, and cold pool buoyancy - are examined within a smaller domain, a $22 \times 17.5 \mathrm{~km}$ box roughly centered on the updraft helicity maximum (herein referred to as the inner domain). This allows analysis to focus in on the portion of the cold pool that will specifically influence the position of near-surface circulation relevant to tornadogenesis. Box and whisker plots of these cold pool variables are shown in Figure 14. 

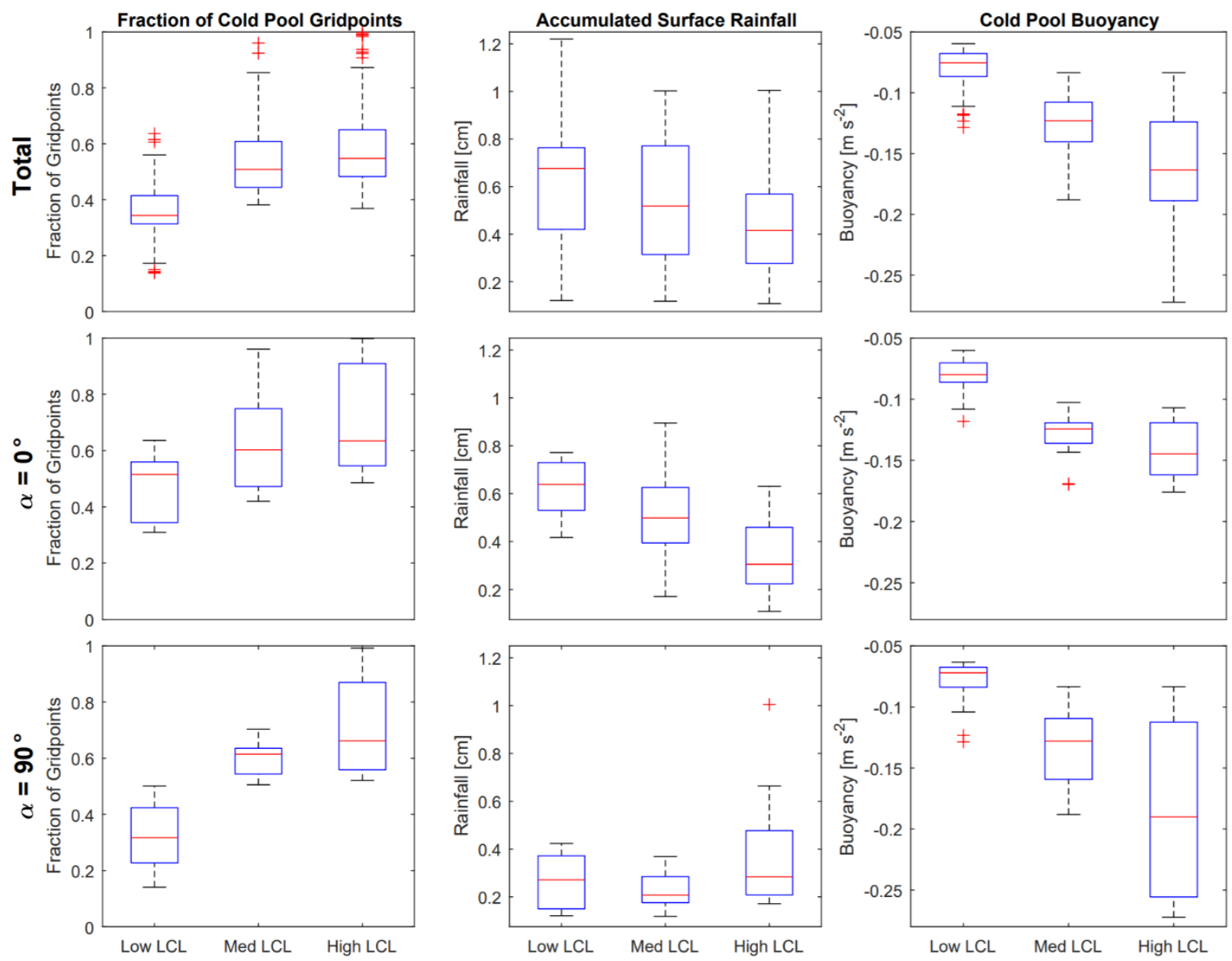

Figure 14: Box and whisker plots of the fraction of gridpoints in the cold pool (i.e., gridpoints with potential temperature perturbations of $-1 \mathrm{~K}$ or less), accumulated precipitation translated with the moving domain $(\mathrm{cm})$, and buoyancy within the cold pool $\left(\mathrm{m} \mathrm{s}^{-2}\right)$ within the inner domain during the last 90 minutes of model integration for all shear orientations, as well as the $\alpha=0^{\circ}$ and $\alpha=90^{\circ}$ cases.

Figure 14 confirms several of the observations made in Figures 11 and 12. As LCL is increased, the fraction of the inner domain occupied by the cold pool also increases, suggesting a broader and more forward-propagating cold pool. This trend is most apparent in the $\alpha=90^{\circ}$ distributions, with clear separation between the low LCL plot and the other two LCLs. In terms of accumulated surface precipitation, the differences between the LCLs are less evident, with both 
the total and $\alpha=90^{\circ}$ distributions showing substantial overlap between their precipitation distributions. The $\alpha=0^{\circ}$ distribution is the only plot to exhibit a distinct trend in accumulated precipitation, with decreasing values as LCL is increased. Initially, these findings seem counterintuitive, given that the precipitable water associated with each thermodynamic sounding actually increases with increasing LCL. One possibility is that there is simply less rain precipitating out of the storms in the high LCL simulations. Comparing the precipitation fields at the LCLs of each of the simulations (not shown), however, reveals similar reflectivity structures and magnitudes. Therefore, if higher LCLs correspond to similar or decreased surface precipitation relative to lower LCLs, there is likely increased evaporation within the sub-cloud layer. In order to gauge the plausibility of this insight, the approximate low-level relative humidity profiles for each of the thermodynamic soundings is displayed in Figure 15.

From Figure 15, the relative humidity below the LCL decreases as LCL is increased. This confirms that as LCL increases, the rain precipitating out of the storm in question is falling into a progressively drier sub-cloud layer, allowing for enhanced evaporation. If this is true, then the effects of this increased evaporation should be reflected in the buoyancy of the cold pools that develop in the simulations. Indeed, this is the case, as simulations develop increasingly negatively buoyant outflow as LCL is increased, regardless of the wind profile used. As with cold pool fraction, there is more clear separation between the low and medium LCL buoyancy distributions, but the high LCL distribution still shows a shift towards more negative values relative to the medium LCL distribution. These findings support the first hypothesis in Section 2.4 that changing LCL in supercell simulations affects the buoyancy of its associated outflow, with higher LCLs leading to the formation of a more negatively buoyant cold pool. 


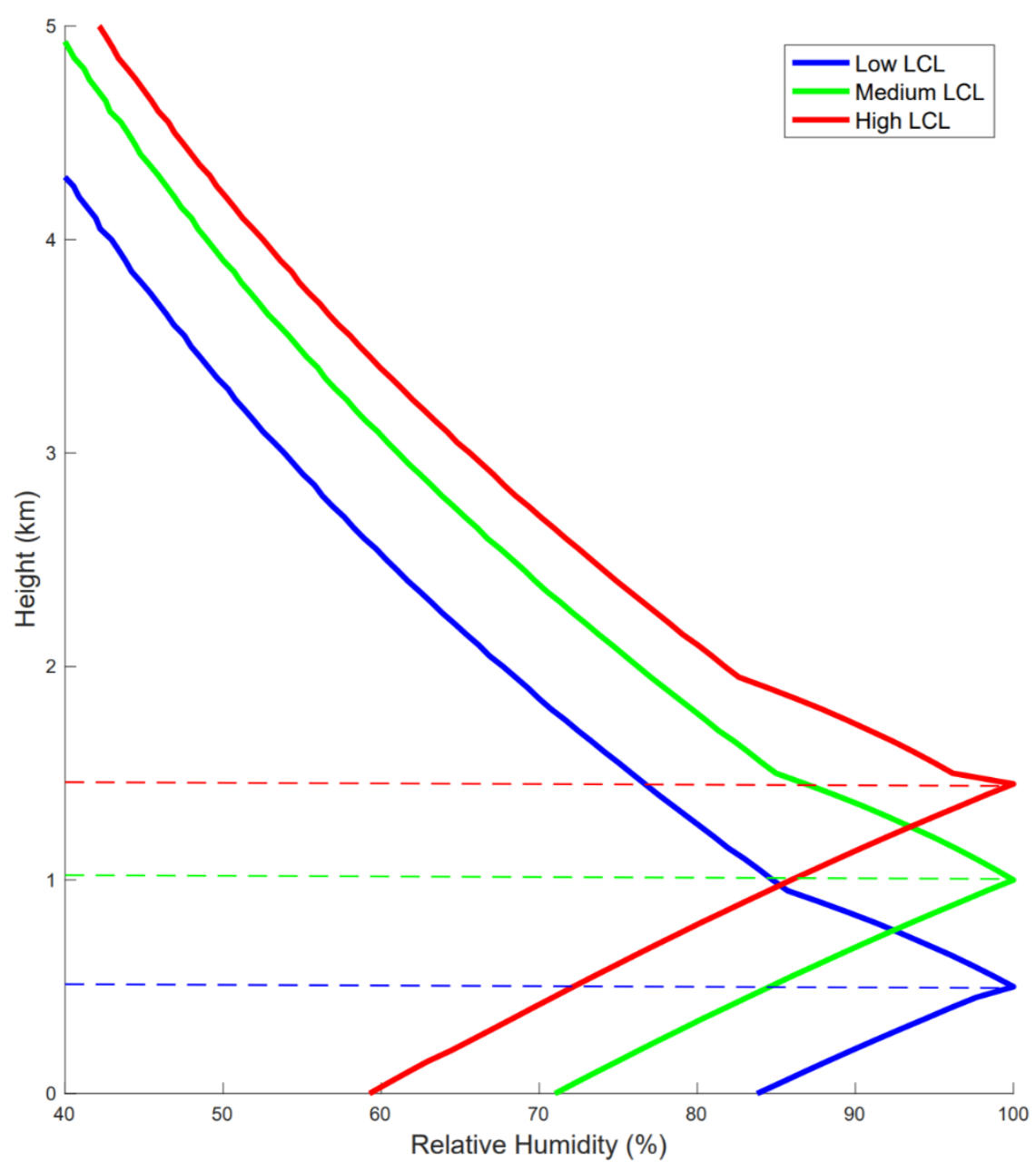

Figure 15: Relative humidity profiles within the lowest $5 \mathrm{~km}$ of each thermodynamic sounding, with dotted lines marking the approximate LCL height in each sounding

\subsection{Effect of LCL on Storm Structure and Circulation}

Now that this connection between LCL and outflow buoyancy has been established, how these variations in buoyancy influence the morphology of the simulated storms can be explored. In particular, the second hypothesis stating changes in outflow buoyancy affect the positioning of near-surface circulation relative to the mesocyclone aloft will be addressed. Near-surface 
circulation is determined by computing circulation about a horizontal, 2-km wide circuit centered on each grid point in the storm domain. By Stoke's Theorem, the circulation around a horizontal circuit is equal to the integral of vertical vorticity over the area enclosed by the circuit.

Circulation thus provides a cumulative sense of vertical vorticity in the near-surface layer, as opposed to the more local view provided by vertical vorticity alone. Markowski and Richardson (2014) note that within this context, circulation acts as a measure of broad near-surface rotation that is available to be lifted and stretched by the mesocyclone aloft. Figure 16 shows the circulation fields at hourly time steps for the $\alpha=0^{\circ}$ simulation.
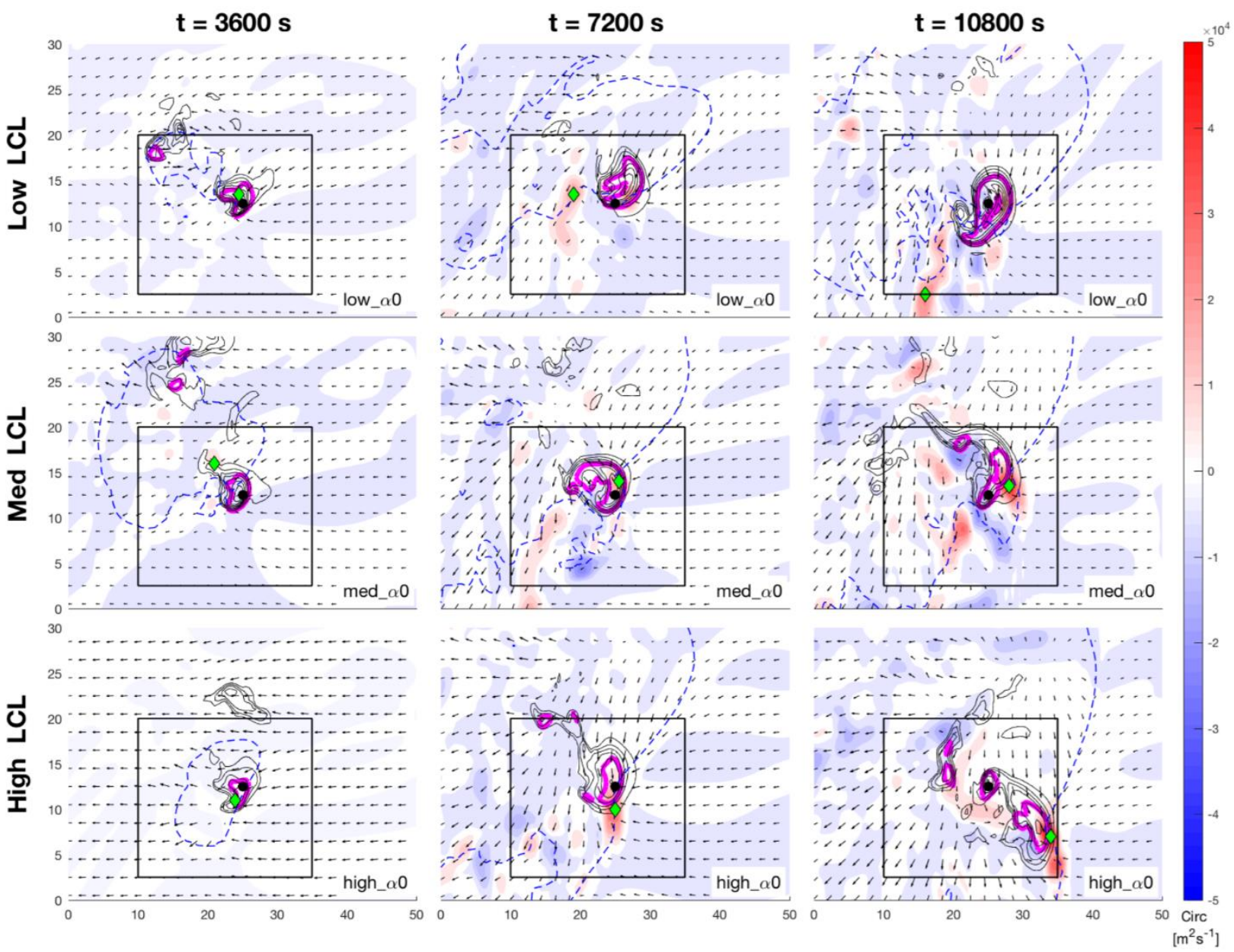

Figure 16: Hourly near-surface circulation for the $\alpha=0^{\circ}$ simulations; the dashed blue line is the $-1 \mathrm{~K}$ potential temperature contour at the lowest model grid level, black contours are mid-level (4 km AGL) 
vertical velocity plotted every $5 \mathrm{~m} \mathrm{~s}^{-1}$, the solid magenta line is the $500 \mathrm{~m}^{2} \mathrm{~s}^{-2} 2-5 \mathrm{~km}$ integrated updraft helicity contour, the black dot is the maximum updraft helicity, and the green marks the location of the maximum near-ground circulation within the inner domain introduced earlier (outlined in black); the units of the $\mathrm{x}$ and $\mathrm{y}$ axes are kilometers.

For the low_ $\alpha 0$ simulation (top row), the near-surface maximum is located beneath the mid-level mesocyclone (approximated by the $500 \mathrm{~m}^{2} \mathrm{~s}^{-2}$ updraft helicity contour) one hour into model integration. As time progresses, this circulation maximum shifts westward, as positive circulation develops behind the mid-level mesocyclone. For the med_ $\alpha 0$ simulation (middle row), the near-surface circulation maximum is constrained behind the mid-level mesocyclone at Hour 1, becomes collocated with the updraft helicity maximum beneath the mid-level mesocyclone at Hour 2, and shifts slightly ahead (eastward) of the mesocyclone by Hour 3. Lastly for the high_ $\alpha 0$ simulation (bottom row), the circulation maximum is close to the midlevel mesocyclone (at least in terms of east-west displacement) at both Hours 1 and 2. At Hour 3, this circulation is located well eastward of the updraft helicity maximum, but it remains in an area of appreciable helicity and strong mid-level updraft. These results are consistent with the second hypothesis, particularly within the context of the outflow buoyancy results, that higher LCLs lead to a more forward positioning of outflow and its embedded near-surface circulation. In order to determine whether this relationship holds under a different low-level shear orientation, Figure 17 shows the circulation fields at hourly time steps for the $\alpha=90^{\circ}$ simulation. 

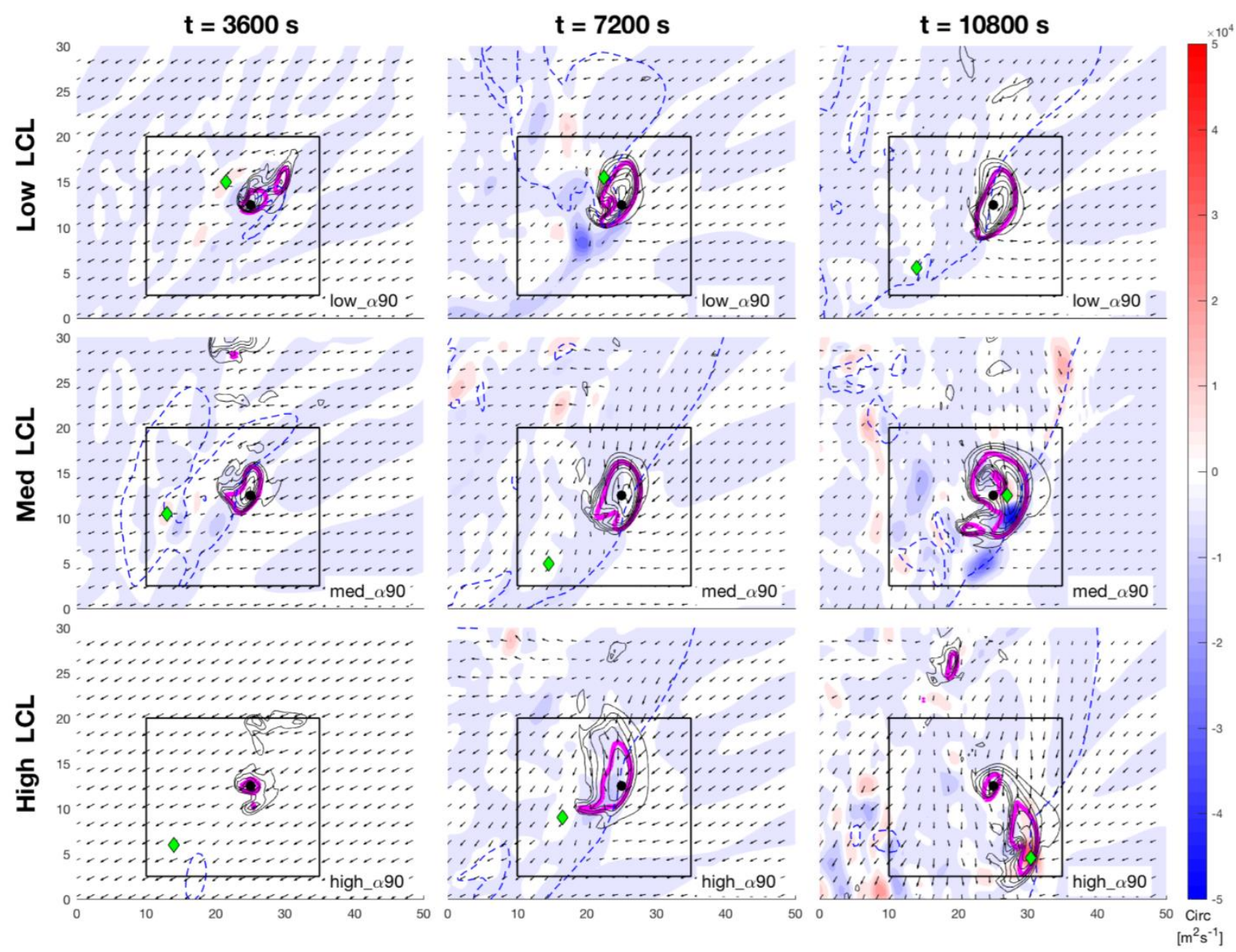

Figure 17: Same as Figure 16, but for the $\alpha=90^{\circ}$ simulations

Figure 17 displays similar patterns in the positioning of the near-surface circulation maximum as Figure 16, but the eastward progression of this maximum appears to be slower. For example, the circulation maximum in the med_ $\alpha 90$ run remains west of the mesocyclone aloft at Hour 2 and becomes collocated with the mid-level mesocyclone at Hour 3, whereas the med_ $\alpha 0$ simulation has this collocation occurring at Hour 2 and shows the circulation maximum out ahead of the mesocyclone by Hour 3. This suggests that the propagation of outflow and its embedded circulation may be sensitive to low-level shear orientation - a point that will be 
explored later. Though these figures provide a general understanding of the positioning of nearsurface circulation relative to the mid-level mesocyclone, only so much information can be gleaned from these instantaneous fields. In order to place the time steps presented within the context of the overall storm evolution, time series of maximum near-surface vertical vorticity and maximum integrated updraft helicity are shown in Figure 18, as well as a new variable meant to quantify the distance between the mid-level and surface circulation maxima - meaning the east-west displacement between the updraft helicity maximum (i.e., the black dot in Figures 16-17) and the near-surface circulation maximum (i.e., the green diamond in Figures 16-17). This quantity is herein referred to as mesocyclone separation distance (as per the methodology of G18), and is defined to be as positive (negative) when near-surface circulation is located west (east) of the mesocyclone aloft.

It bears mentioning that mesocyclone separation distance can vary rapidly (e.g., near the end of the high_ $\alpha 90$ time series) due to its sensitivity to areas of comparable near-surface vertical vorticity within close proximity of the updraft helicity maximum. Therefore, time series of this quantity cannot be interpreted as following a single coherent mesocyclone center, and must instead be interpreted instantaneously relative to other presented variables. For example, the low_ $\alpha 0$ simulation shows separation distances close to zero up until roughly $\mathrm{t}=9300 \mathrm{~s}$, at which time separation distance suddenly increases. From Figure 16, however, it can be seen that this increase is not a result of circulation embedded within the leading edge of the cold pool regressing westward behind the updraft helicity maximum, but rather a consequence of circulation developing elsewhere within the inner domain. It will be shown later, however, that these caveats do not compromise the physical significance of relationships derived using this quantity. 


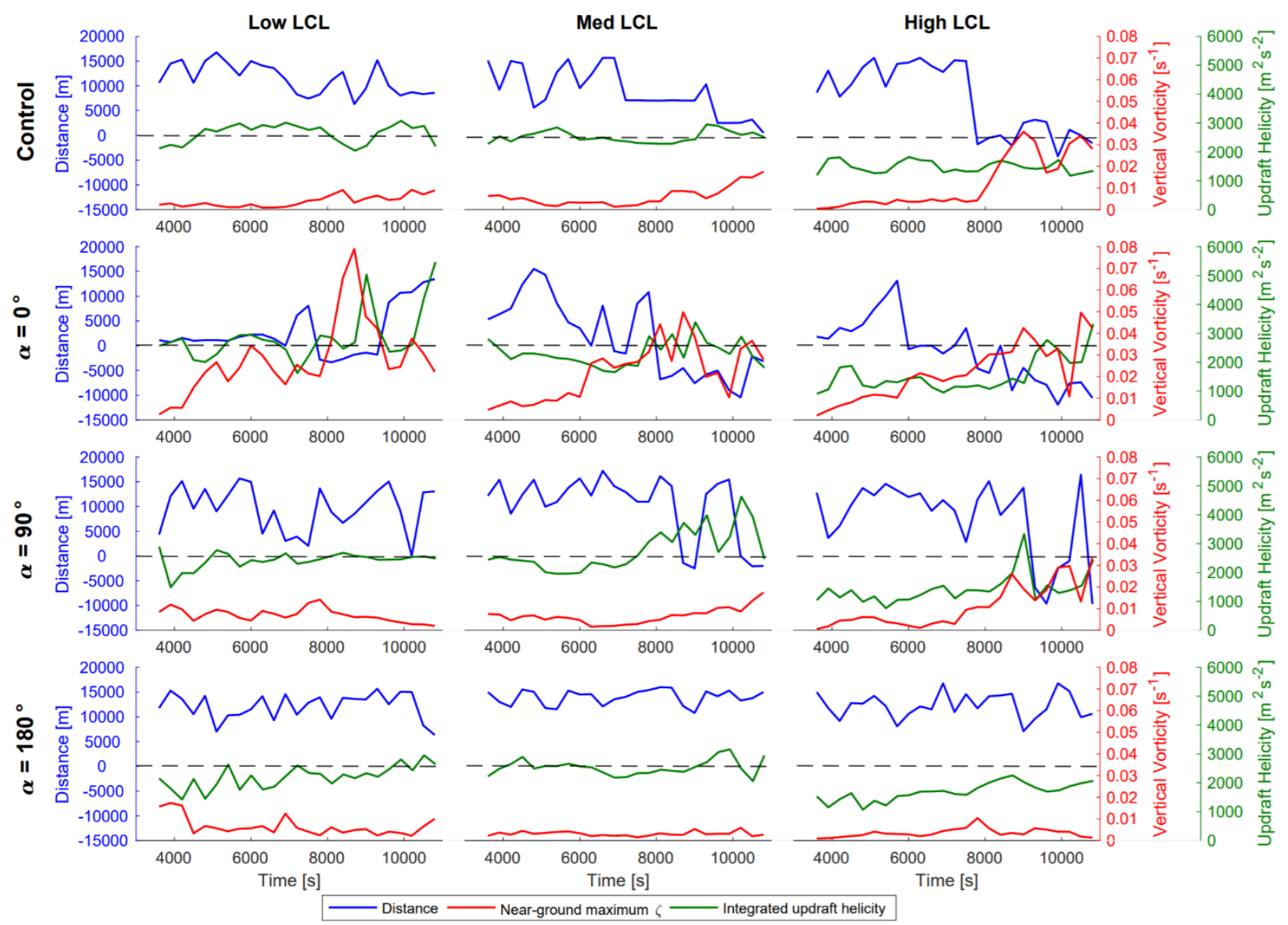

Figure 18: Time series of the mesocyclone separation distance (blue), the maximum vertical vorticity at the lowest grid level (red), and the maximum integrated updraft helicity (green) for all LCLs and low level shear orientations; the dashed black line represents the zero-line for mesocyclone separation distance

With this taken into consideration, these time series further demonstrate the observations gathered from Figures 16 and 17, that mesocyclone separation distance is sensitive to changes in LCL and, by extension, outflow buoyancy. For a given low-level shear orientation, the general trend of mesocyclone separation distances becomes more negatively sloped as LCL height 
increases. In other words, as LCL is increased and outflow becomes more negatively buoyant, near-surface circulation in transported faster downwind, relative to the mid-level mesocyclone. Additionally, all but one of the low LCL cases maintain positive mesocyclone separation distances throughout the presented hours of the model simulation, while their corresponding high LCL cases transition to large, negative distances in the latter of half of the simulation. To this end, the high LCL cases contain the most negative mesocyclone separation distances of any of the LCL configurations, for their respective low-level shear orientations. This falls directly in line with our second hypothesis that LCL influences the displacement of near-surface circulation relative to the mesocyclone aloft, with lower LCLs (and consequently less buoyant outflow) leading to a less forward positioning of near-surface circulation.

It is clear, however, that the degree of this separation is also influenced by the low-level shear profile. For example, the $\alpha=90^{\circ}$ cases display delayed and sudden decreases in separation distance, relative to the $\alpha=0^{\circ}$ cases which show earlier, more gradual transitions from positive to negative separation distance. Furthermore, the $\alpha=180^{\circ}$ cases maintain large positive mesocyclone separation distance throughout the entire simulation, regardless of their LCL. In order to quantify this low-level shear influence, it must be determined to what extent the lowlevel winds in each shear orientation oppose storm outflow. This was accomplished in a similar manner to G18, where first the average gust-front position is obtained by averaging the potential temperature perturbation at the lowest grid level over the last two hours of each simulation in a $15 \times 15 \mathrm{~km}$ subdomain centered on the $2-5 \mathrm{~km}$ integrated updraft helicity maximum at each five -minute output interval. The slope of a linear best-fit line of gridpoints where the theta perturbation is between $-1.1 \mathrm{~K}$ and $-0.9 \mathrm{~K}$ is used to compute the gust-front orientation angle, and the average $0-1 \mathrm{~km}$ storm-relative winds at these points are projected onto this average gust 
front orientation in to determine the component of these winds that oppose the gust front. The results of this process are summarized in Table 4.

Table 4: Average gust-front orientation angle (relative to a N-S orientation, with north corresponding to $0^{\circ}$ and east to $90^{\circ}$ ), and the average component of $0-1 \mathrm{~km}$ storm-relative winds opposing the gust front

\section{Gust Front Orientation Angle}

\begin{tabular}{|l|c|c|c|}
\hline & Low LCL & Medium LCL & High LCL \\
\hline Control & 56 & 52 & 39 \\
\hline $\boldsymbol{\alpha}=\mathbf{0}^{\circ}$ & 61 & 63 & 49 \\
\hline $\boldsymbol{\alpha}=\mathbf{9 0}^{\circ}$ & 52 & 43 & 43 \\
\hline $\boldsymbol{\alpha}=\mathbf{1 8 0}^{\circ}$ & 51 & 61 & 55 \\
\hline
\end{tabular}

\section{Average 0-1 km Storm-Relative Winds Opposing Gust Front (m s-1)}

\begin{tabular}{|l|c|c|c|}
\hline & Low LCL & Medium LCL & High LCL \\
\hline Control & 9.25 & 9.85 & 11.13 \\
\hline $\boldsymbol{\alpha}=\mathbf{0}^{\circ}$ & 6.31 & 6.36 & 8.68 \\
\hline $\boldsymbol{\alpha}=\mathbf{9 0}^{\circ}$ & 8.41 & 9.38 & 10.45 \\
\hline $\boldsymbol{\alpha}=\mathbf{1 8 0}^{\circ}$ & 10.38 & 9.69 & 11.23 \\
\hline
\end{tabular}

The $\alpha=0^{\circ}$ shear profile corresponds to the lowest gust front-opposing winds for each LCL, which explains the sustained decreases in separation noted in Figure 18 for this shear orientation. With weaker winds opposing the cold pool, the outflow in the $\alpha=0^{\circ}$ cases is able to propagate faster and advect near-surface circulation more rapidly than the other low-level shear orientations. This is evidenced by the sustained, negative trend in mesocyclone separation distance noted in the $\alpha=0^{\circ}$ cases (particularly the medium and high LCL configurations). As expected, the $\alpha=180^{\circ}$ profile corresponds to some of the highest gust front-opposing winds for each LCL, which act to constrain near-surface circulation well behind the mid-level mesocyclone, resulting in the large positive separation distances shown in Figure 18. The control 
and $\alpha=90^{\circ}$ profiles have similarly moderate gust front-opposing winds, which is to be expected given that their associated low-level shear vectors have more of a component parallel to their cold pools, in comparison with the $\alpha=0^{\circ}$ and $\alpha=180^{\circ}$ cases . Interestingly, just as the influences of buoyancy are regulated by low-level shear, the reverse also appears to be true. The magnitude of gust front-opposing winds increases the LCL increases (for a given low-level shear orientation). This is likely due to differences in outflow propagation resulting from these LCL variations, with faster propagating outflow encountering more opposition from the ambient wind profile, in addition to changes in gust front orientation.

The observations gleaned from this section support our second hypothesis, indicating that lower LCL leads to a less forward propagation of low-level circulation relative to the mesocyclone (i.e., more positive mesocyclone separation distances), with the added caveat that low-level shear profile can modify and regulate these buoyancy influences. The next question which follows is this: how do these differences in the alignment of mid- and low-level circulation impact the subsequent lifting and intensification of near-surface vertical vorticity?

\subsection{Effect of Circulation Overlap on Low-Level Vorticity Production}

\subsubsection{Reflectivity and Circulation Structure}

Similar to G18, the storm structure at the time of maximum near-surface vertical vorticity is considered to be the most favorable time for tornadogenesis within each simulation; although the model itself cannot resolve tornado-like vortices, it is presumed that stronger near-surface vertical vorticity is associated with a greater likelihood for tornadogenesis. Figure 19 shows the 
reflectivity structure of each circulation at the time of maximum near-surface vertical vorticity, and Figure 20 shows the circulation structure at the same times.
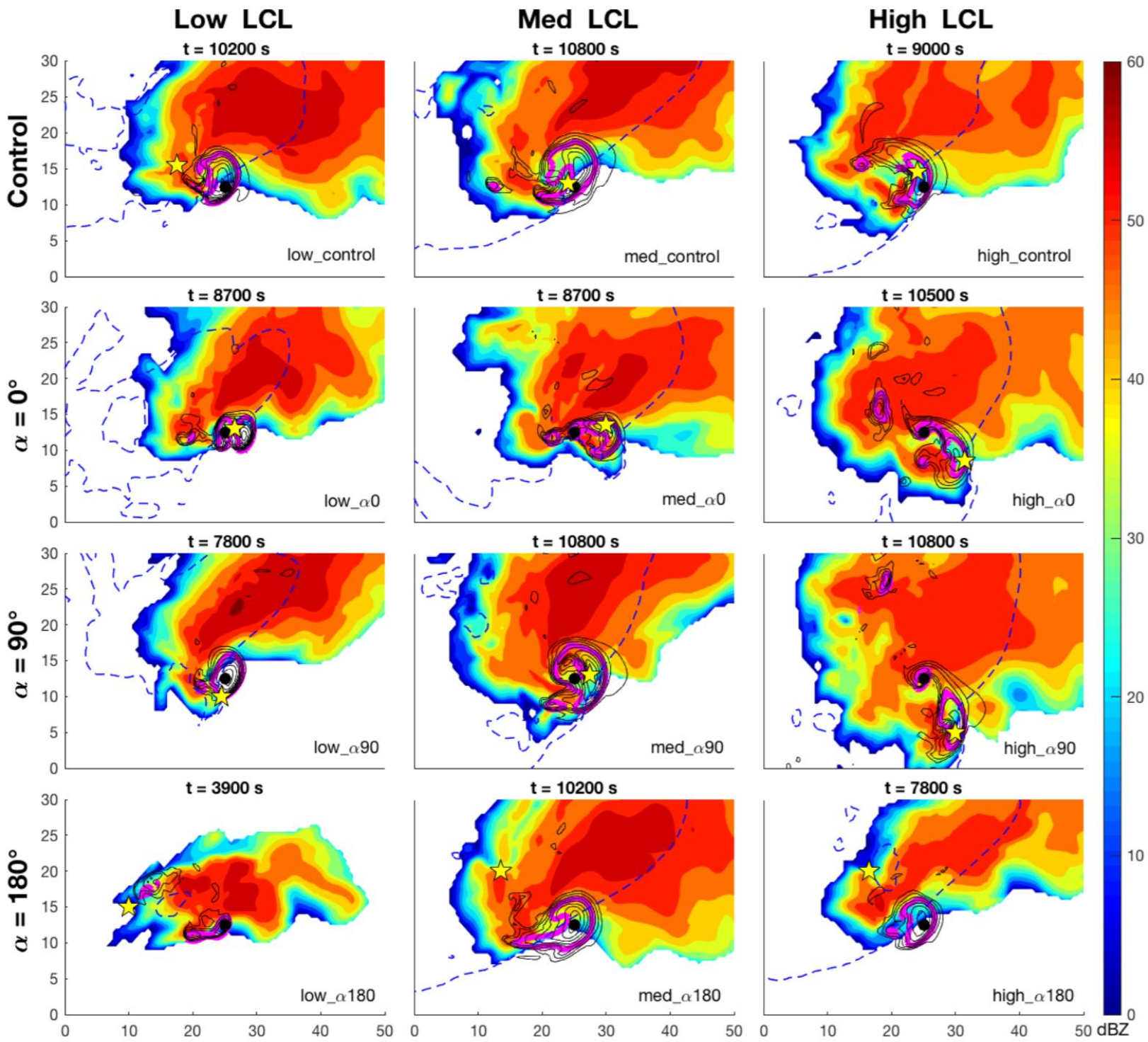

Figure 19: Reflectivity plots of storm simulations shown at the time of maximum vertical vorticity at the lowest grid level; the dashed blue line is the $-1 \mathrm{~K}$ potential temperature contour at the lowest model grid level, black contours are mid-level $(4 \mathrm{~km})$ vertical velocity plotted every $5 \mathrm{~m} \mathrm{~s}^{-1}$, the solid magenta line is the $500 \mathrm{~m}^{2} \mathrm{~s}^{-2} 2-5 \mathrm{~km}$ integrated updraft helicity contour, the black dot is the maximum updraft helicity, 
and the yellow star marks the location of the maximum near-ground (surface) vertical vorticity; the units of the $\mathrm{x}$ and $\mathrm{y}$ axes are kilometers.

Almost all of the simulations show the leading edge of their cold pool located beneath the mid-level mesocyclone at the time of maximum-near surface vertical vorticity, with the only exception being the low_ $\alpha 180$ case, which maximizes vertical vorticity before a mature cold pool has formed. Additionally, the majority of the simulations with this alignment also contain maximum near-surface vertical vorticity along the leading edge of the cold pool. These observations fall in line with those of Dowell and Bluestein (2002) and Marquis et al. (2012) that there is some favorable positioning of a storm's cold pool and updraft and/or mesocyclone aloft can maximize the convergence and stretching of near-surface rotation. In order to gauge whether this positioning is reflected in the near-surface circulation field, circulation was calculated for each of the time steps shown in Figure 19.

Several similarities between Figures 19 and 20 can be observed, with the majority of simulations containing maximum near-surface circulation beneath the mid-level mesocyclone at the presented time steps. Comparing the vertical vorticity time series in Figure 18 to the circulation configurations in Figure 20, it can be inferred that the production of near-surface vertical vorticity may be related to this positioning circulation beneath the mid-level mesocyclone (i.e., mesocyclone separation distance, as defined earlier) as well as the amount of positive circulation located beneath the mesocyclone aloft, with the simulations containing large, positive circulation beneath its mesocyclone corresponding to larger values of near-surface vertical vorticity. The next two sections will further analyze both of these possible relationships. 

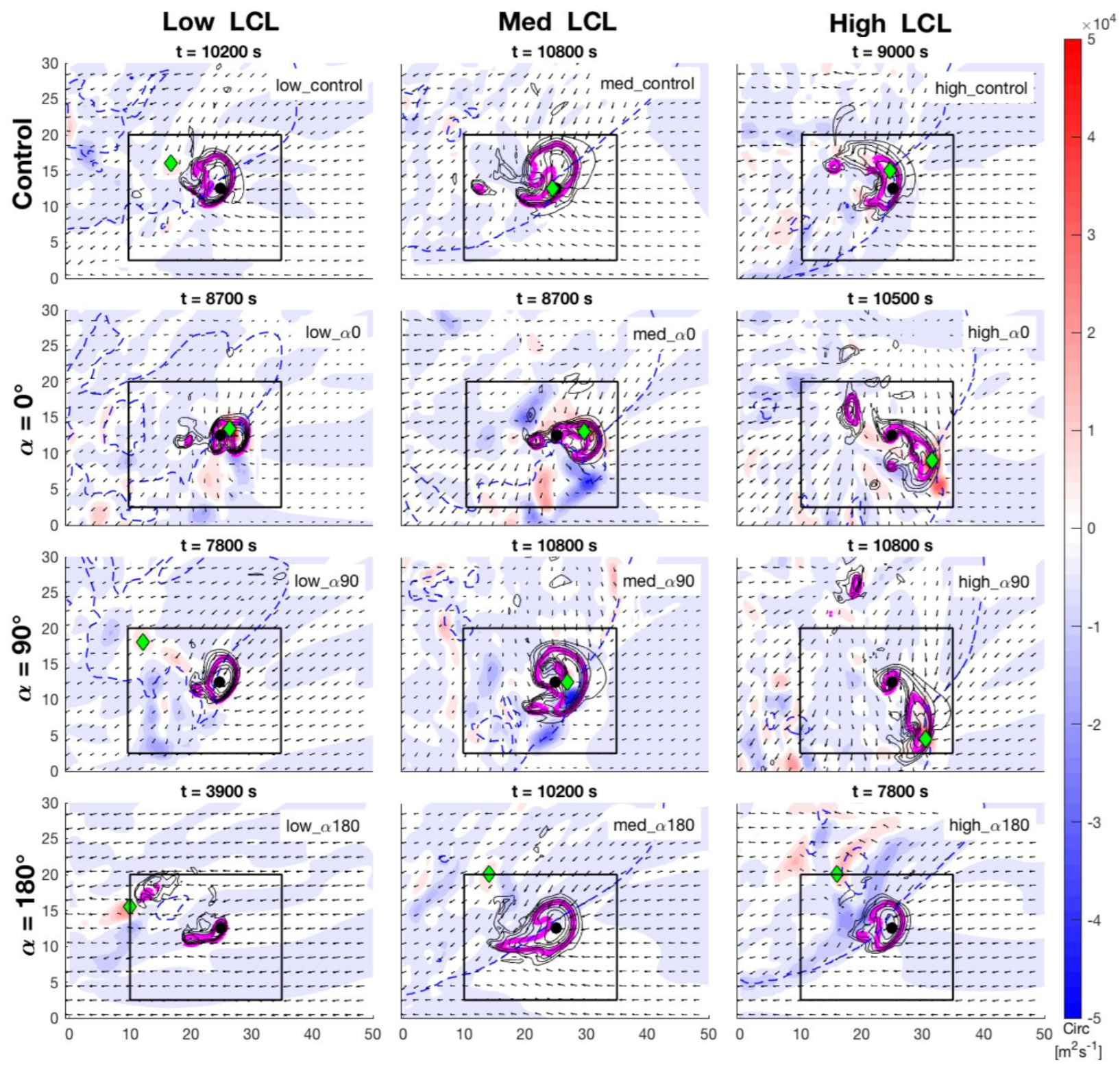

Figure 20: Surface circulation for the same simulations and time steps shown in Figure 19, with the same vertical vorticity and updraft helicity contours, and updraft helicity maxima overlaid; the wind vectors represent storm-relative flow, the black box represents the inner domain in which mesocyclone separation distance is calculated, with the green diamond representing the maximum circulation within said box. 


\subsubsection{Time Series Analysis}

Given the findings of recent studies (Dowell and Bluestein 2002; Marquis et al. 2012, G18), and in light of the third hypothesis, it is hypothesized that the collocation of low-level circulation and the mesocyclone aloft will maximize surface convergence of circulation-rich air, and thus lead to an intensification of near-surface vertical vorticity. Revisiting Figure 18, evidence of such a relationship can immediately be seen. There exists a general inverse correlation between mesocyclone separation distance and vertical vorticity across the simulations, with reductions of mesocyclone separation distance corresponding to an increase in vertical vorticity, such as in low_ $\alpha 0$ around $t=8000 \mathrm{~s}$. In the $\alpha=180^{\circ}$ simulations in which mesocyclone separation distance remains large and positive throughout the analysis period, there is minimal production of near-surface vertical vorticity, further suggesting the necessity of decreased separation distance for low-level rotation enhancement.

It is important to note that the magnitude of this increase does not appear to depend on the size of the separation reduction. For example, in low_ $\alpha 0$ there is a drop in mesocyclone distance of roughly $10000 \mathrm{~m}$ (from 5000m to $-5000 \mathrm{~m}$ ) at $7800 \mathrm{~s}$ into model integration, followed by a rapid increase in vertical vorticity of about $0.05 \mathrm{~s}^{-1}$. In high_ $\alpha 90$, there is a drop in mesocyclone at approximately 9000 s over twice the magnitude of the low_ $\alpha 0$ drop, yet only a vorticity increase of $0.015 \mathrm{~s}^{-1}$. This suggests that the dynamic mechanism responsible for this increase of near-surface vertical vorticity is more related to the reduction of mesocyclone distance, rather than the rate at which it is reduced.

The connection between updraft helicity and near-surface vertical vorticity is less clear. Updraft helicity, which can be used as proxy for mesocyclone intensity, remains relatively constant in all three of the control runs, even before and during periods of vertical vorticity 
intensification. In both the $\alpha=0^{\circ}$ and $\alpha=90^{\circ}$ runs, there does appear to be some correlation between rapids increases in helicity and vertical vorticity. That said, these increases in helicity tend to lag peaks of vertical vorticity by roughly 5-10 minutes, indicating that the relationship is driven by vorticity production at the surface (which provides circulation-air to be ingested into the updraft), rather than the dynamic forcing of the mesocyclone aloft. Additionally, this relationship could be influenced by differences in the SRH associated with each base state hodograph, and the fact that an integrated quantity such as updraft helicity will sample different sections of the vertical profile (relative to its associated LCL) due to the LCL alteration method applied.

One other observation that can be made from Figure 18 is that near-surface vertical vorticity often remains high, even after mesocyclone separation distance transitions to more negative values. Though this configuration may seem unfavorable for strengthening of nearsurface rotation, it is worth noting that the influence of mesocyclone separation may not be immediate. By the time that the impacts of decreased mesocyclone separation distance on nearsurface vertical vorticity manifest themselves within the storm structure, the near-ground rotation maximum may have already propagated out ahead of the mesocyclone aloft. Additionally, Dowell and Bluestein (2002) noted that a tornado - or in this context, intensified low-level vertical vorticity - can persist well after it is horizontally displaced from the mid-level updraft. Thus, these configurations may indeed be favorable for the intensification of low-level rotation.

The relationship between mesocyclone separation distance and near-surface vertical vorticity can be further explained by examining the maximum vertical vorticity associated with each simulation, shown in Table 5. 
Table 5: Maximum vertical vorticity at the lowest grid level for each of the model configurations (with highest vorticity for each shear orientation shown in red, and the lowest in blue)

\begin{tabular}{|l|c|c|c|}
\hline & Low LCL & Medium LCL & High LCL \\
\hline Control & $0.0091 \mathrm{~s}^{-1}$ & $0.0175 \mathrm{~s}^{-1}$ & $0.0360 \mathrm{~s}^{-1}$ \\
\hline $\boldsymbol{\alpha}=\mathbf{0}^{\circ}$ & $0.0790 \mathrm{~s}^{-1}$ & $0.0497 \mathrm{~s}^{-1}$ & $0.0496 \mathrm{~s}^{-1}$ \\
\hline $\boldsymbol{\alpha}=\mathbf{9 0}^{\circ}$ & $0.0141 \mathrm{~s}^{-1}$ & $0.0175 \mathrm{~s}^{-1}$ & $0.0334 \mathrm{~s}^{-1}$ \\
\hline $\boldsymbol{\alpha}=\mathbf{1 8 0}^{\circ}$ & $0.0174 \mathrm{~s}^{-1}$ & $0.0059 \mathrm{~s}^{-1}$ & $0.0104 \mathrm{~s}^{-1}$ \\
\hline
\end{tabular}

Comparing the observations made from Figure 18 to the values in Table 5, it can be seen that the configurations corresponding to the largest vertical vorticity values (shown in red) contain a period of near-zero mesocyclone separation distance. Conversely, the configurations corresponding to the smallest vertical vorticity values (shown in blue) contain larger mesocyclone separation distances than the other LCL configurations with their same low-level shear orientation. For all shear orientations except $\alpha=180^{\circ}$, the extrema in maximum vorticity (the red or blue highlighted values) are found in either the low or high LCL cases, suggesting that LCL has a distinct influence in the processes which dictate the formation and strengthening of this vorticity.

The importance of low-level shear orientation also is clear in this table. For $\alpha=0^{\circ}$, the low LCL case corresponds to the largest vorticity value (for the given $\alpha$ ) and the high LCL case contains the smallest vorticity value. For $\alpha=90^{\circ}$, however, this relationship is reversed, with the high LCL case corresponding to the largest vorticity maximum. Similarly, the $\alpha=0^{\circ}$ runs contain the three largest values in Table 5 - which falls in line with the surprising conclusions of G18 regarding the dynamic favorability of the $\alpha=0^{\circ}$ wind profile - while the $\alpha=180^{\circ}$ runs contain three of four lowest vorticity values. This implies that certain low-level shear orientations may modulate the effects of varying LCL on storm dynamics, possibly by 
constraining or favoring the propagation of negatively buoyant outflow responsible for advecting low-level circulation beneath the mid-level mesocyclone.

\subsubsection{Distributions of Derived Variables}

The data from each simulation are now analyzed for statistical relationships between the variables that were discussed qualitatively up to this point. As with the preceding analyses, statistics will only be performed on the model variables collected during the final two hours of each simulation. Though all shear orientations will be included in the presented statistics, particular focus will be given to the $\alpha=0^{\circ}$ and $\alpha=90^{\circ}$ shear profiles, as they are the most similar to what might be observed in nature. The control shear profile does develop intense nearsurface vertical vorticity, but its lack of low-level shear is unrealistic given typical supercell environments, which limits the physical implications of its results. As discussed earlier, the $\alpha=$ $180^{\circ}$ profile fails to develop strong vertical vorticity altogether as its intense gust front-opposing winds constrains the low-level circulation far behind the mesocyclone.

Figure 21 shows a box and whisker plot of the derived variables that have been discussed thus far in out analyses, as well as a new variable referred to as circulation fraction, for all lowlevel shear orientations. This quantity is defined as the fraction of gridpoints with near-surface circulation exceeding $5,000 \mathrm{~m}^{2} \mathrm{~s}^{-1}$ beneath the low-level mesocyclone. This metric provides a slightly different perspective than the mesocyclone separation distance by quantifying the overlap of the low-level mesocyclone and areas of appreciable near-surface circulation, which is important for the dynamic uplift and stretching of near-surface rotation. 


\section{Total (All Orientations)}
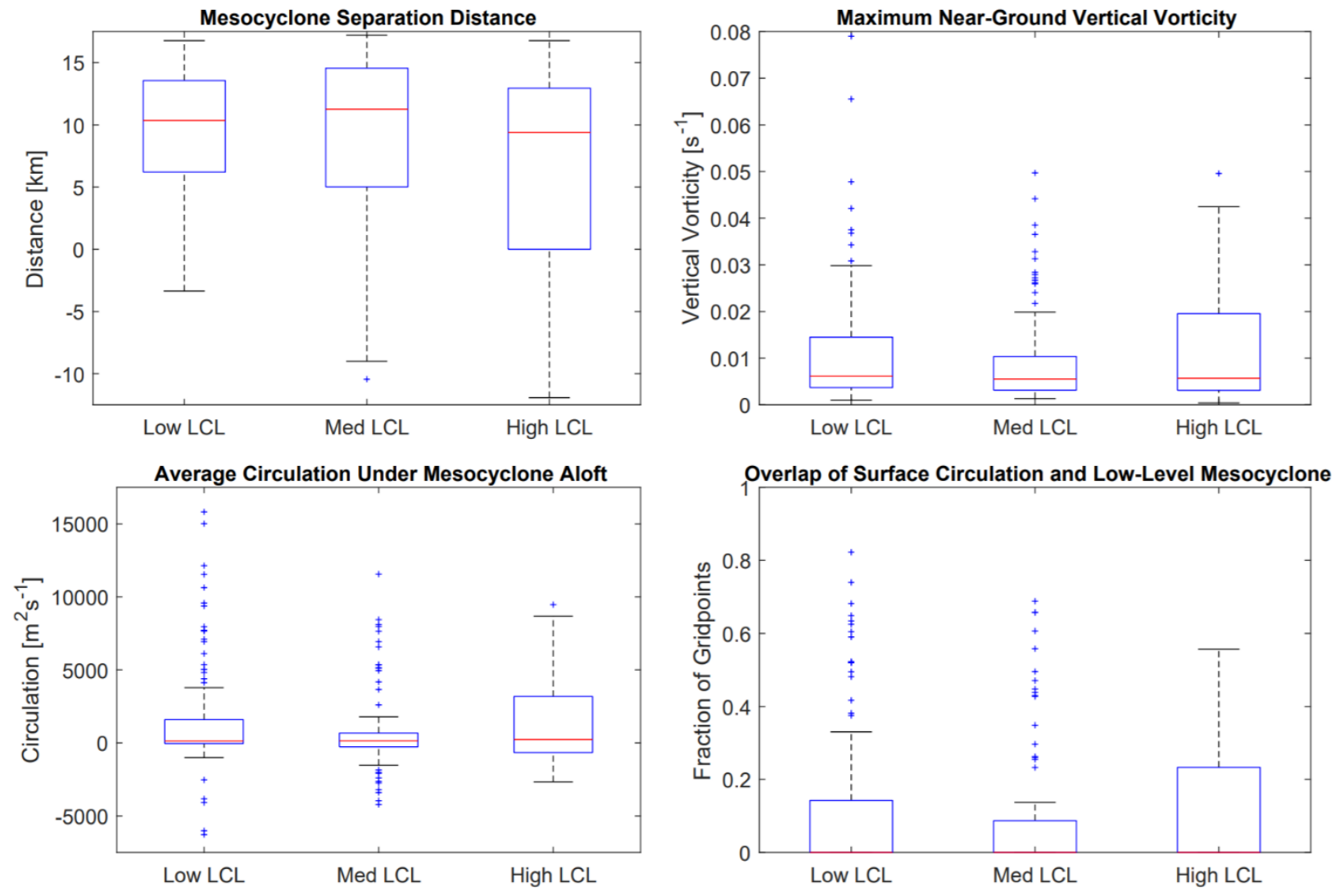

Figure 21: Box and whisker plots of mesocyclone separation distance, maximum vertical vorticity at the lowest grid level, average circulation under the mid-level mesocyclone (i.e., circulation beneath the 500 $\mathrm{m}^{2} \mathrm{~s}^{-2}$ updraft helicity contour shown in earlier figures), and circulation fraction as a function of LCL for all low-level shear orientations

Starting with the top left panel of Figure 21, there is not a clear separation in the distributions of mesocyclone separation distance across the various LCLs, with all three distributions centered on large, positive distances (possibly due to the influence of the $\alpha=$ $180^{\circ}$ runs). As LCL increases, however, the interquartile range stretches downwards towards lower values, with the tail extending into negative values. This may be an indication that outflow and the embedded low-level circulation have a tendency to surge forward of the mesocyclone 
aloft as the LCL is increased, which would be consistent with the observed trends towards more negatively buoyant cold pools with increasing LCL. Moving to maximum vertical vorticity, again there does not appear to be substantial difference amongst the three distributions, though the low LCL distribution contains two anomalously high outliers caused by the intense vorticity in low_ $\alpha 0$. The average circulation and circulation fraction distributions follow a similar pattern, with minimal variability between the LCL distributions, apart from slightly larger outliers in the low LCL cases. Some of this overlap can be attributed to the fact that for a given low-level shear orientation, different LCLs are favorable for the development of near-surface vertical vorticity. Additionally, the distributions contain data during the final two hours of model integration, so it is difficult to infer relationships between the presented quantities, such as if a reduction in mesocyclone separation distance corresponds to an increase in near-surface vertical vorticity.

In order to account for overlap related to shear orientation, the same distributions can be constructed specifically for the $\alpha=0^{\circ}$ and $\alpha=90^{\circ}$ simulations, and are displayed in Figures 22 and 23, respectively. As expected, both of these distributions show smaller lower mesocyclone separation distances, stronger circulation beneath the mesocyclone aloft, and increased overlap between the low-level mesocyclone and near-surface circulation for the LCLs corresponding to the largest near-surface vertical vorticity maxima in (cf. Table 7), supporting the hypothesis that there is a positioning of circulations near the surface and aloft that leads to stronger near-surface vertical vorticity. Furthermore, the $\alpha=0^{\circ}$ distributions - which contain the three largest nearsurface vertical vorticity values - display closer alignment between near-surface circulation and the low and mid-level mesocyclones, and stronger circulation beneath the low-level mesocyclone in comparison to the total and $\alpha=90^{\circ}$ distributions. These characteristics may be related to the weaker gust-front opposing winds associated with the $\alpha=0^{\circ}$ simulations, which could allow for 
a more forward propagation of the cold pool and its embedded near-surface circulation beneath the mesocyclone aloft.

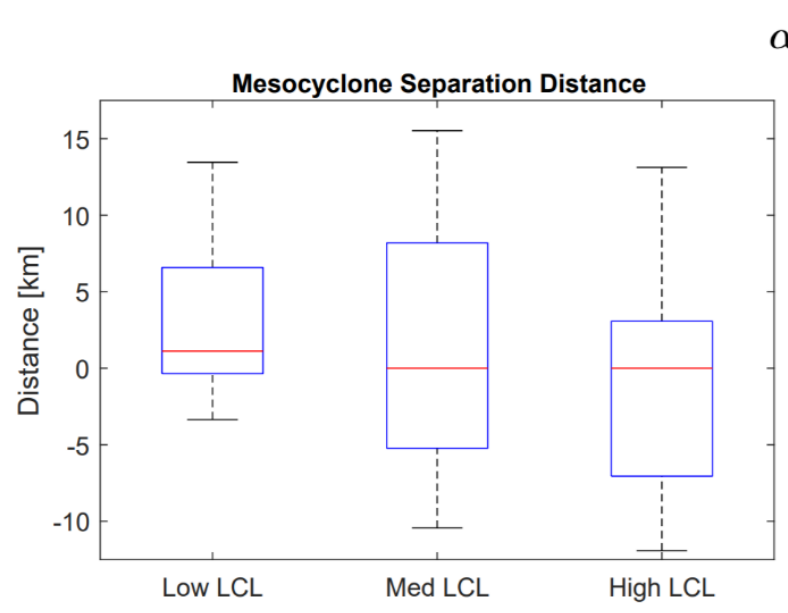

$$
\alpha=0^{\circ}
$$
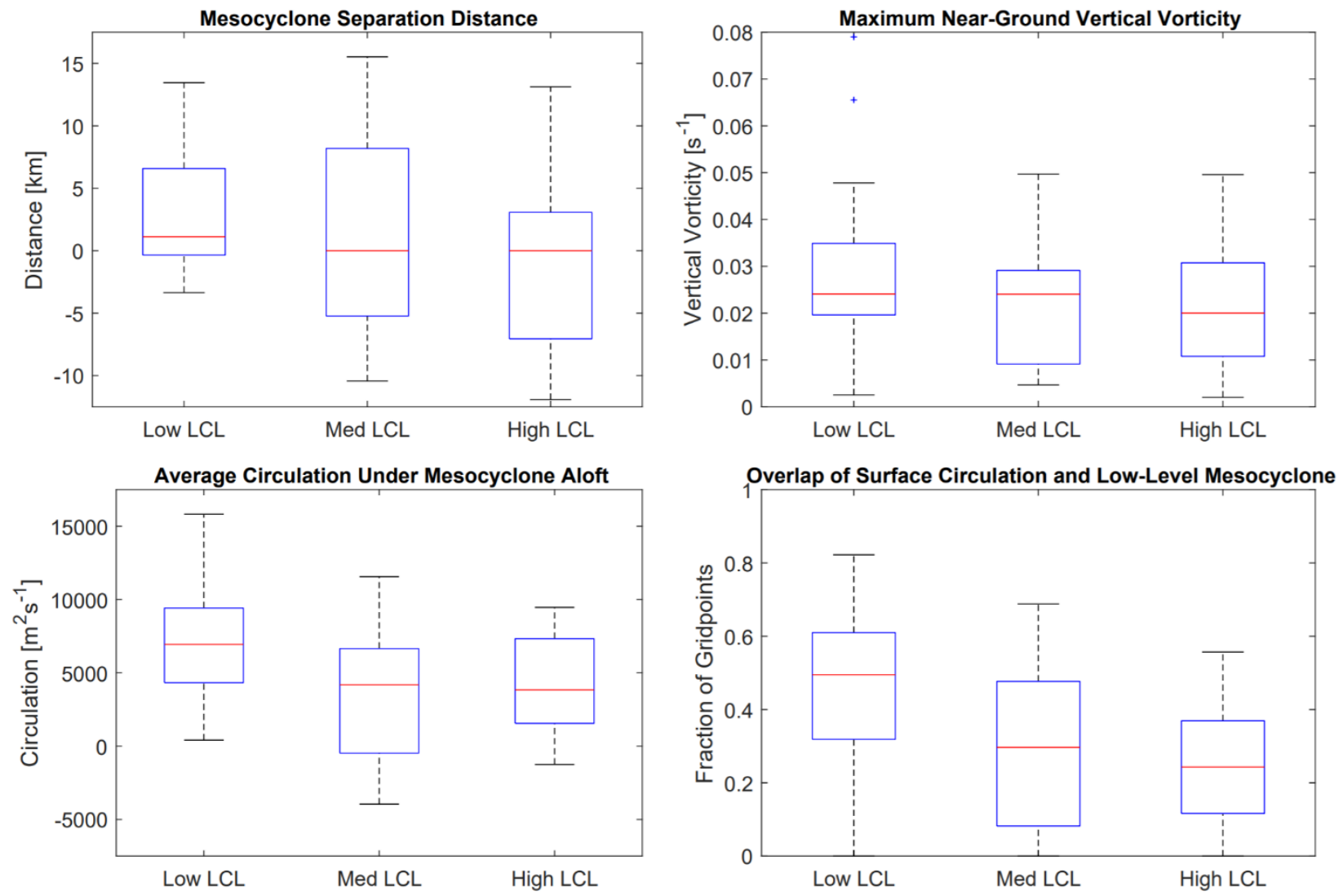

Figure 22: Same as Figure 21, but for the $\alpha=0^{\circ}$ simulations 


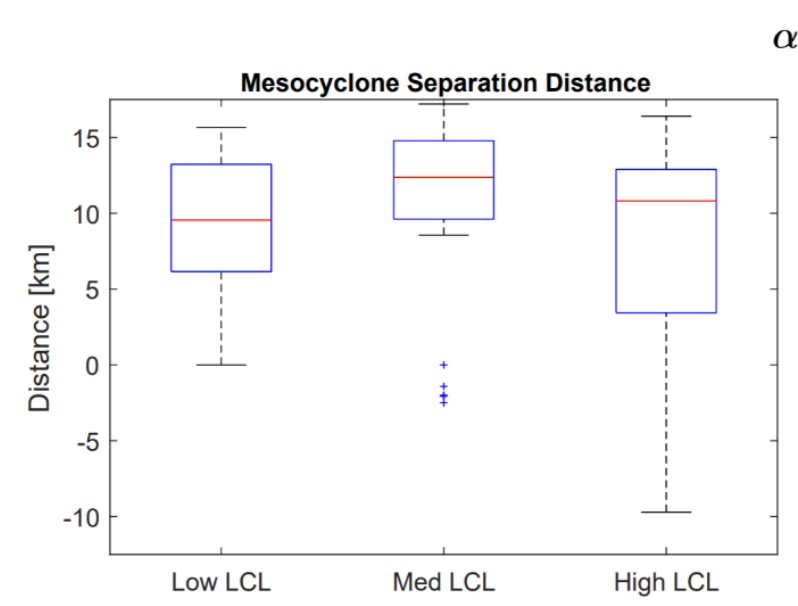

$$
\alpha=90^{\circ}
$$
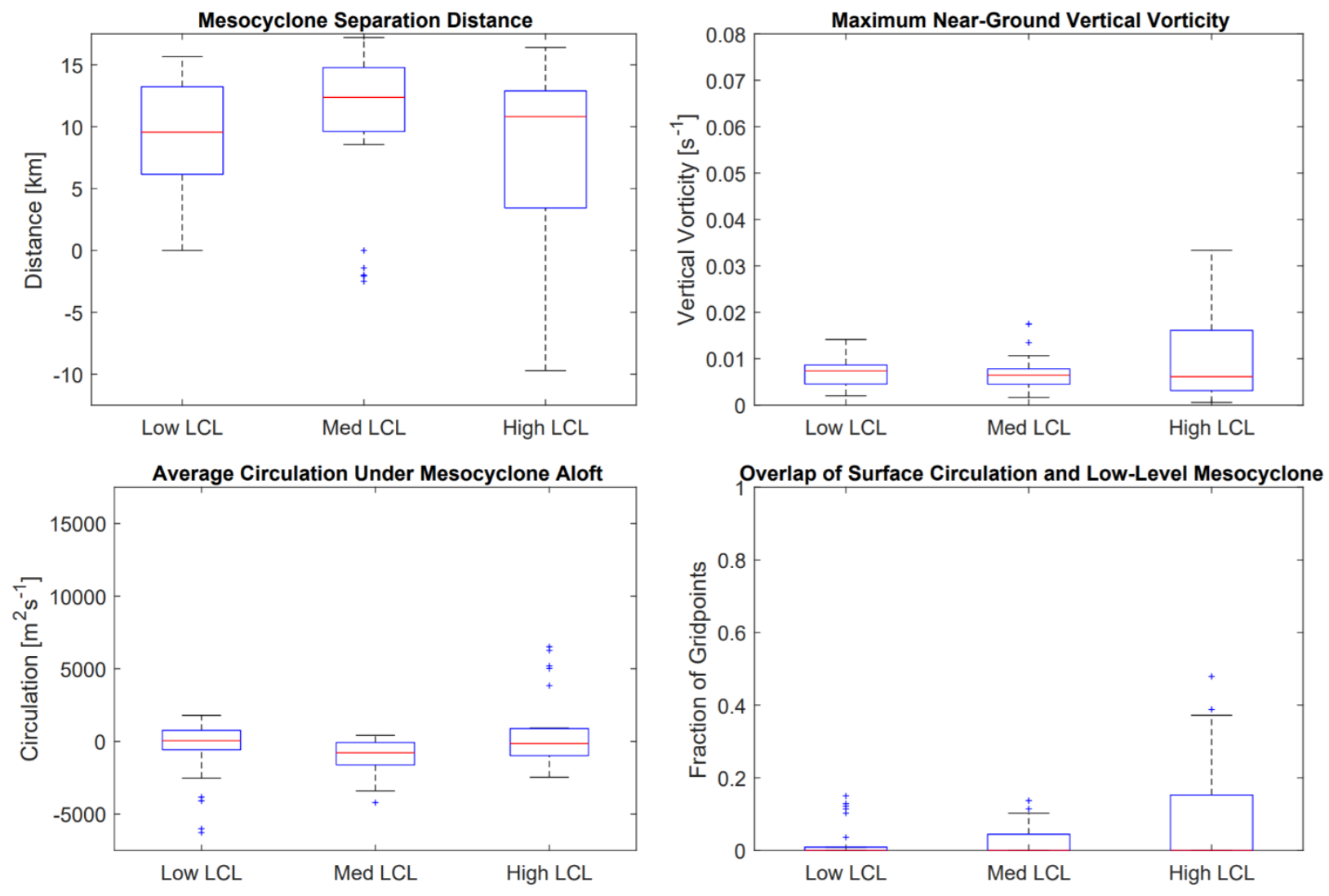

Figure 23: Same as Figure 22, but for the $\alpha=90^{\circ}$ simulations

\subsubsection{Statistical Analysis}

In order to provide more definitive statistical support for the third hypothesis, as well as address the timing issues with the presented distributions, mesocyclone separation distance at each time step during the final two hours of each simulation was correlated with near-surface vertical vorticity. G18 performed similar correlations (see G18, Figure 12) between vertical vorticity and both updraft helicity and the magnitude of separation distance. The helicity correlations were performed to determine whether the observed variability in vertical vorticity was related to differences in mesocyclone strength, rather than its vertical alignment with near- 
surface circulation. This relationship becomes more difficult to interpret in the context of this study, however, as the fact that updraft helicity is an integrated quantity (as discussed previously) leads to systematic differences in updraft helicity between LCLs (i.e., updraft helicity is integrated over a fixed layer that is defined relative to the ground, while LCL, LFC, and consequently the base of the strongest updraft vary relative to this layer between simulations). Thus the absence of a statistically significant correlation between updraft helicity and nearsurface vertical vorticity in these simulations is not surprising, for which reason these correlations are not shown.

Regarding the separation distance calculations, the magnitude of this quantity is useful in determining whether a reduction in the separation of low and mid-level circulations is correlated with surface vertical vorticity. However, it was noted earlier that vertical vorticity often remains strong even at negative separation distances (i.e., when low-level circulation has propagated ahead of the mesocyclone aloft), so using the magnitude of separation distance rather than the signed value could lessen the resultant correlation in a way that obscures the true physical relationship between these variables.

As noted by G18, the production of surface vertical vorticity does not instantaneously adjust to mesocyclone separation distance, meaning that the vertical vorticity values must be considered at some lag relative to these separation distances. A number of lag times were tested, including 0 (for means of comparison), 5, 10 and 15 minutes. Both 5 and 10-minute lags yielded similar results, but a 5-minute lag was chosen due to slightly higher correlation values. Figure 24 shows these correlations, for all the model data as well as each individual LCL. 


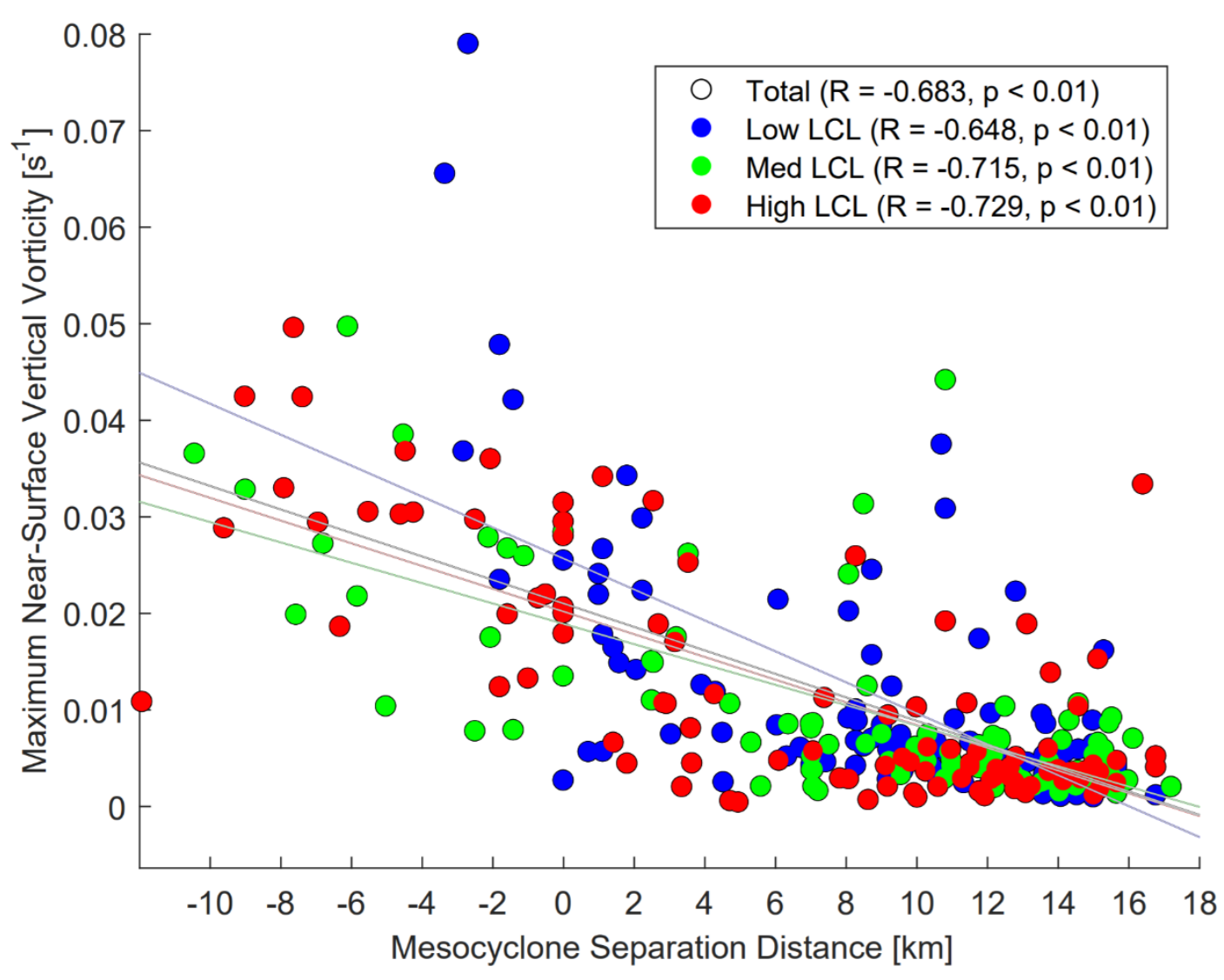

Figure 24: Scatterplot of maximum near-surface vertical vorticity (taken at 5-minute interval over the last two hours of each simulation) as a function of the magnitude of mesocyclone separation distance. The ordinate is lagged by 5 minutes from the abscissa. The linear regression line for each LCL is shown, and the legend contains correlation coefficient $(\mathrm{R})$ of each relationship, followed by its associated $\mathrm{p}$ value.

There exists a negative, statistically significant correlation between mesocyclone separation distance and vertical vorticity for each LCL, which is consistent the findings discussed thus far. Similar correlations exist when the results are separated by wind profile (not shown). The importance of this alignment can also be examined at lower levels by use of the circulation fraction, as defined in Figure 21. Figure 25 shows time series of circulation fraction for each simulation, as well as average circulation underneath the low-level mesocyclone. 


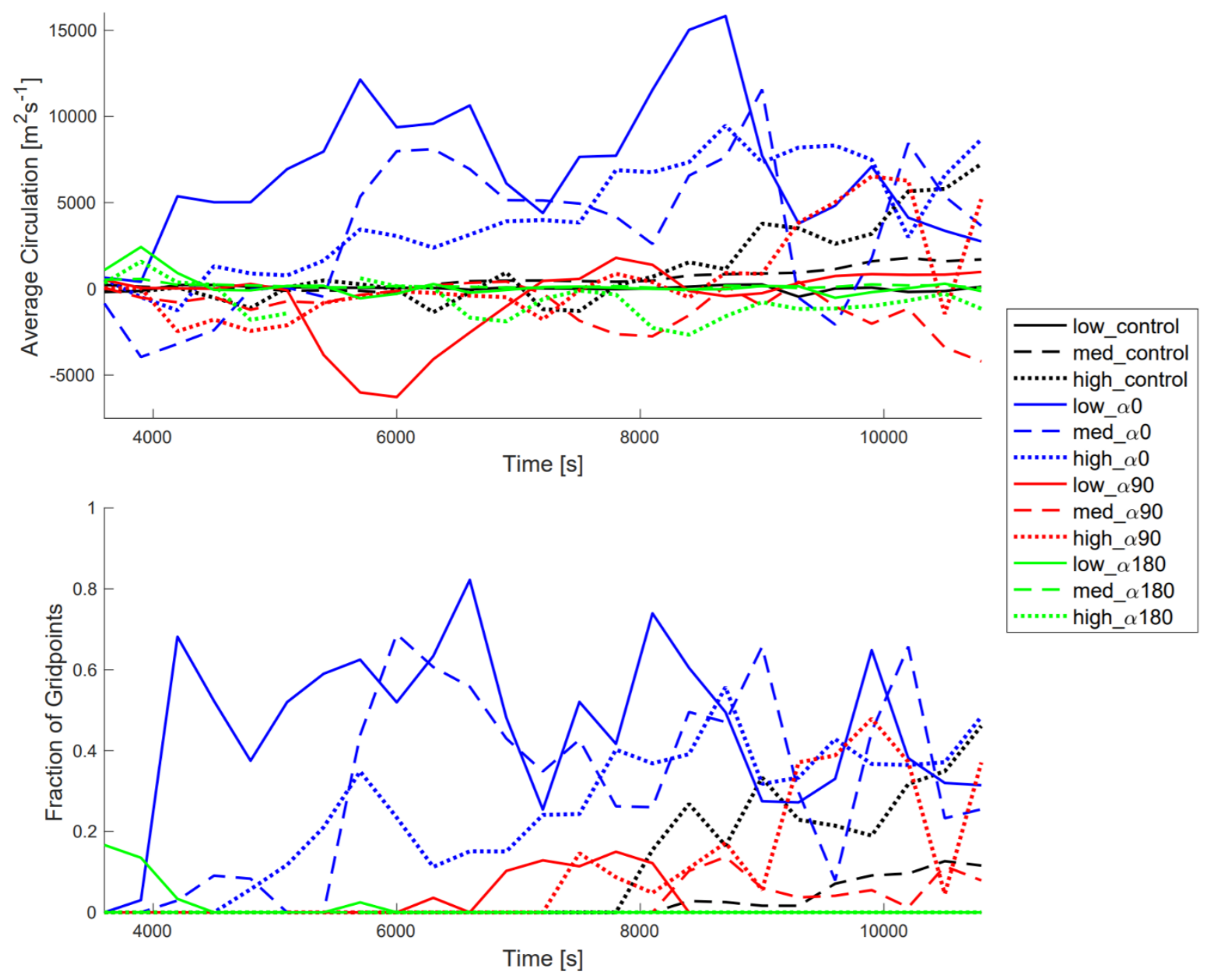

Figure 25: Time series of the average near-surface circulation (top) and fraction of gridpoints with nearsurface circulation exceeding $5000 \mathrm{~m}^{2} \mathrm{~s}^{-1}$ beneath the low-level mesocyclone (bottom), defined as the area at $\mathrm{z}=1 \mathrm{~km}$ where vertical velocity is greater than $1 \mathrm{~m} \mathrm{~s}^{-1}$ and circulation exceeds $10000 \mathrm{~m}^{2} \mathrm{~s}^{-1}$

The simulations with higher circulation located beneath the low-level mesocyclone are associated with higher values of near-surface vertical vorticity (c.f. Figure 18, Table 5).

Furthermore, increases in both average circulation and circulation fraction generally correspond with periods of decreased mesocyclone separation distance. In order to quantify this relationship, the same lag correlations in Figure 24 were performed with circulation fraction and near-surface vertical vorticity. 


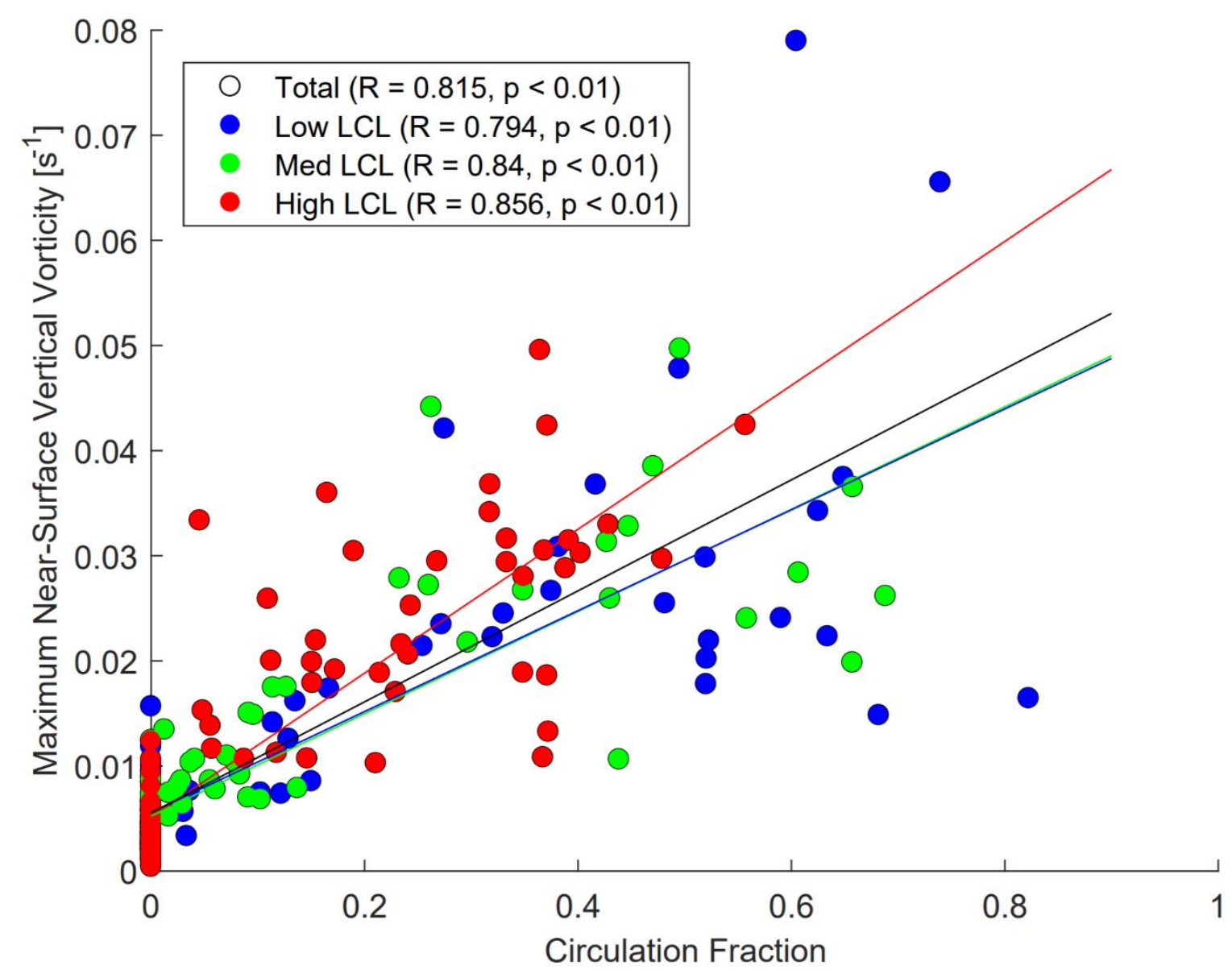

Figure 26: Same as Figure 24 (including 5-minute lag of the ordinate), but for circulation fraction as defined in Figure 21, and maximum near-surface vertical vorticity

Figure 26 shows that there is a strong positive correlation between these variables.

Furthermore, Figures 24 and 26 (and their wind profile-specific correlations, not shown) indicate that correlation improves with increasing LCL. This could indicate that at lower LCLs, positional effects are less important to the strengthening of vertical vorticity, maybe as a result of less negatively buoyant outflow that is less resistant to being dynamically lifted. By this logic, higher LCLs would require more direct alignment of the near-surface circulation and mesocyclone aloft, or at least the near-surface circulation and the low-level mesocyclone, in order to dynamically lift more negatively buoyant outflow observed in the high LCL simulations. It is worth noting 
that the large number of low vertical vorticity values occurring at a circulation fraction of zero are partially responsible for these high correlation values. This does not weaken the physical reasoning for this relationship, as the lack of overlap between the low-level mesocyclone and near-surface circulation would significantly limit any low-level upward dynamic forcing needed to intensify near-surface vertical vorticity.

The combination of the presented time series and statistical analyses have supported the first half of our third hypothesis that near-surface vertical vorticity is maximized when there is overlap between mid- and low-level circulation, or similarly when the low-level mesocyclone is positioned directly above circulation-rich air near the surface. The next section will address the second half of the hypothesis, investigating what physical mechanism is the driving force behind this relationship.

\subsection{Dynamic Influences of LCL on Vorticity Generation}

Though the thermodynamic impacts of varying LCL have been shown to influence mesocyclone separation, some dynamic mechanism must exist which bridges the relationship between separation distance and near-surface vertical vorticity. For the purpose of this study, it is hypothesized that an upward-directed VPPGF attendant to the supercell mesocyclone is largely responsible for the stretching and intensification of near-surface vertical vorticity observed when mesocyclone separation distance approaches zero. As per the methodology of a Hastings and Richardson (2016), which uses a similar approach as Rotunno and Klemp (1982, 1985), a hydrostatically balanced base state can be defined with $\overline{\theta_{p}}$ and $\bar{\pi}$ - where $\theta_{p}$ is density potential 
temperature and $\pi \equiv\left(\frac{p}{p_{0}}\right)^{R / c_{p}}$ is the Exner function - in which vertical accelerations are given by

$$
\frac{D w}{D t}=-c_{p} \overline{\theta_{p}} \frac{\partial \pi^{\prime}}{\partial z}+B
$$

where $\mathrm{w}$ is the vertical component of velocity, $c_{p}$ is the specific heat capacity at constant pressure, $\pi^{\prime}$ is the perturbation Exner function $\left(\pi^{\prime}=\pi-\bar{\pi}\right)$ which is akin to perturbation pressure, and $B$ is the acceleration due to buoyancy. The terms on the RHS of Equation 1 represent the contributions to vertical accelerations by vertical perturbation pressure gradients and buoyancy, respectively. Within the cold pool of a supercell, such as those displayed in Figures 11 and 12, the buoyancy typically provides a net negative (downward) acceleration. Therefore, it is assumed that vertical pressure gradients (specifically gradients of non-hydrostatic perturbation pressure) are primarily related to the upward acceleration which can lift near-surface circulation. However, it bears mentioning that in contrast to G18, buoyancy (in Equation 1) is changing with LCL, so this must be taken into consideration when discussing the ability for these vertical pressure gradients to lift available near-surface circulation.

The methods outlined in Hastings and Richardson (2016; Appendix A) were used to decompose the total non-dimensional perturbation pressure $\left(\pi^{\prime}\right)$ into its constituent parts - the buoyant $\left(\pi_{B}^{\prime}\right)$, linear dynamic $\left(\pi_{L D}^{\prime}\right)$, and nonlinear dynamic $\left(\pi_{N D}^{\prime}\right)$ perturbation pressures. As G18 notes, the $\pi_{B}^{\prime}$ term tends to be negative (positive) above (below) the local buoyancy minimum, $\pi_{L D}^{\prime}$ is positive (negative) upshear (downshear) of updrafts and negative (positive) upshear (downshear) of downdrafts, and $\pi_{N D}^{\prime}$ is positive in regions of deformation and negative in regions of vorticity. Though $\pi_{B}^{\prime}$ is typically positive within negatively buoyant outflow near 
the surface, its associated pressure gradient force is counteracted by the buoyancy term in Equation 1, and therefore cannot strengthen the storm updraft. Thus, the dynamic part - meaning the sum of the linear and non-linear pressure gradient forces - will be the focus of our discussion. Specifically, the dynamic VPPGF five minutes before maximum near-surface vertical vorticity will be analyzed, given the hypothesized delayed response between dynamic forcing and responses in the vorticity field (as per Figures 24 and 26). Figure 27 shows the dynamic VPPGF at $500 \mathrm{~m}$ for each LCL in the $\alpha=0^{\circ}$ and $\alpha=90^{\circ}$ cases at the time of maximum nearsurface vertical vorticity, with contours of the near-surface circulation superimposed. 

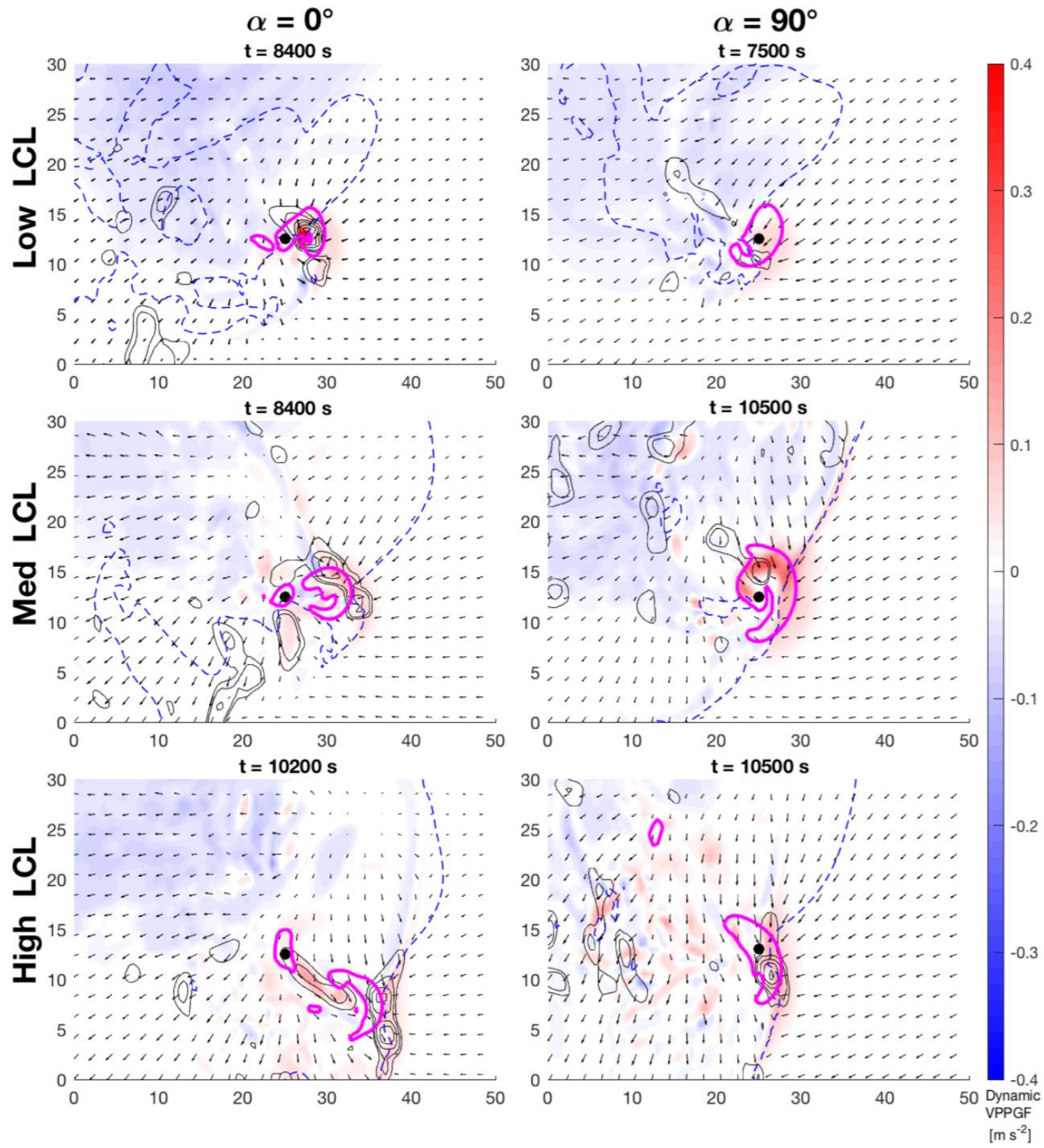

Figure 27: Plan views of dynamic vertical perturbation pressure gradient force $500 \mathrm{~m}$ AGL (shaded), circulation at the lower model surface (black contours of $0.5 \times 10^{3}, 1 \times 10^{4}, 2 \times 10^{4}, 3 \times 10^{4}, 4 \times 10^{4}, 5 \times$ $10^{4} \mathrm{~m}^{2} \mathrm{~s}^{-1}$ ), storm-relative surface winds, and the $-1 \mathrm{~K}$ surface potential temperature perturbation (dashed blue) for the $\alpha=0^{\circ}$ and $\alpha=90^{\circ}$ cases five minutes (one time step) before maximum near-surface vertical vorticity. The solid magenta line is the $500 \mathrm{~m}^{2} \mathrm{~s}^{-2}$ updraft helicity contour, and the black dot is the maximum updraft helicity. 
As expected, the mid-level mesocyclone is associated with positive low-level (500 m AGL) dynamic VPPGF leading up to the time of maximized near-surface rotation. In order to determine any trends in the decomposed VPPGFs, the average near-surface circulation and dynamic VPPGF beneath the mid-level mesocyclone were computed for each of the plots in Figure 27.

Table 6: Average near-surface circulation $\left(\mathrm{m}^{2} \mathrm{~s}^{-1}\right.$ ) and dynamic VPPGF (at $500 \mathrm{~m}$ AGL; units of $\mathrm{m} \mathrm{s}^{-2}$ ) beneath the mid-level mesocyclone (i.e., the $500 \mathrm{~m}^{2} \mathrm{~s}^{-2}$ updraft helicity contour) at the model time steps shown in Figure 23

\begin{tabular}{|c|c|c|c|c|}
\hline & \multicolumn{2}{|c|}{$\alpha=0^{\circ}$} & \multicolumn{2}{c|}{$\alpha=90^{\circ}$} \\
\hline & Circulation & Dynamic VPPGF & Circulation & Dynamic VPPGF \\
\hline Low LCL & 14214 & 0.0564 & 1600 & 0.0353 \\
\hline Medium LCL & 3675 & 0.0292 & -6182 & 0.1011 \\
\hline High LCL & 5692 & 0.0120 & 7945 & 0.0464 \\
\hline
\end{tabular}

For the $\alpha=0^{\circ}$ simulations, both the largest circulation and dynamic VPPGF beneath the mesocyclone occur in the low LCL case, with ample positive circulation and dynamic VPPGF directly beneath the mesocyclone aloft. The magnitude and collocation of these maxima likely contribute to the ample production of near-surface vertical vorticity observed in this simulation the greatest of any of the twelve model runs, let alone the $\alpha=0^{\circ}$ simulations (c.f. Table 4). Though there is still appreciable circulation and dynamic VPPGF in the other two LCL configurations, these variables are smaller in magnitude and more diffuse. Additionally, these 
fields are only partially collocated, contributing to decreased production of near-surface vertical vorticity.

For the $\alpha=90^{\circ}$ simulations, the largest dynamic VPPGF is found in the medium LCL case. However, this intense dynamic forcing is located above an area with net negative circulation, hindering the formation of intense, positive near-surface vertical vorticity. The largest circulation, though, occurs within the high LCL case, which contains substantial overlap between the circulation and dynamic VPPGF fields beneath the mid-level mesocyclone. Unsurprisingly, this simulation corresponds to the largest near-surface vertical vorticity maximum of the $\alpha=90^{\circ}$ simulations.

Interestingly enough, there does not appear to be a trend in the magnitude of dynamic VPPGF as LCL is varied in either of the presented alpha configurations. Furthermore, even large, positive dynamic VPPGF does not appear to guarantee ample production of vorticity unless it is coincident with appreciable, positive surface circulation. This provides further justification to the notion that the relative positioning of mid-level and near surface circulation is a factor in the amplification of preexisting near-surface rotation.

\subsection{Additional Considerations}

Though the focus of the study at hand is the influence of LCL on near-surface outflow and rotation, it is worth noting the sensitivity of the presented results to low-level shear orientation. As discussed previously, the LCL corresponding to the maximum near-surface vertical vorticity varies depending on the low-level shear orientation. Specifically, shear profiles with a smaller component of low-level, storm-relative winds opposing the cold pool $\left(\alpha=0^{\circ}\right)$ 
maximize their vertical vorticity with a lower LCL, while those with moderate winds opposing the cold pool $\left(\alpha=0^{\circ}, 90^{\circ}\right)$ maximize their vertical vorticity with a lower LCL. Furthermore, when there are large gust front-opposing winds, the production of near-surface vertical vorticity is limited across all LCLs. These observations, combined with information in Tables 4-5, reveal two surprising results. First is the favorability of the $\alpha=0^{\circ}$ wind profile across all three LCLs. Previous studies (i.e., Wicker 1996; Miller, 2006; Esterheld and Giuliano 2008; Nowotarski and Jensen 2013; Coffer and Parker 2017) have identified this shear profile as being unfavorable for tornadogenesis, suggesting that such a profile (with its weaker storm-relative winds opposing the gust front) results in outflow dominated storms in which low-level circulation is advected ahead of the primary midlevel updraft/mesocyclone. However, the three simulations containing the $\alpha=$ $0^{\circ}$ wind profile correspond to the three highest near-surface vorticity maxima of all the simulations performed. This result, though counterintuitive, is in agreement with the findings of G18, in which the $\alpha=0^{\circ}$ wind profile was the only low-level shear orientation which lead to the formation of intense near-surface vertical vorticity. Similar to the reasoning presented by G18, it appears that decreased gust front-opposing winds in this case actually facilitates a rapid decrease of mesocyclone separation distance, allowing for maximum dynamic low-level updraft forcing and stretching of near-surface circulation. Though this often leads to increasingly negative mesocyclone distances later in these simulations, near-surface vertical vorticity appears to remain high even when it has surged well ahead of the updraft core, which is consistent with the results of Dowell and Bluestein (2002) as discussed in Section 4.3.2.

The second surprising result is that low_ $\alpha 90$ did not contain the largest vertical vorticity values of the $\alpha=90^{\circ}$ simulations. Given the storm climatologies discussed, it would be expected that this environment would be prime for the formation of intense near-surface vertical vorticity, 
given its low LCL and ample streamwise vorticity associated with its hodograph shape. However, low_ $\alpha 90$ corresponds to the lowest near-surface vertical vorticity of the $\alpha=90^{\circ}$ simulations, with high_ $\alpha 90$ producing near-surface vertical vorticity over twice as strong. It appears that the reason for this disparity once again lies in the alignment of the low-level circulation with the mesocyclone aloft, which in this case is related to more negative outflow buoyancy associated with the high LCL configuration. Whereas low_ $\alpha 90$ contains a brief and moderate collapse of mesocyclone separation distance roughly halfway through the simulation, high_ $\alpha 90$ shows a sharper and more sustained drop in separation distance, as well as greater overlap between the low-level mesocyclone and near-surface circulation.

At face value, these unexpected findings could bring into question the physical relevance of the results presented. It is therefore important to remind the reader that given the constraints of using a numerical model, the intent of this study is not to identify specific combinations of LCL and low-level shear orientation which favor tornadogenesis. Rather, its goal is to examine the sensitivity of the storm morphology and maximum near-surface vertical vorticity to these parameters, so as to highlight the basic physical mechanisms that might contribute to the development of near-surface vertical vorticity. In reality, the existence of heterogeneity in the near-storm environment, convergent outflow, and complex microphysical processes, among other things add a layer of complexity to the process of tornadogenesis which extend beyond the capabilities of our storm-scale numerical models, at least for the time being.

Since this study and G18 have both conclude that minimizing the displacement between mid-level and near-surface circulation is crucial to the intensification of vertical vorticity at the surface, one might ask - which is more important, low-level shear or LCL (and its contribution to outflow buoyancy), in determining this mesocyclone separation? Though the answer to this 
question likely depends on the dynamic and thermodynamic base state of the analyzed simulations, some basic statistics can be implemented to address this matter. In order to perform these statistics, indicators of mesocyclone separation distance, LCL influence on outflow buoyancy, and low-level shear orientation were implemented in order to broadly characterize each simulation. These distances are replaced by the spread in mesocyclone distance, meaning the difference between the maximum distance prior to the time of the near-surface vorticity maximum and the minimum distance afterwards. Thus, this metric communicates the trend in mesocyclone distance without being inaccurately influenced by inherent variability associated with the methodology used in computing mesocyclone separation distance. Outflow buoyancy is approximated using the average cold pool buoyancy during the final 90 minutes of each simulation (as per Figure 14), and gust-front opposing winds are taken from Table 7.

Correlating just the mesocyclone separation distance spread and these gust-front opposing winds results in a weak relationship with and $R$ and $R^{2}$ values of -0.33 and 0.11 , respectively. This implies that only $11 \%$ percent of the variance seen in the distance spread can be explained by changes in gust-front opposing winds. If a multivariate linear regression is performed with all three quantities, however, the $\mathrm{R}$ and adjusted $\mathrm{R}^{2}$ (necessary for comparison to standard regression) values jump to 0.85 and 0.67 , respectively. This implies that outflow buoyancy accounts for five times the variance in the distance spread explained by gust frontopposing winds alone. Furthermore, this suggests that information regarding outflow buoyancy and these gust front-opposing winds can explain two thirds of the variance seen in the mesocyclone separation spread. These calculations, though limited in their scope, underscore the relative importance of LCL and its effect on outflow buoyancy for the strengthening of nearsurface vertical vorticity, as well as its crucial interactions with the low-level wind profile. 


\section{SUMMARY AND CONCLUSIONS}

In this study, the impact of varying LCL on outflow characteristics and the production of near-surface vertical vorticity was tested through a set of idealized supercell simulations. In general, it was hypothesized that changing LCL within these simulations would lead to differences in outflow buoyancy, which in turn would affect the relative positioning of the nearsurface circulation and mesocyclone aloft, which provides the dynamic updraft forcing required for intensification of near-surface rotation. Now that the performed model simulations have been thoroughly analyzed, let us explicitly revisit and assess the validity of each of the hypotheses set forth in Section 2.4.

1. Changes in LCL will affect cold pool buoyancy in supercell thunderstorms, with higher (lower) LCLs leading to more (less) negatively buoyant outflow.

There is a distinct tendency towards more negatively buoyant cold pools as LCL is increased, particularly later in the model runs when the precipitation structure and resultant outflow have fully developed. Given similar accumulated rainfall values across the three LCL configurations, as well as the decreasing of sub-cloud relative humidity with increasing LCL, it is likely that these observed variations in outflow buoyancy are related to increased evaporation of rainfall in environments with higher LCLs.

2. A lower LCL will lead to less forward propagation of outflow and embedded nearsurface circulation relative to the mesocyclone aloft in supercells, compared to higher LCLs.

Outflow propagation in the simulations can be compared using a number of different metrics - the first zero-crossing of mesocyclone separation distance occurring later in 
simulations (relative to higher LCL simulations), the rate of change of these distances, or less negative values of separation distance altogether (i.e., the low level circulation does not propagate as far forward as the mesocyclone aloft), among others. Section 4.3.2 addresses all of these metrics, which consistently indicate that higher LCLs lead to more forward propagation of outflow - with these LCL configurations corresponding to increasingly negative linear of trend of the mesocyclone separation distances (and thus earlier zero-crossings of this distance), as well as more negative values of separation distance relative to lower LCLs. Though the distinction between medium and high LCLs is less clear, these factors indicate that as LCL is increased, near-surface circulation generally propagates faster and is more likely to advect beneath and subsequently downwind of the mesocyclone aloft.

3. Near-surface vertical vorticity will be largest when the horizontal distance between the near-surface circulation and the mesocyclone aloft is minimized and the dynamic vertical perturbation pressure gradient force coincident with near-surface circulation is maximized.

The first part of this final hypothesis has been demonstrated both in this study, and by G18. The time series presented in Figure 18, as well as the correlations and analysis in Figures 20-22 demonstrate that decreased distance between the near-surface circulation and mesocyclone aloft, or similarly increased overlap between the low-level mesocyclone and preexisting near-surface rotation, subsequently leads to an intensification of nearsurface vertical vorticity.

The relationship described in the second portion was validated by decomposing the simulated pressure fields and analyzing the strength and positioning of the dynamic 
VPPGF within the storms (as shown in Figure 27 and Table 6). In the analyzed simulations, maximum near-surface vertical vorticity for a given LCL and shear orientation is associated with a positive dynamic VPPGF located beneath the mid-level mesocyclone. However, there is not a direct relationship between dynamic VPPGF and near-surface vertical vorticity, meaning that maximum dynamic VPPGF does not guarantee the maximum production of vertical vorticity. The aforementioned positive dynamic VPPGF cannot fully contribute to the strengthening of near-surface vertical vorticity until it is collocated with appreciable, positive circulation at the surface, which supports the second half of the third hypothesis.

There is substantial future work that is necessary to fully understand the influence of LCL on supercell evolution and the formation of intense near-surface rotation. Possible research topics worth investigating include:

1. Confirming the findings of this study, as well as those of G18, by analyzing the near storm environments of observed tornadic supercell cases, particularly those with higher LCLs and/or an ambient wind profile similar to our $\alpha=0^{\circ}$ simulations

As described throughout Section 4.5, not all of the findings of this study fall in line with existing literature or observations of tornadic supercells - low LCL and an ambient shear profile with ample streamwise vorticity - given storm climatologies within the literature (ie. Rasmussen and Blanchard 1998; Thompson et al. 2003; Craven and Brooks 2004; Esterheld and Giuliano 2008). Thus, it would be of interest to determine whether the storm environments which produced intense near-surface vertical vorticity are found 
within nature. If not, then some other physical mechanism must be examined in order to explain the divergence of our simulation results from realistic storm characteristics. Tangentially, a case study analysis could help confirm or refute the physical relevance of mesocyclone separation distance as it relates to the strengthening of near-surface vertical vorticity.

2. Examine the impact of LCL height relative to the shear layer, and its potential effect on precipitation characteristics in supercell simulations

One unexplored consequence of changing LCL in the manner prescribed within this study is are slight changes in the height of LCL relative to its shear profile. Though it is unclear whether this would noticeably influence the characteristics of the simulated storms, one can imagine that this could affect the spatial distribution and size sorting of hydrometers condensing near the LCL. Such alterations could thus influence the observed patterns in accumulated surface precipitation, and by extension the buoyancy and orientation of storm outflow. These changes would likely be minor, but performing additional sensitivity tests such as this would help establish the validity and/or any caveats to this LCL alteration method.

3. Decompose the vorticity field into contributions from separate terms in the vertical vorticity equation to understand the origins of rotation within the simulated storms, and their sensitivity to varying $L C L$

As described earlier in this study, vorticity can be generated in a number of ways within a supercell, such as baroclinically along the rear gust front or in the precipitation region, or through the reorientation and amplification of barotropic and baroclinic vorticity. By 
decomposing the vorticity field by terms in the vorticity equation (tilting, baroclinic, etc.), one can determine how vorticity is being generated and deformed within the storm environment. Furthermore, comparing the decomposed fields across the tested LCL configurations tested could shed light on whether changing low-level moisture affects the magnitude and relative contribution of these components to the total vorticity field.

Overall, this study has provided intriguing insight into the impact of varying low-level moisture on outflow and rotation within idealized supercell thunderstorms. Though there is not one particular LCL which always favors the development of intense near-surface rotation, it is clear that LCL has an influence on the outflow characteristics in simulated supercells. These outflow characteristics, in combination with the low-level wind profile, dictate the relative positioning of mid-level and near-surface circulation, which in turn regulates the production of near-surface vertical vorticity. Thus, LCL can be said to play a nuanced, yet crucial role in determining whether intense surface rotation is able to form within simulated supercells. 


\section{REFERENCES}

Adlerman, E. J., \& Droegemeier, K. K. (2002). The sensitivity of numerically simulated cyclic mesocyclogenesis to variations in model physical and computational parameters. Monthly weather review, 130(11), 2671-2691.

Adlerman, E. J., Droegemeier, K. K., \& Davies-Jones, R. (1999). A numerical simulation of cyclic mesocyclogenesis. Journal of the atmospheric sciences, 56(13), 2045-2069.

Brandes, E. A. (1978). Mesocyclone evolution and tornadogenesis: Some observations. Monthly Weather Review, 106(7), 995-1011.

Brooks, H. E., Doswell III, C. A., \& Cooper, J. (1994). On the environments of tornadic and nontornadic mesocyclones. Weather and forecasting, 9(4), 606-618.

Browning, K. A. (1964). Airflow and precipitation trajectories within severe local storms which travel to the right of the winds. Journal of the Atmospheric Sciences, 21(6), 634-639.

Bryan, G. H., 2014: The governing equations for $\mathrm{cm} 1$. [Available online at http:

//www2.mmm.ucar.edu/people/bryan/cm1/].

Bryan, G. H., \& Fritsch, J. M. (2002). A benchmark simulation for moist nonhydrostatic numerical models. Monthly Weather Review, 130(12), 2917-2928.

Coffer, B. E., \& Parker, M. D. (2015). Impacts of increasing low-level shear on supercells during the early evening transition. Monthly Weather Review, 143(5), 1945-1969.

Coffer, B. E., \& Parker, M. D. (2017). Simulated supercells in nontornadic and tornadic VORTEX2 environments. Monthly Weather Review, 145(1), 149-180. 
Craven, J. P., Brooks, H. E., \& Hart, J. A. (2004). Baseline climatology of sounding derived parameters associated with deep, moist convection. Natl. Wea. Dig, 28(1), 13-24.

Davies-Jones, R. P. (1982). Observational and theoretical aspects of tornadogenesis. In Intense Atmospheric Vortices (pp. 175-189). Springer, Berlin, Heidelberg.

Davies-Jones, R. (1984). Streamwise vorticity: The origin of updraft rotation in supercell storms. Journal of the atmospheric sciences, 41(20), 2991-3006.

Davies-Jones, R., 2006: Tornadogenesis in supercells: what we know and what we don't know. Preprints, 23rd Conf. on Severe Local Storms, San Antonio, TX, Amer. Meteor. Soc.

Davies-Jones, R.P., Brooks, H.E., 1993. Mesocyclogenesis from a theoretical perspective. Tornadoes and tornadic storms: a review of conceptual models. The Tornado: Its Structure, Dynamics, Prediction, and Hazards. Geophys. Monogr. Amer. Geophys. Union, pp. 105-114. No. 79.

Davies-Jones, R.P., Trapp, R.J., Bluestein, H.B., 2001. Tornadoes and tornadic storms. Meteor. Monogr. Amer. Meteor. Soc., pp. 167-221. No. 50.

Deardorff, J. W. (1980). Stratocumulus-capped mixed layers derived from a three-dimensional model. Boundary-Layer Meteorology, 18(4), 495-527.

Dowell, D. C., \& Bluestein, H. B. (2002). The 8 June 1995 McLean, Texas, storm. Part I: Observations of cyclic tornadogenesis. Monthly weather review, 130(11), 2626-2648.

Durran, D. R., \& Klemp, J. B. (1983). A compressible model for the simulation of moist mountain waves. Monthly Weather Review, 111(12), 2341-2361. 
Dutton, J. A., \& Johnson, D. R. (1967). The theory of available potential energy and a variational approach to atmospheric energetics. In Advances in geophysics (Vol. 12, pp. 333-436). Elsevier.

Esterheld, J. M., \& Giuliano, D. J. (2008). Discriminating between tornadic and non-tornadic supercells: A new hodograph technique. E-Journal of Severe Storms Meteorology, 3(2).

Frame, J., \& Markowski, P. (2013). Dynamical influences of anvil shading on simulated supercell thunderstorms. Monthly Weather Review, 141(8), 2802-2820.

Gilmore, M. S., \& Wicker, L. J. (1998). The influence of midtropospheric dryness on supercell morphology and evolution. Monthly weather review, 126(4), 943-958.

Grasso, L. D., \& Cotton, W. R. (1995). Numerical simulation of a tornado vortex. Journal of the atmospheric sciences, 52(8), 1192-1203.

Grzych, M. L., Lee, B. D., \& Finley, C. A. (2007). Thermodynamic analysis of supercell rearflank downdrafts from Project ANSWERS. Monthly weather review, 135(1), 240-246.

Guarriello, F., Nowotarski, C. J., \& Epifanio, C. C. (2018). Effects of the Low-Level Wind Profile on Outflow Position and Near-Surface Vertical Vorticity in Simulated Supercell Thunderstorms. Journal of the Atmospheric Sciences, 75(3), 731-753.

Hastings, R., \& Richardson, Y. (2016). Long-term morphological changes in simulated supercells following mergers with nascent supercells in directionally varying shear. Monthly Weather Review, 144(2), 471-499.

James, R. P., Markowski, P. M., \& Fritsch, J. M. (2006). Bow echo sensitivity to ambient moisture and cold pool strength. Monthly weather review, 134(3), 950-964. 
Johns, R. H., \& Doswell III, C. A. (1992). Severe local storms forecasting. Weather and Forecasting, 7(4), 588-612.

Johns, R. H., Davies, J. M., \& Leftwich, P. W. (1993). Some wind and instability parameters associated with strong and violent tornadoes: 2. Variations in the combinations of wind and instability parameters. The Tornado: Its Structure, Dynamics, Prediction, and Hazards, 583-590.

Klemp, J. B., \& Rotunno, R. (1983). A study of the tornadic region within a supercell thunderstorm. Journal of the Atmospheric Sciences, 40(2), 359-377.

Klemp, J. B., \& Wilhelmson, R. B. (1978). The simulation of three-dimensional convective storm dynamics. Journal of the Atmospheric Sciences, 35(6), 1070-1096.

Kosiba, K., Wurman, J., Richardson, Y., Markowski, P., Robinson, P., \& Marquis, J. (2013). Genesis of the Goshen County, Wyoming, tornado on 5 June 2009 during VORTEX2. Monthly Weather Review, 141(4), 1157-1181.

Lemon, L. R., and C. A. Doswell, 1979: Severe thunderstorm evolution and mesocyclone structure as related to tornadogenesis. Mon. Wea. Rev., 107, 1184-1197.

Lerach, D. G., \& Cotton, W. R. (2012). Comparing aerosol and low-level moisture influences on supercell tornadogenesis: Three-dimensional idealized simulations. Journal of the Atmospheric Sciences, 69(3), 969-987.

Lilly, D. K. (1979). The dynamical structure and evolution of thunderstorms and squall lines. Annual Review of Earth and Planetary Sciences, 7(1), 117-161.

Lilly, D. K. (1982). The development and maintenance of rotation in convective storms. In Intense Atmospheric Vortices(pp. 149-160). Springer, Berlin, Heidelberg. 
Mansell, E. R., Ziegler, C. L., \& Bruning, E. C. (2010). Simulated electrification of a small thunderstorm with two-moment bulk microphysics. Journal of the Atmospheric Sciences, 67(1), 171-194.

Markowski, P. M. (2002). Hook echoes and rear-flank downdrafts: A review. Monthly Weather Review, 130(4), 852-876.

Markowski, Paul. "Markowski Research Group - in the Department of Meteorology \& Atmospheric Science at Penn State University.” Markowski Research Group, Penn State University, 2018, sites.psu.edu/pmarkowski/.

Markowski, P., Richardson, Y., Rasmussen, E., Straka, J., Davies-Jones, R., \& Trapp, R. J. (2008). Vortex lines within low-level mesocyclones obtained from pseudo-dual-Doppler radar observations. Monthly Weather Review, 136(9), 3513-3535.

Markowski, P. M., \& Richardson, Y. P. (2009). Tornadogenesis: Our current understanding, forecasting considerations, and questions to guide future research. Atmospheric Research, 93(13), 3-10.

Markowski, P., \& Richardson, Y. (2011). Mesoscale meteorology in midlatitudes (Vol. 2). John Wiley \& Sons.

Markowski, P. M., \& Richardson, Y. P. (2014). The influence of environmental low-level shear and cold pools on tornadogenesis: Insights from idealized simulations. Journal of the Atmospheric Sciences, 71(1), 243-275. 
Marquis, J., Richardson, Y., Markowski, P., Dowell, D., \& Wurman, J. (2012). Tornado maintenance investigated with high-resolution dual-Doppler and EnKF analysis. Monthly Weather Review, 140(1), 3-27.

McCaul Jr, E. W., \& Cohen, C. (2002). The impact on simulated storm structure and intensity of variations in the mixed layer and moist layer depths. Monthly weather review, 130(7), 17221748.

Miller, D. J. (2006, November). Observations of low level thermodynamic and wind shear profiles on significant tornado days. In Preprints, 23rd Conf. on Severe Local Storms, St. Louis, MO, Amer. Meteor. Soc (Vol. 3).

Moller, A. R., Doswell III, C. A., Foster, M. P., \& Woodall, G. R. (1994). The operational recognition of supercell thunderstorm environments and storm structures. Weather and Forecasting, 9(3), 327-347.

Morrison, H., Curry, J. A., \& Khvorostyanov, V. I. (2005). A new double-moment microphysics parameterization for application in cloud and climate models. Part I: Description. Journal of the Atmospheric Sciences, 62(6), 1665-1677.

NOAA, National Severe Storms Laboratory, “Severe Weather 101.”, 2018, www.nssl.noaa.gov/education/svrwx101/.

Nowotarski, C. J., \& Jensen, A. A. (2013). Classifying proximity soundings with self-organizing maps toward improving supercell and tornado forecasting. Weather and Forecasting, 28(3), 783801. 
Rasmussen, E. N., \& Blanchard, D. O. (1998). A baseline climatology of sounding-derived supercell andtornado forecast parameters. Weather and Forecasting, 13(4), 1148-1164.

Rogers, R. R., and M. Yau, 1989: A short course in cloud physics, International series in natural philosophy. 3rd ed., Elsevier, Burlington, MA.

Rotunno, R. (1981). On the evolution of thunderstorm rotation. Monthly Weather Review, 109(3), 577-586.

Rotunno, R., \& Klemp, J. B. (1982). The influence of the shear-induced pressure gradient on thunderstorm motion. Monthly Weather Review, 110(2), 136-151.

Rotunno, R., \& Klemp, J. (1985). On the rotation and propagation of simulated supercell thunderstorms. Journal of the Atmospheric Sciences, 42(3), 271-292.

Rotunno, R., Klemp, J. B., \& Weisman, M. L. (1988). A theory for strong, long-lived squall lines. Journal of the Atmospheric Sciences, 45(3), 463-485.

Shabbott, C.J., Markowski, P.M., 2006. Surface in situ observations within the outflow of forward-flank downdrafts of supercell thunderstorms. Mon. Weather Rev. 134, 1422-1441

Sherburn, K. D., \& Parker, M. D. (2014). Climatology and ingredients of significant severe convection in high-shear, low-CAPE environments. Weather and forecasting, 29(4), 854-877.

Snook, N., \& Xue, M. (2008). Effects of microphysical drop size distribution on tornadogenesis in supercell thunderstorms. Geophysical Research Letters, 35(24).

Straka, J. M., E. N. Rasmussen, R. P. Davies-Jones, and P. M. Markowski, 2007: An observational and idealized numerical examination of low-level counter-rotating vortices toward the rear flank of supercells. Electron. J. Severe Storms Meteor., 2, 1-22. 
Thompson, R. L., \& Edwards, R. (2000). An overview of environmental conditions and forecast implications of the 3 May 1999 tornado outbreak. Weather and forecasting, 15(6), 682-699.

Thompson, R. L., Edwards, R., Hart, J. A., Elmore, K. L., \& Markowski, P. (2003). Close proximity soundings within supercell environments obtained from the Rapid Update Cycle. Weather and Forecasting, 18(6), 1243-1261.

Thorpe, A. J., Miller, M. J., \& Moncrieff, M. W. (1982). Two-dimensional convection in nonconstant shear: A model of mid-latitude squall lines. Quarterly Journal of The Royal Meteorological Society, 108(458), 739-762.

Van Den Heever, S. C., \& Cotton, W. R. (2004). The impact of hail size on simulated supercell storms. Journal of the atmospheric sciences, 61(13), 1596-1609.

Weisman, M. L., \& Klemp, J. B. (1982). The dependence of numerically simulated convective storms on vertical wind shear and buoyancy. Monthly Weather Review, 110(6), 504-520.

Weisman, M. L., \& Klemp, J. B. (1984). The structure and classification of numerically simulated convective stormsin directionally varying wind shears. Monthly Weather Review, 112(12), 2479-2498.

Weisman, M. L., \& Klemp, J. B. (1986). Characteristics of isolated convective storms. In Mesoscale meteorology and forecasting (pp. 331-358). American Meteorological Society, Boston, MA.

Wicker, L. J. (1996, February). The role of near surface wind shear on low-level mesocyclone generation and tornadoes. In Preprints, 18th Conf. on Severe Local Storms, San Francisco, CA, Amer. Meteor. Soc (Vol. 115). 
Xue, M. (2000). Density currents in two-layer shear flows. Quarterly Journal of the Royal Meteorological Society, 126(565), 1301-1320. 


\title{
APPENDIX
}

\section{Surface Pressure Sensitivity Testing}

\author{
Methodology
}

As addressed earlier, the process implemented to change LCL height within the model input soundings results in varying surface pressures. In order to draw distinct conclusions about the impacts of varying LCL height on the resultant storms, it must be determined what secondary effect, if any, these pressure differences may have on storm structure and outflow characteristics. To this end, a series of sensitivity tests were developed to isolate and identify surface pressure effects within the simulated storms. Similar to the LCL height method, a base sounding is chosen containing a Weisman-Klemp thermodynamic profile (Weisman and Klemp 1982) and a surface pressure of $1000 \mathrm{mb}$. This sounding is then transformed using the same non-dimensional pressure calculation method implemented within $\mathrm{CM} 1$ to create two new soundings with identical temperature and relative humidity profiles as the base sounding, but with differing surface pressures of $950 \mathrm{mb}$ and $1050 \mathrm{mb}$ to capture the magnitude of surface pressure variability within the original model input soundings. The outcome of this process is three soundings that are - for all intents and purposes - thermodynamically and dynamically comparable, meaning that any differences between simulations initialized with these soundings must be driven by the surface pressure differences. Table 7 contains information about these pressure soundings.

Table 7: Same as Table 2, but for the three Weisman-Klemp pressure soundings

\begin{tabular}{|c|c|c|c|c|c|c|}
\hline & CAPE & ML CAPE & MU CAPE & CIN & ML CIN & MU CIN \\
\hline $\mathbf{9 5 0} \mathbf{~ m b}$ & 2249 & 2253 & 2929 & -51 & -20 & 0 \\
\hline $\mathbf{1 0 0 0} \mathbf{~ m b}$ & 2091 & 2380 & 2758 & -49 & -21 & 0 \\
\hline $\mathbf{1 0 5 0} \mathbf{~ m b}$ & 1923 & 2193 & 2590 & -47 & -21 & 0 \\
\hline
\end{tabular}




\begin{tabular}{|r|c|c|c|c|c|c|}
\hline AVERAGE & 2087.7 & 2275.3 & 2759 & & & \\
\hline RANGE & 326 & 187 & 339 & & & \\
\hline
\end{tabular}

\begin{tabular}{|r|c|c|c|}
\hline & LCL Height & LFC Height & EL Height \\
\hline $\mathbf{9 5 0} \mathbf{~ m b}$ & 1007 & 1600 & 12100 \\
\hline $\mathbf{1 0 0 0} \mathbf{~ m b}$ & 1006 & 1600 & 11930 \\
\hline $\mathbf{1 0 5 0} \mathbf{~ m b}$ & 1006 & 1600 & 11650 \\
\hline
\end{tabular}

The differences in CAPE across the pressure soundings exceed the $10 \%$ criteria established earlier for surface-based and most unstable parcels, but it will be shown that even differences of these magnitude have minimal effect on the resulting storm structure and characteristics. The remainder of the presented variables, namely CIN and LCL height, are nearly identical, further proving the thermodynamic similarity of the three soundings.

In order to diagnose potential effects of varying surface pressure on storm structure, a set of three CM1 simulations is designed with these surface pressure soundings and a quarter-circle hodograph with $\alpha=90^{\circ}$ low-level shear orientation. These simulations are run with the same specifications as described in Table 1, and integrated over the same three hour period, and their results are analyzed the same way as the original CM1 runs. These results are evaluated both individually to assess their physical plausibility, as well in relation to one another. It is hypothesized that if the initiation and time evolution of the storms in each simulation are similar, then it can be concluded that simply changing surface pressure does not have any first-order dynamic effects on the storm structure. 
Though the CM1 simulations show that the surface pressure changes have a minimal dynamic effect on resulting supercells, the thermodynamic motivation for this study necessitates additional sensitivity testing. In particular, it is important to verify that these surface pressure changes do not affect the strength, depth, and propagation of storm outflow, all of which influence the storm characteristics analyzed in the proceeding sections. These properties are examined by constructing a set of 2-D, "dam-break style” cold pool simulations using CM1, initialized with the three surface pressure soundings. Two wind profiles $-\alpha=0^{\circ}$ and $\alpha=90^{\circ}-$ are applied such that the tested low-level shear orientations that are both orthogonal and perpendicular to the cold pool, respectively. These simulations are run with the following specifications.

Table 8: Table of model specifications for cold pool simulations

\begin{tabular}{|c|c|}
\hline Horizontal Grid Space & $500 \mathrm{~m}$ \\
\hline Vertical Grid Space & $500 \mathrm{~m}$ \\
\hline Horizontal Grid Points & 200 \\
\hline Vertical Grid Points & 20 \\
\hline Cold Pool Depth & $2500 \mathrm{~m}$ \\
\hline Maximum Theta Perturbation & $-6 \mathrm{~K}$ \\
\hline
\end{tabular}

Each simulation is integrated for two hours, and analyzed to determine the depth, speed, and intensity of the resultant density current. As with the CM1 simulations initialized with these pressure soundings, the notion is that for a given low-level shear orientation, if cold pool characteristics are comparable across the three simulations then it can be concluded that surface pressure does not have a distinct impact on outflow characteristics and propagation. If they are 
not comparable, then the differences between the simulations will be identified and taken into consideration during our discussion of outflow propagation in later sections.

\section{Storm Structure Results}

The results of the surface pressure sensitivity tests were analyzed in order to establish the validity and implications of our LCL alteration method. Figure 28 shows the reflectivity structure for the 950-, 1000-, and 1050-mb surface pressure simulations (denoted as wk_950mb, wk_1000mb, wk_1050mb, respectively; wk referring to the Weisman-Klemp thermodynamic profile used to construct each sounding) at hours one, two, and three of model integration.

Comparing the output from each simulation with varying surface pressure, the evolution and morphology of each run are similar. After one hour of integration, each storm takes on supercellular characteristics, including the presence of both a sustained updraft collocated with a coherent mid-level mesocyclone, as well as the beginning of the formation of a cold pool. Two hours into the model run, these storms mature into classic supercells with well-defined hook echoes. Each simulation develops a broad cold pool extending from the northeast to southwest of the updraft helicity maximum. By the last integration step, all three storms continue to maintain the same structure, with a slightly weakened reflectivity signature, and more precipitation in the vicinity of inflow notch. The surface vertical vorticity maxima shift from eastward beneath the mid-level mesocyclone in both the 950 and 1050-mb simulations. The 1000-mb simulation also develops vertical vorticity of the same magnitude beneath the mid-level mesocyclone at this time, however, a second stronger vorticity maximum exists west of the mesocyclone as well. 

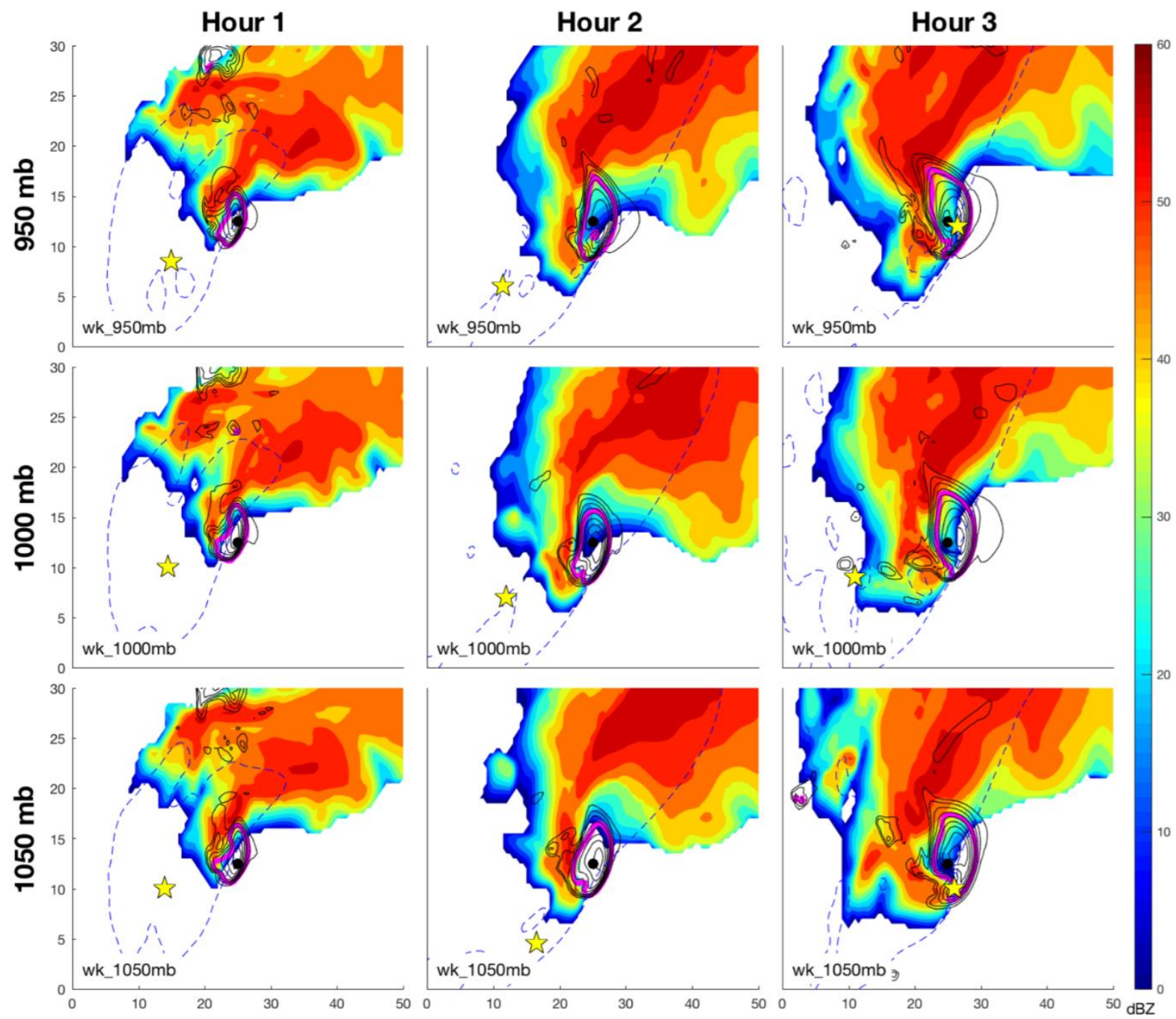

Figure 28: Hourly reflectivity fields for the storm simulations initialized with varying surface pressures of 950,1000 , and $1050 \mathrm{mb}$

Figure 29 shows the circulation field corresponding to the same times in Figure 28, including the same vertical velocity, helicity, and theta perturbation contours, as well as stormrelative wind vectors and the two circulation maxima used to compute mesocyclone separation distance. 

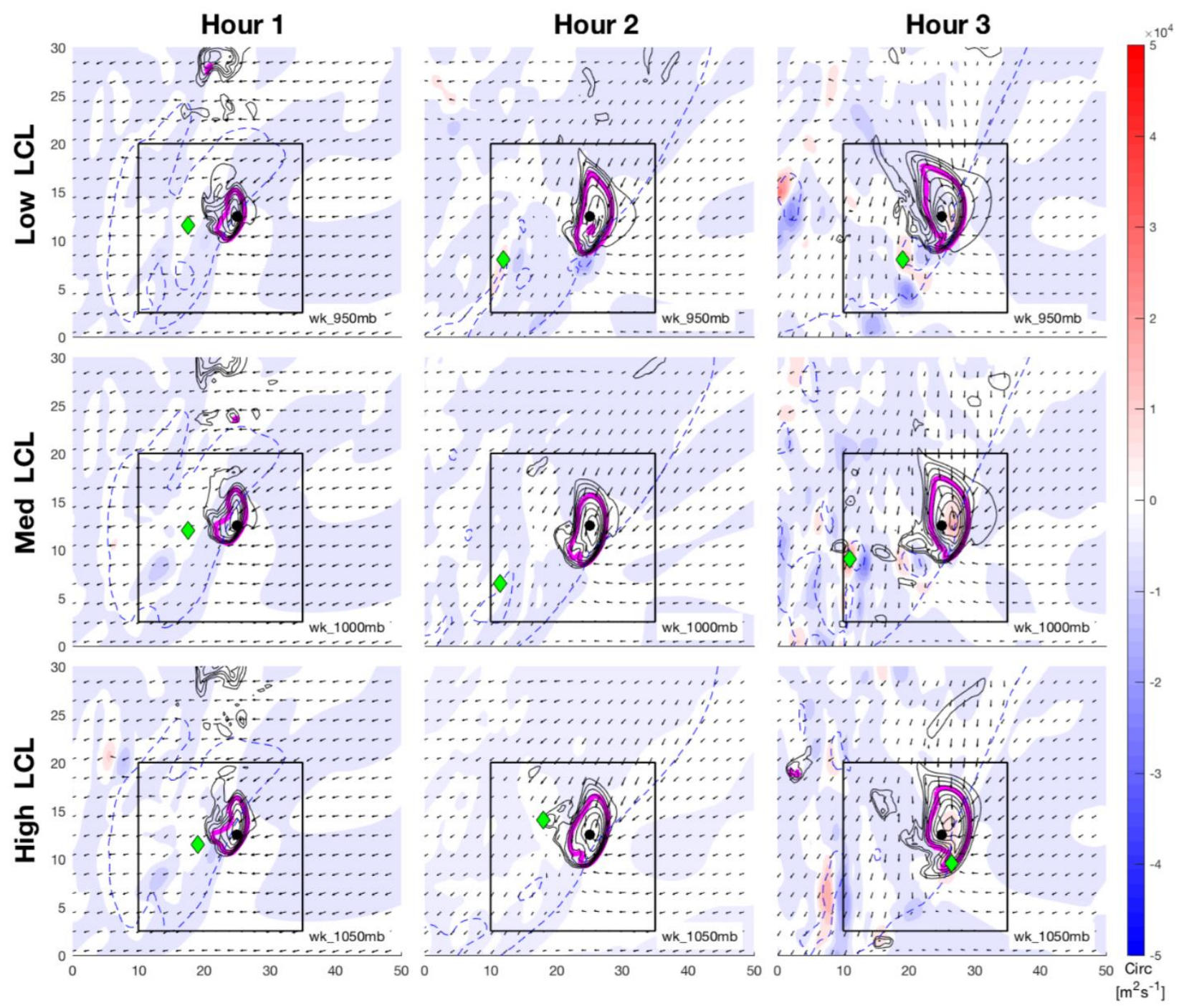

Figure 29: Hourly circulation fields for the storm simulations initialized with varying surface pressures of 950, 1000, and $1050 \mathrm{mb}$

After one hour of model integration time, the near-surface circulation field for each run contains a weakly positive (cyclonic) circulation, though this circulation is constrained behind the midlevel mesocyclone. At Hour 2, this weak circulation has shifted even farther westward in both the 950 and 1000-mb simulations, while the 1050-mb simulation shows negligible circulation across most of the storm domain. By the end of the model run, all three of the simulations have developed positive circulation beneath the mid-level mesocyclone, though the exact placement of 
the low-level circulation maximum varies due to additional circulation couplets that have formed in the vicinity of the rear-flank downdraft. In order to verify that these slight differences are not an indication of systematic differences between the various runs, time series of pertinent variables during the final two hours of integration in each model run are shown in Figure 30, including mesocyclone separation distance, maximum mid-level (4 km AGL) vertical velocity, circulation fraction (as defined in Figure 21).

Despite generally small differences in timing, these time series confirm the observations gathered from the presented reflectivity and circulation fields that the three simulations develop comparable storms that evolve in a similar manner. Each experiment displays a gradual increase in vertical vorticity, updraft helicity and strength, circulation and overlap of surface and lowlevel circulation with time, all of which reach their maxima near the end of model integration and coincide with an abrupt drop in mesocyclone separation distance. There are several time steps at which the time series diverge, such as the 1000 -mb maximum vertical vorticity at $7800 \mathrm{~s}$, but further analysis reveals that these anomalous jumps correspond to circulations on the outer periphery of the inner domain in which the maxima are computed, and therefore do not represent a distinct difference in the dynamically relevant portions of the storms. The sensitivity of variables (particularly mesocyclone separation distance) to the prescribed analysis window and the existence of multiple circulations with similar magnitudes within said window will be taken into consideration during later analysis. That said, these sensitivity results indicate that, all else held constant, changing the surface pressure does not have a major impact on storm structure and evolution. Therefore our LCL alteration method can be implemented without adverse effects due to surface pressure variations, with the expectation that changes in the simulations will be due to the changes to the low-level humidity (e.g., LCL) rather than surface pressure. 

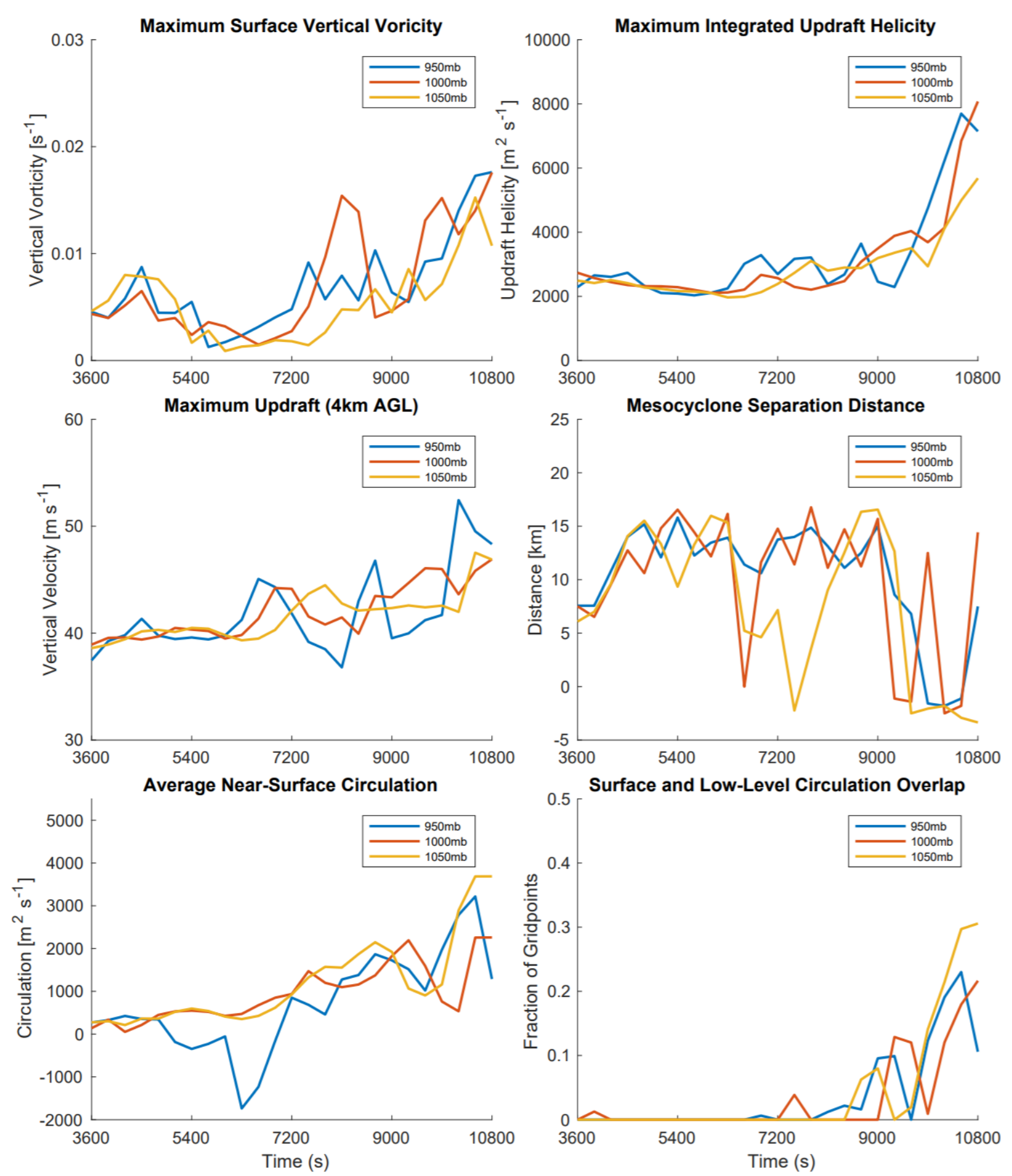

Figure 30: Time series comparison of maximum near-surface vertical vorticity, maximum integrated updraft helicity, maximum updraft (at $4 \mathrm{~km} \mathrm{AGL),} \mathrm{mesocyclone} \mathrm{separation} \mathrm{distance,} \mathrm{average} \mathrm{near-surface}$ 
circulation within the inner window defined earlier, and fraction of gridpoints with near-surface circulation exceeding $5,000 \mathrm{~m}^{2} / \mathrm{s}$ beneath the low-level mesocyclone (i.e., the area at $\mathrm{z}=1 \mathrm{~km}$ where vertical velocity is greater than $1 \mathrm{~m} \mathrm{~s}^{-1}$ and circulation exceeds $10,000 \mathrm{~m}^{2} \mathrm{~s}^{-1}$ ) for the three surface pressure simulations from Figure 28.

\section{Cold Pool/Outflow Results}

The time evolution of the cold pool simulations for the $\alpha=0^{\circ}$ and $\alpha=90^{\circ}$ wind profiles are displayed in Figures 31 and 32, respectively, including the cold pool itself as well as winds relative to the propagation of the cold pool. As per Figure 8, potential temperature perturbation is contoured beginning at a value of $-1 \mathrm{~K}$, which is chosen to represent the general size and shape of the negatively buoyant outflow. Each simulation was run over a depth of $10 \mathrm{~km}$, but only the lowest $5 \mathrm{~km}$ are shown. The presented output is from an hour into model integration, at which the cold pool has reached a relatively steady state in regards to its depth and propagation speed.

Analyzing the $\alpha=0^{\circ}$ cold pool simulations, it is clear that the three simulations are nearly identical, each displaying a relatively shallow cold pool approximately $0.5 \mathrm{~km}$ deep at its leading edge. Figure 32 shows the same homogeneity amongst the $\alpha=90^{\circ}$ simulations, each with a deeper cold pool about $2 \mathrm{~km}$ in depth at its leading edge. These differences in cold pool depth are consistent with those presented in Figure 8, with the $\alpha=0^{\circ}$ (easterly shear case in Figure 8) case corresponding to a more shallow, faster propagating density current than the $\alpha=90^{\circ}$ case (akin to the no shear case in Figure 8). These similarities indicate that the results are physically plausible even in the presence of different surface pressures. Furthermore, the similarity of the cold pools in each set of runs confirms that changing surface pressure within the storm domain does not affect the depth or propagation of its resultant outflow. These findings, along with those from the 3-D simulations in the previous section, imply that variability in cold pool properties in 
later simulations can be attributed to other environmental variables, such as LCL height and lowlevel shear orientation, rather than surface pressure.
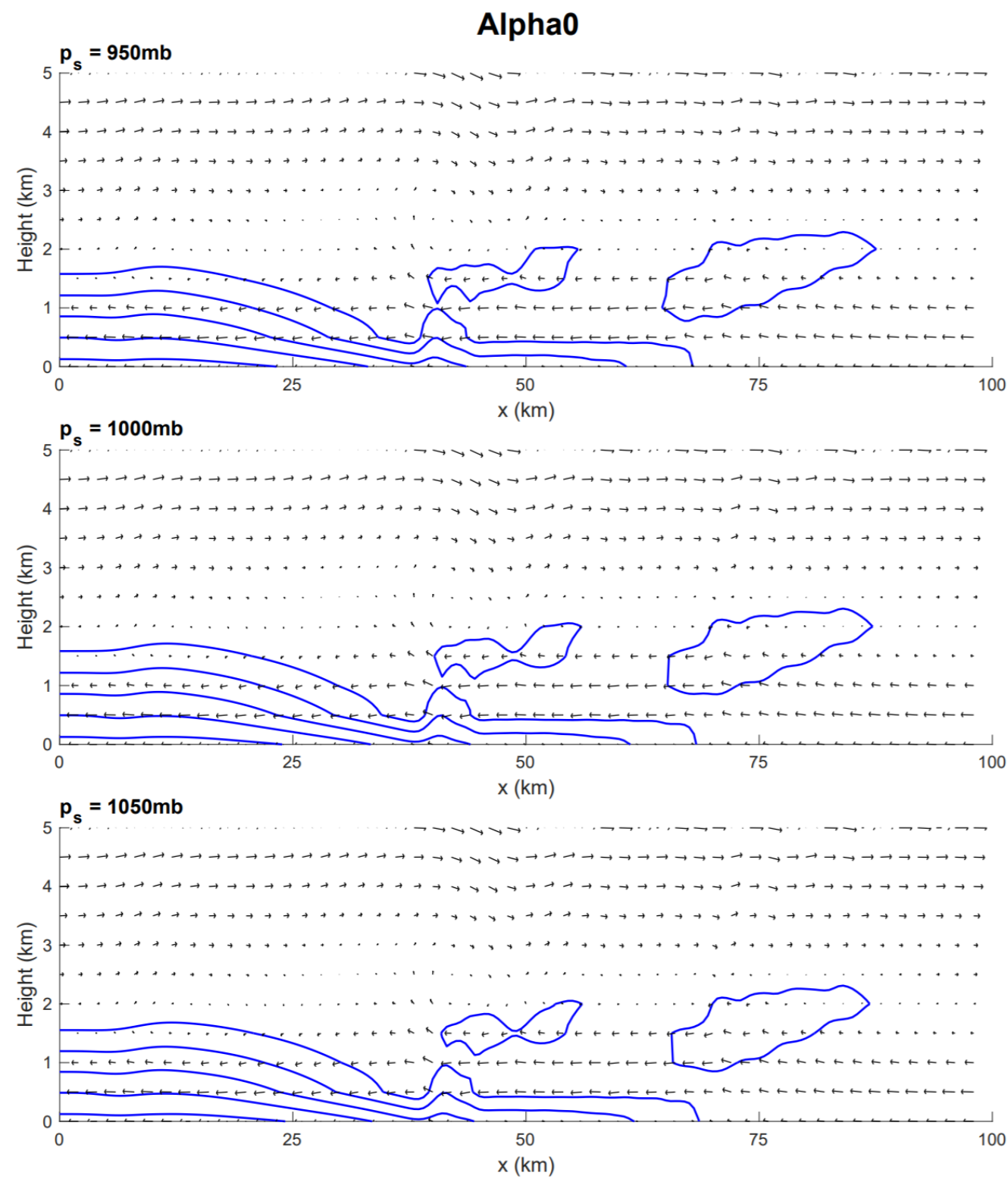

Figure 31: 2-D numerical simulations of a eastward-propagating cold pool with the $\alpha=0^{\circ}$ low-level shear profile and varying surface pressures $\left(p_{s}\right)$ of 950,1000 , and $1050 \mathrm{mb}$, shown at $3600 \mathrm{~s}$ into the simulation. The outline of the cold pool is marked in blue by negative theta perturbations contoured at 1 
$\mathrm{K}$ intervals (starting with $-1 \mathrm{~K}$ ); the wind vectors plotted are relative to the propagation speed of the cold $\operatorname{pool}(\mathrm{Uc} \approx 15 \mathrm{~m} / \mathrm{s})$

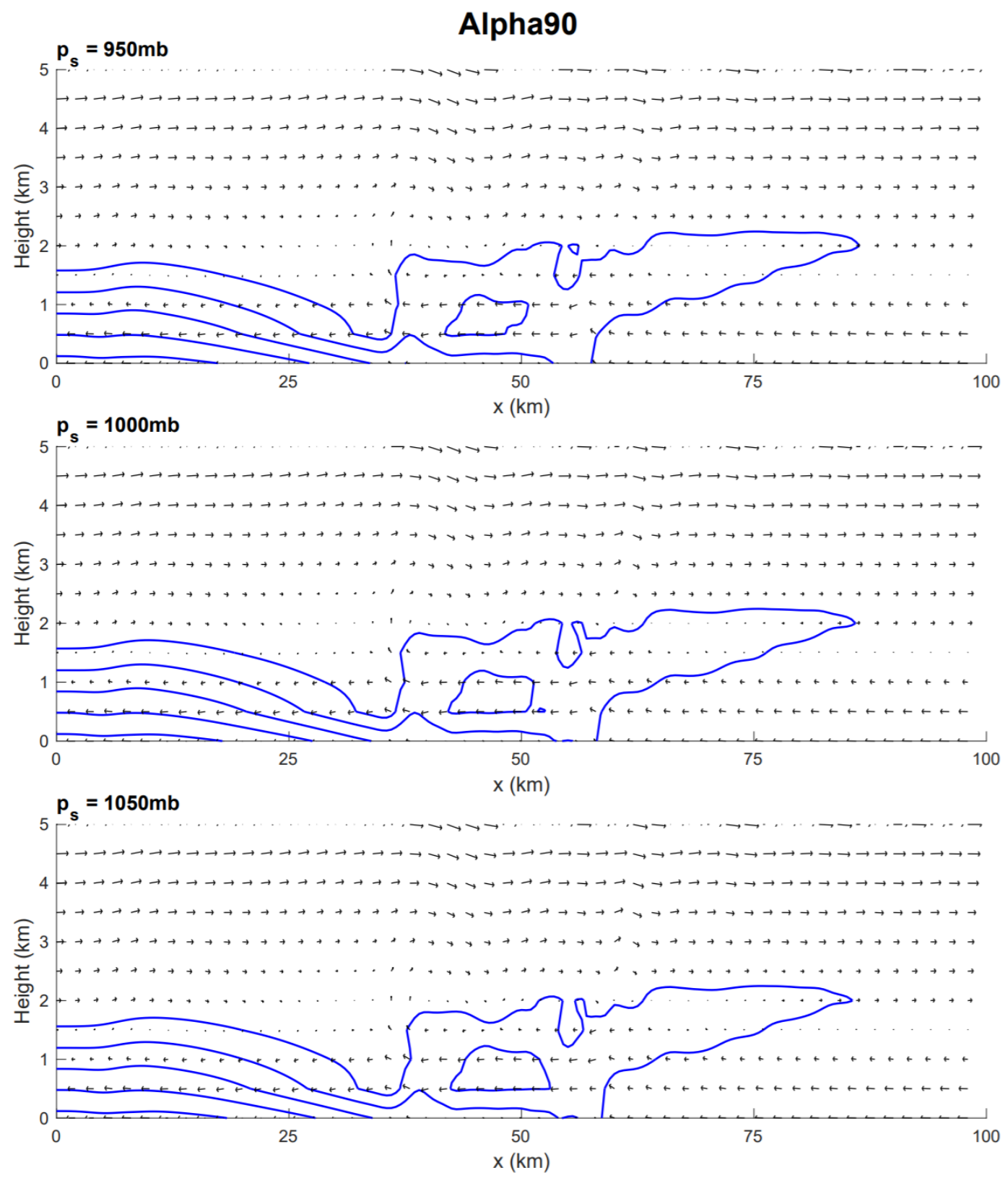

Figure 32: Same as Figure 31, but for the $\alpha=90^{\circ}$ case (with $\mathrm{Uc} \approx 12.5 \mathrm{~m} \mathrm{~s}^{-1}$ ) 